\title{
Temporal evolution of the degree distribution of alters in growing networks
}

\author{
BABAK FOTOUHI \\ Program for Evolutionary Dynamics, Harvard University, Cambridge, MA, USA \\ and \\ Institute for Quantitative Social Sciences, Harvard University, Cambridge, MA, USA \\ (e-mail: babak_fotouhi@fas.harvard.edu) \\ MICHAEL RABBAT \\ Department of Electrical and Computer Engineering, \\ McGill University, Montréal, QC, H3A OE9, Canada \\ (e-mail: michael.rabbat@mcgill.ca)
}

\begin{abstract}
The degree distribution of the neighbors of nodes in a network is a theoretically important tool that is invoked in diverse studies in network science, such as epidemics, network resilience, network search and observability, network synchronization, random walks, opinion dynamics, and other dynamical systems on networks. Many real networks grow, and their properties pertaining to the said phenomena evolve. There is a paucity of theoretical research on how the evolution of these properties depend upon time and upon the structure of the initial network. This paper addresses this problem by providing the first theoretical study of the temporal evolution of the nearest-neighbor degree distribution for arbitrary networks (with any size) in arbitrary times. The posited results enable the analysis of the structural properties of growing networks in the short-time and intermediary time regimes, which are typically ignored in favor of the steady state. We corroborate the solutions via Monte Carlo simulations on various topologies. As a byproduct of the obtained solutions, we also demonstrate that the existing result in the literature on the asymptotic behavior of the Pearson coefficient of growing networks under the preferential attachment mechanism is incorrect, and we present the correct solution.
\end{abstract}

Keywords: growing networks, degree correlation, Pearson coefficient, preferential attachment

\section{Introduction}

Dynamism of structure is one of the central features of real networks that many studies in network science seek to accommodate. Two main strands of research exist in the literature, focusing mainly on two distinct time regimes of structural change. One strand focuses on temporal fluctuations of links, such as the bursty nature of social interactions. This is the focus of the recent wave of literature on temporal networks (Holme and Saramäki, 2012). The other approach focuses on the longer time scales that characterize the growth of networks. Growth is ubiquitous across a broad array of networks. Examples include the growth of scientific citation networks (Price, 1976), network of collaborations between actors (Albert and Barabási, 2000) or scientists (Newman, 2004), ecological and biological networks (Bersini et al., 
2006; Bhan et al., 2002; Chung et al., 2003), online social networks (Kumar et al., 2010), offline social networks (Jin et al., 2001), the Internet (Faloutsos et al., 1999) and the worldwide web (Broder et al., 2000), criminal networks (Marshak et al., 2016), and the growth of the power grid due to expansion of urban areas (Pagani and Aiello, 2014). The present paper undertakes the latter approach to structural dynamics.

The degree distribution of a network - which we denote by $p_{k}$-is the probability that a uniformly chosen node (ego) is attached to $k$ other nodes. The nearestneighbor degree distribution (NNDD) - denoted by $p(\ell \mid k)$ - is the degree distribution of the neighbors (alters) of a degree- $k$ node chosen uniformly among all degree- $k$ nodes in the network. In other words, if we randomly select an ego and observe that its degree is $k$, then the probability that a randomly selected alter will have degree $\ell$ is given by $p(\ell \mid k)$. The NNDD is theoretically important and is invoked in diverse studies in network science, including network resilience (Vázquez and Moreno, 2003; Goltsev et al., 2008), epidemics on simple (Barrat et al., 2008; Boguná et al., 2003) and metapopulation (Colizza and Vespignani, 2008; Apolloni et al., 2014) networks, random walks and diffusive dynamics on networks (Barrat et al., 2008; Baronchelli et al., 2008; Baronchelli and Pastor-Satorras, 2010), transportation networks (Peruani, 2009), loop statistics in networks (Noh, 2008), contact processes on networks (Ferreira et al., 2011), network search (Fortunato et al., 2008, 2007) and observability (Hasegawa et al., 2013), evolutionary games (Zhang et al., 2015), voter model (Pugliese and Castellano, 2009; Vazquez and Eguíluz, 2008), structural cutoffs (Boguná et al., 2004a), and finding the largest eigenvalue of the adjacency matrix of the network (Restrepo et al., 2007) — which itself is invoked in numerous applications, such as epidemics (Van Mieghem, 2012; Prakash et al., 2010; Lee et al., 2013), agent-based network consensus problems (Olfati-Saber et al., 2007), and network synchronization (Restrepo et al., 2006, 2005). In all of the analyses mentioned above, the NNDD is invoked as a measure of network connectivity at the nearest-neighbor level.

The present paper focuses on the temporal evolution of the NNDD, which enables the theoretical study of how the properties of network pertaining to all the said phenomena evolve in time. More specifically, this paper answers the following question: if an arbitrary network with given degree distribution and given NNDD is subject to growth, how does its NNDD evolve in time? We seek a solution for arbitrary times that also captures the effect of the initial network.

Although there exist ample theoretical studies and results on the structural properties of growing networks, most of them are mainly restricted to the steady state (also known as the equilibrium state, the thermodynamic limit, or the $t \rightarrow \infty$ limit). ${ }^{1}$ However, no real network has infinite size. Real networks evolve over time, and this motivates studying the time evolution of the structural attributes of networks for arbitrary times. Given the observed structure of a network at a given reference time, one may wish to forecast how its structure will evolve over a period of a

1 When time is taken into account, it is again conventionally restricted to the asymptotic limit. An example is the work of Barrat and Pastor-Satorras (2005) that studies the average nearest-neighbor degree taking into account the leading term in the asymptotic limit. The effect of initial conditions has already vanished in this time regime. 
few days or weeks. This period is small compared to the typical age of massive real networks. (For example, consider a citation network; the number of papers published in a week or a month is typically much smaller than the size of the entire corpus of previous literature.) This illuminates the need for the theoretical analysis of short-time evolution of networks, for which there is little existing work.

This work is also motivated by the following observation. Due to the finite size of observed networks, existing asymptotic theoretical predictions in the literature cannot be tested. For example, failure to fit a power-law degree distribution to observed data does not imply that the corresponding network is not evolving according to a preferential attachment model, since the network may not have reached the steady-state regime yet. This paper takes a step toward addressing this problem.

To study the temporal evolution of the NNDD, we employ the most basic growth mechanism posited in the literature: preferential attachment. It is a simple generative model proposed originally by Price (1976) to model citation networks. It was more recently revived by Barabási and Albert (1999) to model the formation of scale-free (SF) networks - networks with power-law degree distributions. Until very recently (Fotouhi and Rabbat, 2013a), there has not been any theoretical analysis providing expressions for the NNDD of networks constructed by the preferential attachment mechanism. Rather, previous work has resorted to a combination of numerical simulations and the so-called "uncorrelated approximation", assuming that the degree distributions of neighboring nodes are independent. ${ }^{2}$

The first contribution of this paper is to derive an expression for the evolution of the expected NNDD of growing networks as a function of time. We assume that the network begins from an arbitrary initial condition with given degree distribution and NNDD, and that the network structure evolves according to the preferential attachment model. We corroborate the results via Monte Carlo simulations on various network topologies. We then use the obtained results and find the leading behavior of the NNDD for long times. Using the results, we demonstrate that the Pearson correlation coefficient for the preferential attachment model tends to zero for infinite system size, which is consistent with the findings in Dorogovtsev et al. (2010). We also utilize the results to study the asymptotic behavior of the Pearson correlation coefficient. Denoting the number of nodes by $N$ and the Pearson coefficient by $r$, we demonstrate that $r \rightarrow 0$ as $\log ^{2} N / \sqrt{N}$ as $N$ becomes large. This is in contrast to the result presented in Newman (2002), in which the denominator reads $N$, rather than $\sqrt{N}$. We support our finding both theoretically and via simulations.

\section{Notation and method}

Denote by $N(t)$ the number of nodes in the network at time $t$, and let $N_{k}(t)$ denote the expected number of nodes with degree $k$ at time $t$. Let $V(t)$ denote the set of nodes in the network at time $t$, and let $L(t)$ denote the number of links in the network at time $t$. For all time-dependent quantities, we take the initial conditions at time $t=0$ to be given (e.g., the initial set of nodes $V(0)$ and initial number of

\footnotetext{
2 See Fotouhi and Rabbat (2013a) and Equation (15), for a detailed list of previous work using the uncorrelated approximation.
} 


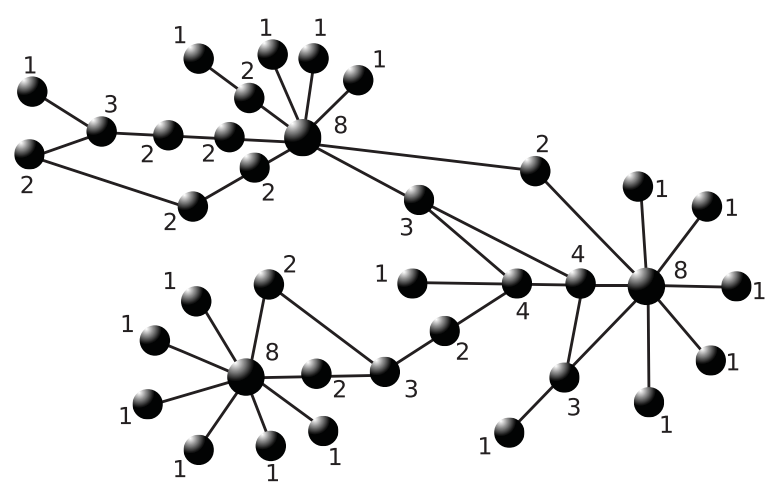

Fig. 1. An example graph used to explain the steps undertaken to find $p(\ell \mid k)$ using Equation (1). The degree of each node is written beside it.

links $L(0)$ ), and we omit the notational dependence on time when it is clear from the context. Hence, we denote the expected degree distribution by $p(k)=N_{k} / N$, where it is implied that this quantity is also time-dependent.

The growth mechanism is as follows. At each timestep, $\alpha$ new nodes are added to the network and each of them attaches to $\beta$ existing nodes. Hence, at time $t$, there are $N(0)+\alpha t$ nodes and $L(0)+\alpha \beta t$ links in the network. The new node at time $t$ is called the child of the exiting nodes to which it attaches, and the nodes to which it attaches are called its parents. The probability of attaching to a potential parent node is proportional to that node's current degree. A $(k, \ell)$-parent-child pair is a child of degree $\ell$ with a parent of degree $k$. Let $N_{k \ell}$ and $N_{\ell k}$ denote the expected number of $(k, \ell)$ - and $(\ell, k)$-parent-child pairs, respectively. Let $L_{k \ell}$ denote the expected number of links that connect a pair of nodes with degrees $k$ and $\ell$, so that $L_{k \ell}=L_{\ell k}=N_{k \ell}+N_{\ell k}$.

To calculate the NNDD for a node of degree $k$, which we denote by $p(\ell \mid k)$, we proceed as follows. First, find all the nodes with degree $k$, and then denote the collection of their neighbors by $V_{k}$. If a node appears as the neighbor of multiple degree- $k$ nodes, then it appears in $V_{k}$ with the same multiplicity. The number of elements of $V_{k}$ equals $k N_{k}$, because there are $N_{k}$ degree- $k$ nodes and each of them has $k$ neighbors. Next we count the number of nodes in $V_{k}$ with degree $\ell$, which we denote by $L_{k \ell}$. Then, the NNDD is given by

$$
p(\ell \mid k)=\frac{L_{k \ell}}{k N_{k}}=\frac{N_{k \ell}+N_{\ell k}}{k N_{k}}
$$

Example: Consider the graph depicted in Figure 1. Suppose we want to find $p(1 \mid 4)$, i.e., if we know some node has degree 4, what is the probability that a randomly chosen neighbor will have degree 1 ? There are two nodes with degree 4 in the graph. The one on the right has neighbors with degrees $8,3,4,3$, and the other one has neighbors with degrees $4,3,2,1$. Only one occurrence of degree 1 exists in $V_{4}$, so $p(1 \mid 4)=\frac{1}{8}$.

Now let us focus on $p(2 \mid 8)$ for the graph in Figure 1. This time, there are three nodes with degree 8 . The degree- 8 node on the bottom-left has neighbors with 
degrees $1,1,1,1,1,1,2,2$. The degree- 8 node on the top of the figure has neighbors with degrees $1,1,1,2,2,2,2,3$. Note that one of the degree- 2 neighbors of this node is also adjacent to the degree- 8 node on the right side. This common degree- 2 neighbor must be counted for both of the degree- 8 nodes that share it, so it will appear twice in $V_{8}$. The degree-8 node on the right-hand side of the figure has neighbors with degrees $1,1,1,1,1,2,3,4$. So, there are a total of 7 nodes who have degree 2 appearing in $V_{8}$. There is a total of $3 \times 8=24$ nodes in $V_{8}$, so $p(2 \mid 8)=\frac{7}{24}$.

To find the NNDD, we undertake the following steps. First, we determine $N_{k}(t)$ as a function of time in Section 3. Then, in Section 4, we study $N_{k \ell}$ using the master equation approach (as in Krapivsky and Redner (2001)) and find an equation that describes its time evolution. Then we solve this equation to find $N_{k \ell}$ as a function of time. Equipped with $N_{k \ell}(t)$ and $N_{k}(t)$, the NNDD follows from Equation (1).

Notational conventions: The equations mentioned above will often involve summations. To simplify the presentation, especially in expressions involving sums over multiple variables, we suppress the bounds of the summation when the indicated variable ranges from $-\infty$ to $+\infty$. Also, let $B_{b}^{a}$ denote the binomial coefficient $\left(\begin{array}{l}a \\ b\end{array}\right)$ (i.e., the number of ways to choose $b$ elements without repetition from a collection of $a$ elements). We adopt the convention that $B_{b}^{a}=0$ when $b>a$ and when either $a$ or $b$ is negative.

\section{Rate equation for $N_{k}$}

In the preferential attachment model, nodes are added to the network successively, each connecting to $\beta$ existing nodes. The attachment probabilities are proportional to the degrees of the existing nodes. Consider a node whose degree at time $t$ is $k$. When a new node is born at time $t$, the degree- $k$ node receives a link from that node with probability $\frac{k}{\sum_{k} k N_{k}(t)}$. The denominator is the sum of the degrees of all nodes at time $t$, which is equal to two times the number of links in the network at time $t$, that is, $2[L(0)+\alpha \beta t]$. Denote the average degree of the initial network by $\bar{k}(0)$. Then, we have $2 L(0)=N(0) \bar{k}(0)$. Hereafter, the quantity $2 L(0)$ will be denoted by $\lambda$. So, the probability of receiving a link for a node of degree $k$ becomes $\frac{k}{\lambda+2 \alpha \beta t}$. Each new node arrives after $\Delta t=\frac{1}{\alpha}$ time units. The following rate equation holds:

$$
N_{k}(t+\Delta t)-N_{k}(t)=\frac{\beta(k-1) N_{k-1}(t)}{\lambda+2 \alpha \beta t}-\frac{\beta k N_{k}(t)}{\lambda+2 \alpha \beta t}+\delta_{k, \beta}
$$

The first term on the right-hand side is the expected gain in the population of degree- $k$ nodes when the newly born node attaches to a node of degree $k-1$. The second term accounts for the event that a degree- $k$ node receives a link; hence, it degree increments and is no longer $k$. The last term, expressed using the delta function, simply states that each new node adds one to the population of nodes with degree $\beta$. Using the relationship $\Delta t=\frac{1}{\alpha}$, we can write Equation (2) equivalently as follows:

$$
\frac{N_{k}(t+\Delta t)-N_{k}(t)}{\Delta t}=\frac{\alpha \beta(k-1) N_{k-1}(t)}{\lambda+2 \alpha \beta t}-\frac{\alpha \beta k N_{k}(t)}{\lambda+2 \alpha \beta t}+\alpha \delta_{k, \beta}
$$


Now, we use the following time-continuous approximation to obtain:

$$
\frac{\partial N_{k}}{\partial t}=\frac{\alpha \beta(k-1) N_{k-1}}{\lambda+2 \alpha \beta t}-\frac{\alpha \beta k N_{k}}{\lambda+2 \alpha \beta t}+\alpha \delta_{k, \beta} .
$$

The error of this approximation is of order $\Delta t=\frac{1}{\alpha}$.

As we will see later in the simulations, the time-continuous approximation works surprisingly accurately even for the case of $\alpha=1$ provided that $N(0)$ is sufficiently large; experiments suggest that $N(0) \geqslant 20$ suffices. This is a reasonable requirement, noting that typical networks are significantly larger than this.

One can easily verify that the solution to Equation (4) with given initial conditions is

$$
\begin{aligned}
N_{k}(t)= & \sum_{\ell=1}^{k} N_{\ell}(0)(1-c)^{\ell} c^{k-\ell} B_{\ell-1}^{k-1}+\frac{\left(N(0) \bar{k}_{0}+2 \alpha \beta t\right)}{\beta} \frac{\beta(\beta+1)}{k(k+1)(k+2)} u(k-\beta) \\
& -\frac{N(0) \bar{k}_{0}}{\beta} \sum_{\ell=\beta}^{k} \frac{\beta(\beta+1)}{\ell(\ell+1)(\ell+2)}(1-c)^{\ell} c^{k-\ell} B_{\ell-1}^{k-1}
\end{aligned}
$$

where $u(x)$ is the Heaviside step function (i.e., $u(x)=0$ for $x<0$, and $u(x)=1$ for $x \geqslant 0)$, and $c(t)$ is defined as

$$
c(t) \stackrel{\text { def }}{=} 1-\sqrt{\frac{\lambda}{\lambda+2 \alpha \beta t}}
$$

Note that $c(t)$ is less than 1 for all $t$ and it tends to 1 as $t \rightarrow \infty$. The reader is referred to Fotouhi and Rabbat (2013b) for the detailed derivation of this solution.

Finally, let us recall that, as shown in Bollobás et al. (2001), Dorogovtsev and Mendes (2002), and Fotouhi and Rabbat (2013b), the asymptotic degree distribution is given by

$$
p(k)=\lim _{t \rightarrow \infty} \frac{N_{k}(t)}{N(t)}=\frac{2 \beta(\beta+1)}{k(k+1)(k+2)} u(k-\beta)
$$

\section{Rate equation for $N_{k \ell}$}

In this section, we study the rate equation that describes the time evolution of $N_{k \ell}$. In order for $N_{k \ell}$ to increment, the newly added node must attach to a node with degree $k-1$ or $\ell-1$. If the new node instead attaches to a node with degree $k$ or $\ell$, then $N_{k \ell}$ will decrement. Finally, the special case of $\ell=\beta$ must be taken into account separately. Each new node has degree $\beta$ upon birth, and if it attaches to a node of degree $k-1$, then $N_{k \beta}$ will increment. The following rate equation covers all these events and gives the expected change in $N_{k \ell}(t)$ upon the addition of a new node:

$$
\begin{gathered}
N_{k \ell}(t+\Delta t)-N_{k \ell}(t)=\alpha \beta \Delta t\left[\frac{(k-1) N_{k-1, \ell}(t)}{\lambda+2 \alpha \beta t}-\frac{k N_{k, \ell}(t)}{\lambda+2 \alpha \beta t}+\frac{(\ell-1) N_{k, \ell-1}(t)}{\lambda+2 \alpha \beta t}\right. \\
\left.-\frac{\ell N_{k, \ell}(t)}{\lambda+2 \alpha \beta t}+\frac{(k-1) N_{k-1}(t)}{\lambda+2 \alpha \beta t} \delta(\ell, \beta)\right]
\end{gathered}
$$


Similar to Section 3, we employ the time-continuous approximation to obtain

$$
\begin{aligned}
\frac{\partial N_{k \ell}}{\partial t}= & \left\{\frac{(k-1) N_{k-1, \ell}}{\lambda+2 \alpha \beta t}-\frac{k N_{k, \ell}}{\lambda+2 \alpha \beta t}+\frac{(\ell-1) N_{k, \ell-1}}{\lambda+2 \alpha \beta t}-\frac{\ell N_{k, \ell}}{\lambda+2 \alpha \beta t}\right. \\
& \left.+\frac{(k-1) N_{k-1}}{\lambda+2 \alpha \beta t} \delta(\ell, \beta)\right\} \alpha \beta
\end{aligned}
$$

This is a differential equation in the time domain, and a difference equation in both $k$ and $\ell$. We use the generating function approach (analogous to the so-called Z-transform) to proceed. Let us define the generating function

$$
\psi(z, y, t)=\sum_{k, \ell} N_{k, \ell} z^{-k} y^{-\ell}
$$

We denote the generating function for $N_{k}(t)$ by $G(\cdot)$. That is,

$$
G(z, t) \stackrel{\text { def }}{=} \sum_{k} N_{k}(t) z^{-k}
$$

Given the generating function $\psi(z, y, t)$ for the sequence $N_{k, \ell}(t)$, it is straightforward to show that the generating function for $k N_{k, \ell}$ is $-z \frac{\partial \psi(z, y, t)}{\partial z}$. Also, the generating function for $N_{k-1, \ell}$ is $z^{-1} \psi(z, y, t)$. Similarly, the generating function for $\ell N_{k, \ell}$ is $-y \frac{\partial \psi(z, y, t)}{\partial y}$ and the generating function for $N_{k, \ell-1}$ is $y^{-1} \psi(z, y, t)$. Using these properties, from Equation (10), we obtain

$$
\begin{aligned}
\frac{\partial}{\partial t} \psi(z, y, t)= & \frac{\alpha \beta(z-1)}{(\lambda+2 \alpha \beta t)} \frac{\partial}{\partial z} \psi(z, y, t) \\
& +\frac{\alpha \beta(y-1)}{(\lambda+2 \alpha \beta t)} \frac{\partial}{\partial y} \psi(z, y, t)-\frac{\alpha \beta y^{-\beta}}{\lambda+2 \alpha \beta t} \frac{\partial G(z, t)}{\partial z}
\end{aligned}
$$

For brevity in the following, let us define

$$
K(z, y, t) \stackrel{\text { def }}{=}-\frac{\alpha \beta y^{-\beta}}{\lambda+2 \alpha \beta t} \frac{\partial G(z, t)}{\partial z}
$$

An explicit expression for $K(z, y, t)$ is obtained in Appendix A. We can rewrite Equation (13) as follows:

$$
\frac{\partial}{\partial t} \psi(z, y, t)=K(z, y, t)+\frac{\alpha \beta(z-1)}{(\lambda+2 \alpha \beta t)} \frac{\partial}{\partial z} \psi(z, y, t)+\frac{\alpha \beta(y-1)}{(\lambda+2 \alpha \beta t)} \frac{\partial}{\partial y} \psi(z, y, t)
$$

Rearranging the terms in Equation (15), the differential equation we need to solve becomes

$$
\frac{\partial}{\partial t} \psi(z, y, t)-\frac{\alpha \beta(z-1)}{(\lambda+2 \alpha \beta t)} \frac{\partial}{\partial z} \psi(z, y, t)-\frac{\alpha \beta(y-1)}{(\lambda+2 \alpha \beta t)} \frac{\partial}{\partial y} \psi(z, y, t)=K(z, y, t)
$$

We use the method of characteristics to solve this partial differential equation. ${ }^{3}$

3 The reader is referred to a textbook on linear partial differential equations, such as Zwillinger (1998, chap. 2), for detailed discussions about the method, and to Appendix D in Fotouhi and Rabbat (2013b) for a brief and quick introduction. 
Define

$$
\left\{\begin{array}{l}
C_{1} \stackrel{\text { def }}{=}(z-1)^{2}(\lambda+2 \alpha \beta t) \\
C_{2} \stackrel{\text { def }}{=} \frac{z-1}{y-1}
\end{array}\right.
$$

In Appendix B, we show that the solution to Equation (16) is

$$
\psi(z, y, t)=\frac{-C_{1}}{\alpha \beta} \int^{z} \frac{K\left[x, \frac{x-1+C_{2}}{C_{2}}, \frac{C_{1}}{2 \alpha \beta(x-1)^{2}}-\frac{\lambda}{2 \alpha \beta}\right]}{(x-1)^{3}} d x+\Phi\left(C_{1}, C_{2}\right)
$$

This expression for $\psi(\cdot)$ satisfies Equation (16) for any differentiable function $\Phi(\cdot)$, and $\Phi(\cdot)$ is uniquely determined for given initial conditions. Note that inside the integral, $C_{1}$ and $C_{2}$ are treated as constants and their dependence on time and $z$ must be ignored while performing the integration. After the integration, $C_{1}$ and $C_{2}$ are replaced as functions of $z, y$ and $t$ as given in (17).

For expository purposes, let us temporarily define

$$
\mathscr{2}(z, y, t) \stackrel{\text { def }}{=} \frac{-C_{1}}{\alpha \beta} \int^{z} \frac{K\left[x, \frac{x-1+C_{2}}{C_{2}}, \frac{C_{1}}{2 \alpha \beta(x-1)^{2}}-\frac{\lambda}{2 \alpha \beta}\right]}{(x-1)^{3}} d x
$$

so that we can equivalently express Equation (18) as

$$
\psi(z, y, t)=\mathscr{2}(z, y, t)+\Phi\left(C_{1}, C_{2}\right)
$$

Taking the inverse transform of this expression yields $N_{k \ell}(t)$. This inversion is carried out in Appendix C. To this end, we first find the unknown function $\Phi(\cdot, \cdot)$ by examining at Equation (20) at the outset, that is, at $t=0$, and using the initial conditions $N_{k \ell}(0)$. Denoting the inverse transform of $\mathscr{2}(z, y, t)$ by $Q_{k \ell}(t)$, and using the definition in Equation (6) for brevity, the result is

$$
\begin{aligned}
N_{k \ell}(t)= & Q_{k \ell}(t)+\sum_{r, s} N_{r s}(0) B_{r-1}^{k-1} B_{s-1}^{\ell-1}\left(\frac{1-c}{c}\right)^{r+s} c^{k+\ell} \\
& -\sum_{r, s} Q_{r s}(0) B_{r-1}^{k-1} B_{s-1}^{\ell-1}\left(\frac{1-c}{c}\right)^{r+s} c^{k+\ell}
\end{aligned}
$$

Note that the bounds of summation are automatically imposed by the binomial coefficients. The terms of both sums vanish for all $r>k, s>\ell$ and for negative values of $r$ and $s$.

For a complete solution, we need the explicit form of $Q_{k \ell}(t)$ to insert in the last sum on the right-hand side of Equation (21). In Appendix D, we simplify $\mathscr{2}(z, y, t)$, 
arriving at the expression

$$
\begin{aligned}
& \mathscr{2}(z, y, t)=\frac{-2(\lambda+2 \alpha \beta t)}{\beta} \\
& \quad \times \sum_{s=\beta}^{\infty} \sum_{m=\beta+s+2}^{\infty} \sum_{v=\beta}^{m-s-2} \sum_{\mu=0}^{v-\beta} \frac{B_{2}^{s-\beta}}{s} \frac{B_{1}^{m-s-v-1} B_{\beta-1}^{v-1} B_{\mu}^{v-\beta}(-1)^{\mu}}{(m-1)} \frac{(z-1)^{\mu+\beta+2}}{(y-1)^{\mu+\beta}} z^{-m+1} \\
& -\sqrt{\frac{\lambda+2 \alpha \beta t}{\lambda}} G_{0}^{\prime}\left(z^{\prime}\right) \sum_{m=\beta+2}^{\infty} \sum_{v=\beta}^{m-2} \sum_{\mu=0}^{v-\beta} \frac{B_{1}^{m-v-1} B_{\beta-1}^{v-1} B_{\mu}^{v-\beta}(-1)^{\mu}}{(m-1)} \frac{(z-1)^{\mu+\beta+1}}{(y-1)^{\mu+\beta}} z^{-m+1} \\
& -\frac{2(\lambda+2 \alpha \beta t)}{\beta} F\left(z^{\prime}\right) \sum_{m=\beta+2}^{\infty} \sum_{v=\beta}^{m-2} \sum_{\mu=0}^{v-\beta} \frac{B_{1}^{m-v-1} B_{\beta-1}^{v-1} B_{\mu}^{v-\beta}(-1)^{\mu}}{(m-1)} \frac{(z-1)^{\mu+\beta+2}}{(y-1)^{\mu+\beta}} z^{-m+1} \\
& +\frac{1}{\beta}(\lambda+2 \alpha \beta t) \sum_{m=2 \beta+4}^{\infty} \sum_{v=\beta}^{m-\beta-4} \sum_{\mu=0}^{v-\beta} \frac{B_{3}^{m-\beta-v-1} B_{\beta-1}^{v-1} B_{\mu}^{v-\beta}(-1)^{\mu}}{(m-1)} \frac{(z-1)^{\mu+\beta+2}}{(y-1)^{\mu+\beta}} z^{-m+1} \\
& -\frac{\lambda}{\beta}\left(\frac{\lambda}{\lambda+2 \alpha \beta t}\right)^{\frac{\beta}{2}} \sum_{m=\beta+2}^{\infty} \sum_{v=\beta}^{m-2} \sum_{\mu=0}^{v-\beta} \frac{B_{1}^{m-v-1} B_{\beta-1}^{v-1} B_{\mu}^{v-\beta}(-1)^{\mu}}{(m-1)} \frac{(z-1)^{\mu+\beta}}{(y-1)^{\mu+\beta}} \frac{z^{-m+1}}{(z-c)^{\beta}}
\end{aligned}
$$

where $z^{\prime} \stackrel{\text { def }}{=} \frac{z-c}{1-c}, G_{0}(z)$ is the generating function of the degree distribution of the initial network, and the function $F(\cdot)$ is defined as $\int^{z} \frac{x^{-\beta}}{(x-1)^{3}} d x$. For easy reference, let us also remind the reader that $\lambda$ is twice the number of links in the initial network.

Now, let us define

$$
\begin{cases}\hat{f}_{k} & \stackrel{\text { def }}{=}-\sum_{r=\beta}^{k} \frac{B_{2}^{r-\beta} B_{r-1}^{k-1}}{r}(1-c)^{r} c^{k-r} \\ \hat{g}_{k} & \stackrel{\text { def }}{=}-\sum_{r=\beta}^{k}(r-1) N_{r-1}(0)(1-c)^{r} c^{k-r} B_{r-1}^{k-1}\end{cases}
$$

In Appendix E, we take the inverse transform of Equation (22) and find that in the range $\ell<\beta$ the value of $Q_{k \ell}(t)$ is zero, and for $\ell \geqslant \beta$, we have

$$
\begin{aligned}
& Q_{k \ell}(t)=-(\lambda+2 \alpha \beta t) \\
& \times {\left[\frac{-\beta(\beta+1)}{k(k+1) \ell(\ell+1)}+\frac{(-1)^{\beta} B_{\beta-1}^{k-1}}{\ell(\ell+1)} \sum_{\mu=\beta}^{\ell}(-1)^{\mu} \frac{B_{\beta}^{\mu} B_{\mu+1}^{\ell+1}}{B_{k-\beta}^{\mu+k+2}}\right] u(k-\beta-1) } \\
&- \beta \frac{\hat{g}_{k}}{\ell(\ell+1)}\left(\sqrt{\frac{\lambda+2 \alpha \beta t}{\lambda}}\right)+\frac{2(\lambda+2 \alpha \beta t)}{\ell(\ell+1)}\left[\hat{f}_{k}-\hat{f}_{k+1}\right] \\
&-\lambda\left(\frac{\lambda}{\lambda+2 \alpha \beta t}\right)^{\frac{\beta}{2}} \frac{1}{\ell(\ell+1)} \sum_{j=\beta}^{k-1} c^{j-\beta} B_{\beta-1}^{j-1}
\end{aligned}
$$

The value of $Q_{r s}(0)$ is also obtained in Appendix E. We have to insert this into Equation (21) to obtain $N_{k \ell}$. However, it is $N_{k \ell}+N_{\ell k}$ that appears as the numerator in Equation (1), not $N_{k \ell}$ alone. In Appendix F, we directly calculate $N_{k \ell}+N_{\ell k}$. 
Then, after a number of simplifications, we obtain the following expression for the numerator of Equation (1):

$$
\begin{aligned}
& N_{\ell k}(t)+N_{k \ell}(t) \\
& =\left(N(0) \bar{k}_{0}+2 \alpha \beta t\right) \frac{\beta(\beta+1)}{\ell k(k+1)(k+2)}\left[\frac{k+2}{\ell+1}-\frac{B_{\beta+1}^{2 \beta+2} B_{\ell-\beta}^{k+\ell-2 \beta}}{B_{\ell}^{k+\ell+2}}\right] u(k-\beta) u(\ell-\beta) \\
& +\beta \sqrt{\frac{N(0) \bar{k}_{0}+2 \alpha \beta t}{N(0) \bar{k}_{0}}} \sum_{r=1}^{k} \frac{(r-1) N_{r-1}(0)(1-c)^{r} c^{k-r} B_{r-1}^{k-1}}{\ell(\ell+1)} u(\ell-\beta) \\
& +\beta \sqrt{\frac{N(0) \bar{k}_{0}+2 \alpha \beta t}{N(0) \bar{k}_{0}}} \sum_{r=1}^{\ell} \frac{(r-1) N_{r-1}(0)(1-c)^{r} c^{\ell-r} B_{r-1}^{\ell-1}}{k(k+1)} u(k-\beta) \\
& +2\left(N(0) \bar{k}_{0}+2 \alpha \beta t\right) \\
& \times\left[\sum_{r=\beta}^{k+1} \frac{B_{2}^{r-\beta} B_{r-1}^{k}}{r \ell(\ell+1)}(1-c)^{r} c^{k+1-r}-\sum_{r=\beta}^{k} \frac{B_{2}^{r-\beta} B_{r-1}^{k-1}}{r \ell(\ell+1)}(1-c)^{r} c^{k-r}\right] u(\ell-\beta) \\
& +2\left(N(0) \bar{k}_{0}+2 \alpha \beta t\right) \\
& \times\left[\sum_{r=\beta}^{\ell+1} \frac{B_{2}^{r-\beta} B_{r-1}^{\ell}}{r k(k+1)}(1-c)^{r} c^{\ell+1-r}-\sum_{r=\beta}^{\ell} \frac{B_{2}^{r-\beta} B_{r-1}^{\ell-1}}{r k(k+1)}(1-c)^{r} c^{\ell-r}\right] u(k-\beta) \\
& -N(0) \bar{k}_{0}\left(\frac{N(0) \bar{k}_{0}}{N(0) \bar{k}_{0}+2 \alpha \beta t}\right)^{\frac{\beta}{2}}\left[\sum_{j=\beta}^{k-1} \frac{c^{j-\beta} B_{\beta-1}^{j-1}}{\ell(\ell+1)} u(\ell-\beta)+\sum_{j=\beta}^{\ell-1} \frac{c^{j-\beta} B_{\beta-1}^{j-1}}{k(k+1)} u(k-\beta)\right] \\
& +\sum_{r=1}^{k} \sum_{s=1}^{\ell}\left[N_{r s}(0)+N_{s r}(0)\right] B_{r-1}^{k-1} B_{s-1}^{\ell-1}\left(\frac{1-c}{c}\right)^{r+s} c^{k+\ell} \\
& -N(0) \bar{k}_{0} \beta(\beta+1) \sum_{r=\beta}^{k} \sum_{s=\beta}^{\ell} \frac{\frac{r+2}{s+1}-\frac{B_{\beta+1}^{2 \beta+2} B_{s-\beta}^{r+s-2 \beta}}{B_{s}^{r+s+2}}}{r(r+1)(r+2) s} B_{r-1}^{k-1} B_{s-1}^{\ell-1}\left(\frac{1-c}{c}\right)^{r+s} c^{k+\ell} \\
& +N(0) \bar{k}_{0} \beta(\beta+1) \sum_{r=\beta}^{k} \sum_{s=\beta}^{\ell} \frac{2-\delta_{r, \beta}-\delta_{s, \beta}}{r(r+1) s(s+1)} B_{r-1}^{k-1} B_{s-1}^{\ell-1}\left(\frac{1-c}{c}\right)^{r+s} c^{k+\ell} \\
& -\beta \sum_{r=1}^{k} \sum_{s=\beta}^{\ell} \frac{(r-1) N_{r-1}(0)}{s(s+1)} B_{r-1}^{k-1} B_{s-1}^{\ell-1}\left(\frac{1-c}{c}\right)^{r+s} c^{k+\ell} \\
& -\beta \sum_{r=\beta}^{k} \sum_{s=1}^{\ell} \frac{(s-1) N_{s-1}(0)}{r(r+1)} B_{r-1}^{k-1} B_{s-1}^{\ell-1}\left(\frac{1-c}{c}\right)^{r+s} c^{k+\ell}
\end{aligned}
$$

For the denominator of Equation (1), using Equation (5), we have

$$
\begin{aligned}
k N_{k}(t)= & k \sum_{r=1}^{k} N_{r}(0)(1-c)^{r} c^{k-r} B_{r-1}^{k-1}+\frac{\left(N(0) \bar{k}_{0}+2 \alpha \beta t\right)(\beta+1)}{(k+1)(k+2)} u(k-\beta) \\
& -\frac{N(0) \bar{k}_{0}}{\beta} \sum_{r=\beta}^{k} \frac{\beta(\beta+1)}{(r+1)(r+2)}(1-c)^{r} c^{k-r} B_{r}^{k}
\end{aligned}
$$


Dividing Equation (25) by Equation (26) yields the desired result, that is, $p(\ell \mid k)$ as a function of time. For the convenience of the reader, let us repeat the definition of $c$ here

$$
c=1-\sqrt{\frac{N(0) \bar{k}_{0}}{N(0) \bar{k}_{0}+2 \alpha \beta t}}
$$

\section{The steady state}

\subsection{The NNDD}

Dividing the result presented in Equation (25) by the one given in Equation (26) yields the expected value of the NNDD as a function of time. The result is visibly long and theoretically unwieldy. It might be plausible to use this theoretical expression in its present guise in numerical computations. To acquire an expression for the NNDD to be conveniently utilized in theoretical analysis however, simplifications appear indispensable.

Let us investigate the steady-state limit of the NNDD. Recall that $p(\ell \mid k)$ satisfies Equation (1). The numerator of $p(\ell \mid k)$ is given in Equation (25), and the denominator is given in Equation (26). First let us investigate the behavior of the denominator in the limit as $t \rightarrow \infty$. Note that in this limit, $c \rightarrow 1$. Both the first and the third sums on the right-hand side of (26) comprise terms with factors of the form $(1-c)^{r}$, with $r>0$ for all of them. Hence, these two sums vanish in the steady state, and only the second term remains. So, we have

$$
\lim _{t \rightarrow \infty} k N_{k}(t)=\frac{\left(N(0) \bar{k}_{0}+2 \alpha \beta t\right)(\beta+1)}{(k+1)(k+2)} u(k-\beta)
$$

Now, we turn our attention to the steady-state value of the numerator of $p(\ell \mid k)$, given in Equation (25). It can be easily seen that every term on the right-hand side of Equation (25) involve nonzero powers of $(1-c)$, except the first term. So, in the steady state, only the first term survives. We have

$$
\begin{aligned}
\lim _{t \rightarrow \infty} N_{\ell k}(t)+N_{k \ell}(t)= & \left(N(0) \bar{k}_{0}+2 \alpha \beta t\right) \frac{\beta(\beta+1)}{\ell k(k+1)(k+2)} \\
& \times\left[\frac{k+2}{\ell+1}-\frac{B_{\beta+1}^{2 \beta+2} B_{\ell-\beta}^{k+\ell-2 \beta}}{B_{\ell}^{k+\ell+2}}\right] u(k-\beta) u(\ell-\beta)
\end{aligned}
$$

Now, we can find the NNDD in the steady state by dividing Equation (28) by Equation (29). The $\left(N(0) \bar{k}_{0}+2 \alpha \beta t\right)$ factors that appear in both the numerator and the denominator cancel out, and the final result is

$$
\lim _{t \rightarrow \infty} p(\ell \mid k)=\frac{\beta}{\ell k}\left[\frac{k+2}{\ell+1}-\frac{B_{\beta+1}^{2 \beta+2} B_{\ell-\beta}^{k+\ell-2 \beta}}{B_{\ell}^{k+\ell+2}}\right] u(k-\beta) u(\ell-\beta)
$$

Note that the NNDD vanishes if either $k$ or $\ell$ are smaller than $\beta$. This is due to the fact that in the steady state, the effects of initial conditions can be ignored. This means that in this time regime, all nodes are those added to the network at some 
$t>0$, with an initial degree $\beta$. So, every node has at least degree $\beta$ in this time limit.

Also, note that also in the special case of $k=\ell=\beta$, the NNDD vanishes:

$$
\lim _{t \rightarrow \infty} p(\beta \mid \beta)=\frac{\beta}{\beta^{2}}\left[\frac{\beta+2}{\beta+1}-\frac{B_{\beta+1}^{2 \beta+2} B_{0}^{0}}{B_{\beta}^{2 \beta+2}}\right]=\frac{1}{\beta}\left[\frac{\beta+2}{\beta+1}-\frac{B_{\beta+1}^{2 \beta+2}}{B_{\beta}^{2 \beta+2}}\right]=0
$$

This can be intuitively interpreted as follows. In the steady state, if a node has degree $\beta$, it means that after it has been added to the network, it has received no link from subsequently-added nodes. When both $k$ and $\ell$ are equal to $\beta$, this means that we have two nodes that are adjacent and both have degree $\beta$. One of them has to be added prior to the other one. The degree of the older node was already at least $\beta$ when the younger node was born, so the degree of the older node must at least be $\beta+1$ when it receives one additional link from the younger node. So the situation where both of them have degree $\beta$ cannot happen, hence the zero probability.

Now, consider the case where $k=\beta$ and $\ell>\beta$; a certain node with given degree $k$ is considered (call it node $x$ ), and the degree distribution of its neighbors is given by $p(\ell \mid \beta)$. In this case, we can immediately say that all the neighbors of $x$ are older than $x$. This is because if any of them was younger, it would have incremented the degree of $x$ beyond $\beta$ upon attaching to $x$. Since the degree of $x$ equals its initial degree upon birth, all neighbors of $x$ must precede it. The same argument holds for the case of $\ell=\beta$ and $k>\beta$. In this case, node $x$ has degree more than $\beta$ and all of its neighbors that constitute $p(\beta \mid k)$ are added to the network after $x$. Note that this argument cannot be extended to the case where both $k$ and $\ell$ are greater than $\beta$. A node that is younger than its neighbors might have a greater degree, if both of them have degrees greater than $\beta$.

\subsection{Vanishing Pearson coefficient in the thermodynamic limit}

We demonstrate the compatibility of our result in Equation (30) with the results obtained in Dorogovtsev et al. (2010) regarding the assortativity of the BarabásiAlbert model in the steady state. It is contended in Dorogovtsev et al. (2010) that in the limit as $t \rightarrow \infty$, the Pearson correlation coefficient vanishes for the BarabásiAlbert model, regardless of the value of $\beta$. Here, we confirm this result by explicitly using the expression for the NNDD in the steady state that is given in Equation (30).

Let $p(k, \ell)$ be the fraction of links that are incident to nodes of degree $k$ and $\ell$. In accordance with Dorogovtsev et al. (2010), the Pearson coefficient is defined as follows:

$$
r \stackrel{\text { def }}{=} \frac{\left[\sum_{k \ell} k \ell p(k, \ell)\right]-\frac{\left(\overline{k^{2}}\right)^{2}}{\bar{k}^{2}}}{\frac{\overline{k^{3}}}{\bar{k}}-\frac{\left(\overline{k^{2}}\right)^{2}}{\bar{k}^{2}}}
$$

Let us denote the sum in the numerator by $\overline{k \ell}$. To calculate $\overline{k \ell}$, we need to have an explicit expression for $p(k, \ell)$. Using Equation (1), and noting that in the long time limit the total number of links in the network equals $N \beta$, we can relate the 
joint degree distribution $p(k, \ell)$ to the NNDD $p(\ell \mid k)$ as follows:

$$
p(k, \ell)=\frac{L_{k \ell}}{N \beta}=\frac{k N_{k} p(\ell \mid k)}{N \beta}=\frac{1}{\beta} k p(k) p(\ell \mid k)
$$

The degree distribution of the Barabási-Albert model in the steady state is given in Equation (7). Using the expression for $p(k)$ given in Equation (7) and the expression for $p(\ell \mid k)$ from Equation (30), we can rewrite Equation (33) as follows:

$$
p(k, \ell)=\frac{1}{\beta} k \frac{2 \beta(\beta+1)}{k(k+1)(k+2)} \frac{\beta}{\ell k}\left[\frac{k+2}{\ell+1}-\frac{B_{\beta+1}^{2 \beta+2} B_{\ell-\beta}^{k+\ell-2 \beta}}{B_{\ell}^{k+\ell+2}}\right] u(k-\beta) u(\ell-\beta)
$$

Using the properties of the binomial coefficients, we can express this equivalently as the following manifest-symmetric relation:

$$
p(k, \ell)=\frac{2 \beta(\beta+1)}{k(k+1) \ell(\ell+1)}\left[1-\frac{B_{\beta+1}^{2 \beta+2} B_{\ell-\beta}^{k+\ell-2 \beta}}{B_{\ell+1}^{k+\ell+2}}\right] u(k-\beta) u(\ell-\beta)
$$

Equipped with this expression for $p(k, \ell)$, we can calculate $\overline{k \ell}$. In the limit as $t \rightarrow \infty$, the lower bound for the possible values of the degrees is $\beta$, but there is no upper bound. We perform the calculations assuming a maximal degree value $\kappa$, and then take the limit $\kappa \rightarrow \infty$. So, when calculating every term in the numerator and the denominator of Equation (32), we only retain the leading terms because the limiting behavior is of interest.

Let us define

$$
\omega_{k, \ell} \stackrel{\text { def }}{=} \frac{B_{\beta+1}^{2 \beta+2} B_{\ell-\beta}^{k+\ell-2 \beta}}{B_{\ell+1}^{k+\ell+2}}
$$

so that we can recast Equation (35) as

$$
p(k, \ell)=\frac{2 \beta(\beta+1)}{k(k+1) \ell(\ell+1)}\left(1-\omega_{k \ell}\right) u(k-\beta) u(\ell-\beta)
$$

In Figure 2, we plot the value of $\omega_{k \ell}$ as a function of $k$ and $\ell$ for different values of $\beta$. It can be observed that in the limit of large $k$ and $\ell$, the value of $\omega_{k \ell}$ reaches a constant, which is small. The larger $\beta$ gets, the smaller the asymptotic value of $\omega_{k \ell}$ becomes. Since the asymptotic behavior of $\omega_{k \ell}$ is not close to unity, it does not alter the overall asymptotic behavior of $p(k, \ell)$. Consequently, minding only the leading terms, we can disregard $\omega_{k \ell}$ from Equation (37) and calculate $\overline{k \ell}$ as follows:

$$
\overline{k \ell}=\sum_{k=\beta}^{\kappa} \sum_{\ell=\beta}^{\kappa} k \ell \frac{2 \beta(\beta+1)}{k(k+1) \ell(\ell+1)} \sim \log \kappa \log \kappa=(\log \kappa)^{2}
$$




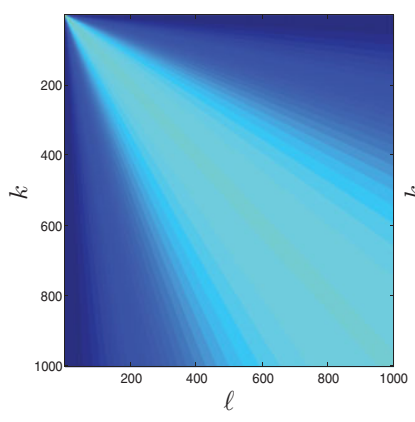

(a) $\beta=1$

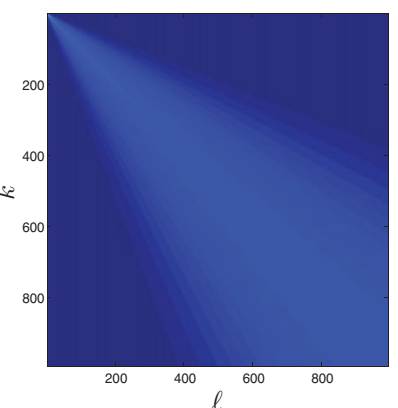

(b) $\beta=10$

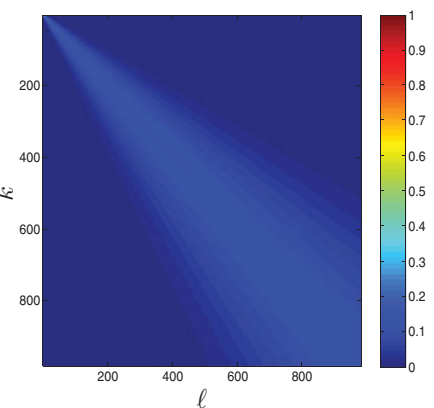

(c) $\beta=20$

Fig. 2. The value of $\omega_{k \ell}$ for three values of $\beta$. It can be seen that as $\beta$ grows, the asymptotic value of $\omega_{k \ell}$ for large values of $k$ and $\ell$ diminishes. It is the largest when $\beta=1$, which is still less than 0.5 (see the colorbar). This means that $1-\omega_{k \ell}$ is not close to zero for large values of $k$ and $\ell$, and its order of magnitude is the same as that of unity. This means that in asymptotic arguments, $1-\omega_{k \ell}$ can be replaced by unity. (a) $\beta=1$. (b) $\beta=10$. (c) $\beta=20$. (Color online)

The next term that we calculate to insert into Equation (32) is $\overline{k^{2}}$. We have

$$
\begin{aligned}
\overline{k^{2}} & =\sum_{k=\beta}^{\kappa} k^{2} \frac{2 \beta(\beta+1)}{k(k+1)(k+2)}=2 \beta(\beta+1) \sum_{k=\beta}^{\kappa} \frac{k}{(k+1)(k+2)} \\
= & 2 \beta(\beta+1) \sum_{k=\beta}^{\kappa}\left(\frac{2}{k+2}-\frac{1}{k+1}\right) \\
& \approx 2 \beta(\beta+1) \log \frac{(\kappa+2)^{2}}{\kappa+1} \approx 2 \beta(\beta+1) \log \kappa
\end{aligned}
$$

For $\overline{k^{3}}$, we have

$$
\begin{aligned}
\overline{k^{3}} & =\sum_{k=\beta}^{\kappa} k^{3} \frac{2 \beta(\beta+1)}{k(k+1)(k+2)}=2 \beta(\beta+1) \sum_{k=\beta}^{\kappa} \frac{k^{2}}{(k+1)(k+2)} \\
= & 2 \beta(\beta+1) \sum_{k=\beta}^{\kappa}\left(1+\frac{1}{k+1}-\frac{4}{k+2}\right) \\
& \approx 2 \beta(\beta+1)(\kappa+\log \kappa) \approx 2 \beta(\beta+1) \kappa .
\end{aligned}
$$

Finally, for $\bar{k}$ it suffices to note that twice the total number of links in the steady state equals $2 N \beta$, which coincides with the sum of all degrees, which is $N \bar{k}$. This readily yields $\bar{k}=2 \beta$. Inserting this expression for $\bar{k}$, together with those obtained in Equations (38)-(40) into the definition of the Pearson coefficient given in Equation (32), we get

$$
r \sim \frac{(\log \kappa)^{2}-\frac{4 \beta^{2}(\beta+1)^{2}(\log \kappa)^{2}}{4 \beta^{2}}}{\frac{2 \beta(\beta+1) \kappa}{2 \beta}-\frac{4 \beta^{2}(\beta+1)^{2}(\log \kappa)^{2}}{4 \beta^{2}}}
$$


Simplifying this, we arrive at

$$
r \sim \frac{(\log \kappa)^{2}}{\kappa}
$$

The asymptotic behavior of the Pearson coefficient, as given by Equation (42), means that in the limit of infinite network size, or equivalently, in the limit as $t \rightarrow \infty$, we have

$$
\lim _{t \rightarrow \infty} r=0
$$

This holds for all values of $\beta$. This agrees with the result obtained in Dorogovtsev et al. (2010).

\subsection{Pearson coefficient for large $N$}

To see the asymptotic behavior of the Pearson coefficient with $N$, with need to know how $\kappa$ behaves for large values of $N$. There are two conventional estimates for $\kappa$. The first one is the natural cutoff, which is defined as the degree beyond which the area under the $p(k)$ curve equals $\frac{1}{N}$. The second conventions is discussed in Boguná et al. (2004b), where it is contended that if one disallows multiple links in the model, then structural cutoff must be considered. Both the natural cutoff and the structural cutoff for the model at hand are $\kappa \sim \sqrt{N}$, as pointed out for example in Boguná et al. (2004b). Plugging this into (42), we arrive at

$$
r \sim \frac{(\log N)^{2}}{\sqrt{N}}
$$

This is inconsistent with the result presented in Newman (2002), which has the same numerator as (44), but has $N$ in the denominator rather than $\sqrt{N}$. Let us use simulations, synthesize large networks and find the Pearson coefficient as a function of $N$ to ascertain the true asymptotic behavior. If (44) is true, then $r \times \frac{\sqrt{N}}{\log ^{2} N}$ should reach a horizontal asymptote for large $N$. If the result of Newman (2002) is true, then $r \times \frac{N}{\log ^{2} N}$ should reach a horizontal asymptote for large $N$.

In Figure 3(a), we have plotted $r \times \frac{N}{\log ^{2} N}$ as a function of $N$ for a growing network. In Figure 3(b), we have plotted $r \times \frac{\sqrt{N}}{\log ^{2} N}$ as a function of $N$. We went up to $N=10^{6}$ for the network size. It is readily visible from the figures that Figure 3(b) approaches a horizontal asymptote, while 3(a) does not.

To quantify the performance of the two predictions, we use regression. The prediction in Newman (2002) states that $r$ is asymptotically a linear function of the quantity $\frac{N}{(\log N)^{2}}$. Our prediction states that $r$ is asymptotically a linear function of $\frac{\sqrt{N}}{(\log N)^{2}}$. So, we fit linear models in both cases and calculate the $R^{2}$ coefficient. For the prediction in Newman (2002), we have $R^{2}=0.923$. For our prediction, we get $R^{2}=0.998$. This confirms that $\frac{(\log N)^{2}}{\sqrt{N}}$ is the correct solution.

Finally, note that the Pearson coefficient $r(N)$ tends to zero from below as $N$ becomes large (by "from below" we mean that $r<0$, as can be seen from Figure 3). This is consistent with the results presented in Dorogovtsev et al. (2010).

It is imperative to note that vanishing Pearson coefficient does not justify the uncorrelated approximation, that is, the assertion that the NNDD can be replaced by 


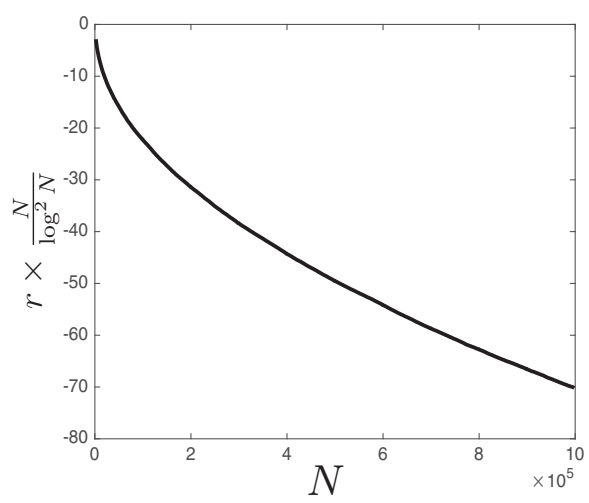

(a) The result in (Newman, 2002).

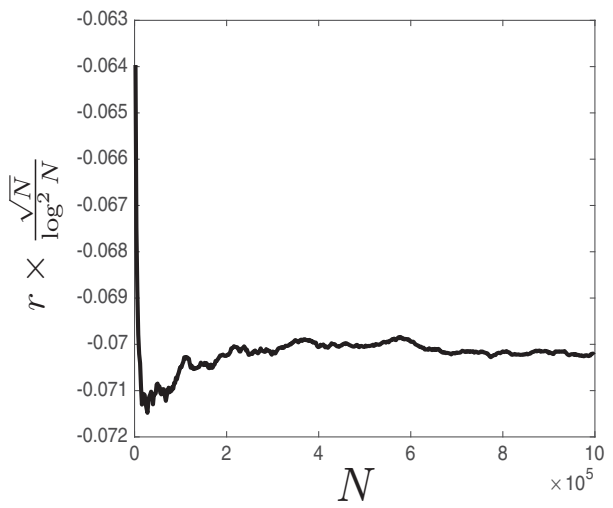

(b) The result in (44).

Fig. 3. Checking the asymptotic behavior of the Pearson correlation coefficient for large $N$. The simulations are averaged over 100 Monte Carlo trials. The minimum network size is 2,000 and the maximum network size is $10^{6}$. (a) The result in Newman (2002). (b) The result in (44).

$\frac{\left\langle k^{2}\right\rangle}{\langle k\rangle}$ (which would be obtained under the assumption that the network is locally treelike, together with the assumption that adjacent nodes are statistically independent). The fact that two networks have equal Pearson correlation coefficients (in this case, an Erdős-Rényi network and a Barabási-Albert network, both having $r=0$ in the thermodynamic limit) is not synonymous with the equality of their NNDDs. Both $\frac{\left\langle k^{2}\right\rangle}{\langle k\rangle}$ and the expression for the NNDD given in Equation (30) lead to $r=0$. Hence, from $r=0$ one cannot aptly conclude that $\frac{\left\langle k^{2}\right\rangle}{\langle k\rangle}$ is a valid expression for the NNDD of the underlying network.

\subsection{Average nearest neighbor degree}

The average nearest neighbor degree (hereinafter ANND) of nodes of degree $k$ is a function of $k$ and is defined as follows. Consider all the nodes in the network that have degree $k$. Call the set of neighbors of all of these nodes by $S_{k}$, accounting for multiplicities. That is, if a node is adjacent to, say, $b$ nodes of degree $k$, then this node should appear $b$ times in $S_{k}$. Then, we define the ANND for degree $k$ as the average degree of nodes in $S_{k}$. We denote the ANND by $k_{N N}(k)$, which can be alternatively expressed as follows:

$$
k_{N N}(k) \stackrel{\text { def }}{=} \sum_{\ell} \ell p(\ell \mid k)
$$

In Barrat and Pastor-Satorras (2005), the rate equations describing degree-degree correlations are solved in the asymptotic long-time limit, and it is shown that

$$
k_{N N}(k) \approx \frac{\beta}{2} \log N
$$

We now show that our result presented in Equation (30) can be utilized to confirm this asymptotic expression for the ANND. Let us calculate the ANND 
explicitly:

$$
\begin{aligned}
k_{N N}(k) & =\sum_{\ell=\beta}^{\kappa} \ell p(\ell \mid k)=\sum_{\ell=\beta}^{\kappa} \ell \frac{\beta}{\ell k}\left[\frac{k+2}{\ell+1}-\frac{B_{\beta+1}^{2 \beta+2} B_{\ell-\beta}^{k+\ell-2 \beta}}{B_{\ell}^{k+\ell+2}}\right] \\
& \approx \sum_{\ell=\beta}^{\kappa} \frac{\beta}{k} \frac{k+2}{\ell+1} \approx \beta\left(\frac{k+2}{k}\right) \log \kappa \approx \beta \log \kappa
\end{aligned}
$$

Where in concert with the previous section, we have used the fact that $w_{k \ell}$, defined in Equation (36), is negligible as compared to unity, in the limit of large $k$ or large $\ell$.

Note that the results in Equations (47) and (46) coincide if we have $\kappa \approx \sqrt{N}$. This is true if we assume $\kappa$ to be the natural cutoff, which is defined as the degree beyond which the area under the $p(k)$ curve equals $\frac{1}{N}$. The calculation is straightforward:

$$
\int_{\kappa}^{\infty} k^{-3} d k \sim \frac{1}{N} \Longrightarrow \kappa^{-2} \sim \frac{1}{N} \Longrightarrow \kappa \sim \sqrt{N}
$$

In Boguná et al. (2004b), it is contended that if one disallows multiple links in the model, then in addition to the natural cutoff, structural cutoff must be considered. For the model considered here, the asymptotic degree distribution follows a power law with exponent 3, and as discussed in Boguná et al. (2004b), the structural cutoff and natural cutoff coincide for this model. So, considering either cutoff, for the model considered in this paper, there is an agreement between Equation (46) and our result presented in Equation (47).

\section{Simulation results}

Now we test the theoretical prediction of Equations (25) and (26) through simulations. For all simulations, we use $\alpha=1$, so that at each timestep one new node is added to the network. The first simulation results are presented in Figure 4. In Figure 4(a), the value of $L_{k, \ell}=N_{k \ell}(t)+N_{\ell k}(t)$ is presented, and $p(\ell \mid k)$ is illustrated in Figure 4(b). The initial graph is a 10-regular ring of 50 nodes. It is obtained by situating the nodes on a ring and then connecting each node to its second-, third-, fourth-, and fifth-nearest-neighbor from each side. Then, the growth process with $\beta=2$ begins. In the figure, we show $p(13 \mid 12)$, which provides a representative example. At the outset, the NNDD is zero in this case, because all nodes have the same degree and we have $p(10 \mid 10)=1$ and $p(\ell \mid k)=0$ for any other value of $k, \ell$. As the new nodes are attached to the network, the degrees of the exiting nodes increase and the adjacent nodes of degrees 13 and 12 emerge. The evolution of the system up to $t=140$ is presented. The simulation results are averaged over 2,000 Monte Carlo trials. In Figure 5, the simulation setup is the same, and $k=\ell=10$ is considered. It can be seen that $p(10 \mid 10)$ equals unity at the outset, and it monotonically decreases as new nodes arrive and attach to nodes of degree 10, incrementing their degrees, consequently reducing their population size. The simulation results presented in Figure 6 pertain to the same setup, with $k=10$ and $\ell=2$. It can be seen that at the outset, the value of $p(2 \mid 10)$ is zero, because there is no node with degree 2 , as every node has degree 10. Each new node that arrives, increments the number of degree- 2 nodes by one, and $p(2 \mid 10)$ gradually increases. 


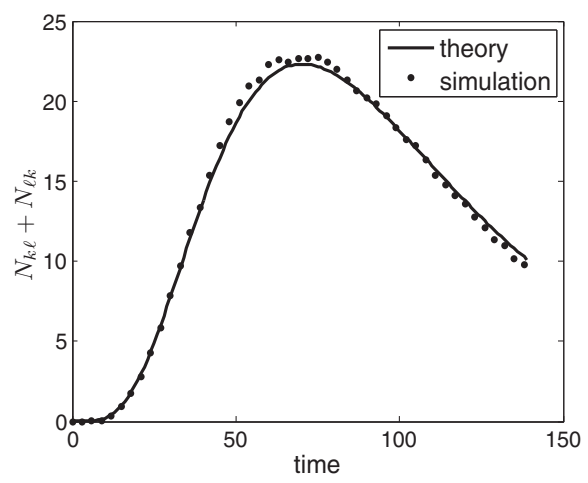

(a) $L_{k \ell}$ as a function of time

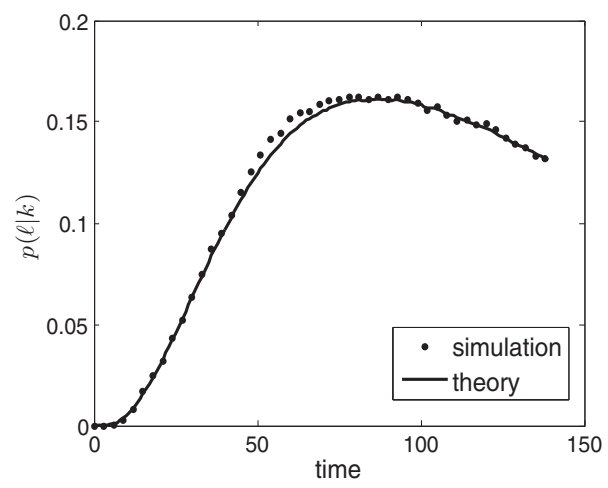

(b) $p(\ell \mid k)$ as a function of time

Fig. 4. Simulation results and theoretical predictions for NNDD and $L_{k, \ell}$. The initial network is a 10-regular ring of 50 nodes. The value of $\beta$ is 2 , also $k=12$ and $\ell=13$. The simulation results are averaged over 2,000 Monte Carlo trials. Theoretical predictions are obtained using (25) and (26). (a) $L_{k \ell}$ as a function of time. (b) $p(\ell \mid k)$ as a function of time.

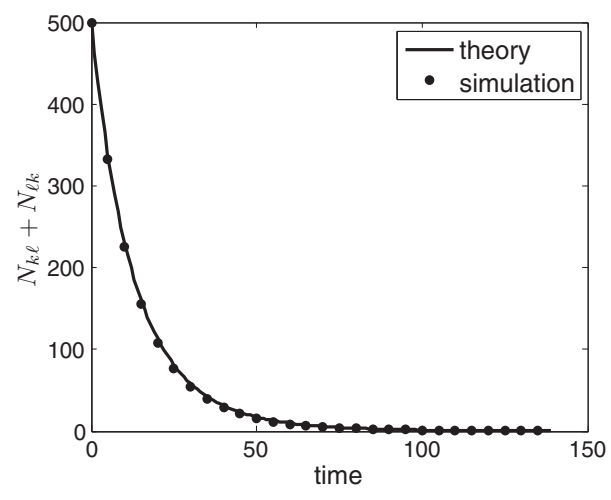

(a) $L_{k \ell}$ as a function of time

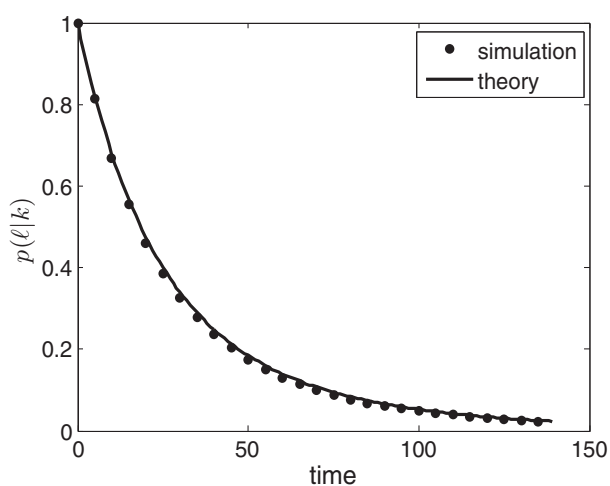

(b) $p(\ell \mid k)$ as a function of time

Fig. 5. Simulation results for the same setup as in Figure 4, except that $k=10$ and $\ell=10$. (a) $L_{k \ell}$ as a function of time. (b) $p(\ell \mid k)$ as a function of time.

In the simulation results presented in Figure 7, the substrate is a complete graph of 20 nodes, and we have focused on the evolution of $p(8 \mid 7)$ as an illustrative example. The value of $\beta$ is 5 . In this case, the value of the NNDD is zero at the inception. The reason is that every node in the initial network has degree 20 at the outset, so none of them can contribute to the constitution of the NNDD. All the contributing nodes are those introduced during the growth process.

In the simulation whose results are depicted in Figure 8 , the initial graph is a 4-regular ring, the value of $\beta$ is $8, k=7$ and $\ell=6$. In this case, every node in the initial substrate has degree 4 , and every subsequent node that joins the network has degree 8 upon birth. So, all the nodes that constitute $p(6 \mid 7)$ are those who were present in the initial substrate, and later gained new links to elevate their degrees from 4 up to 6 and 7. After a while, every node that existed in the initial substrate 


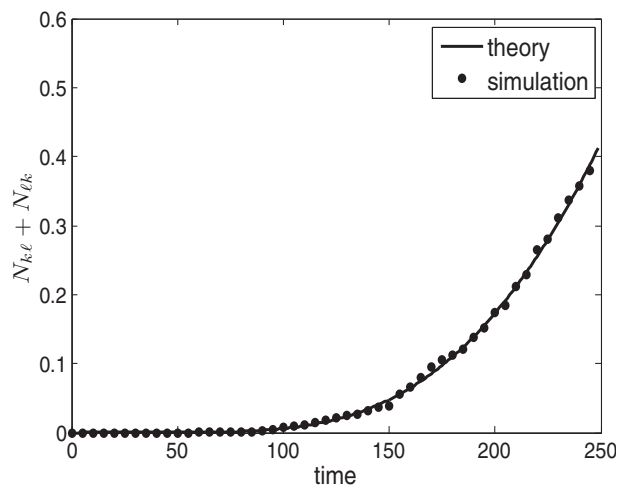

(a) $L_{k \ell}$ as a function of time

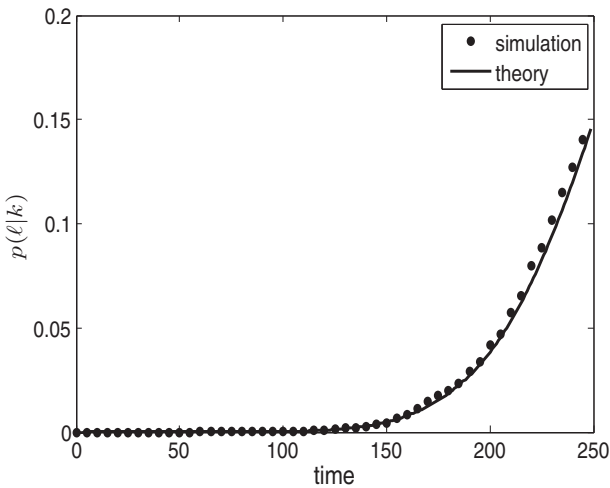

(b) $p(\ell \mid k)$ as a function of time

Fig. 6. Simulation results for the same setup as in Figure 4, except that $k=10$ and $\ell=2$. (a) $L_{k \ell}$ as a function of time. (b) $p(\ell \mid k)$ as a function of time.

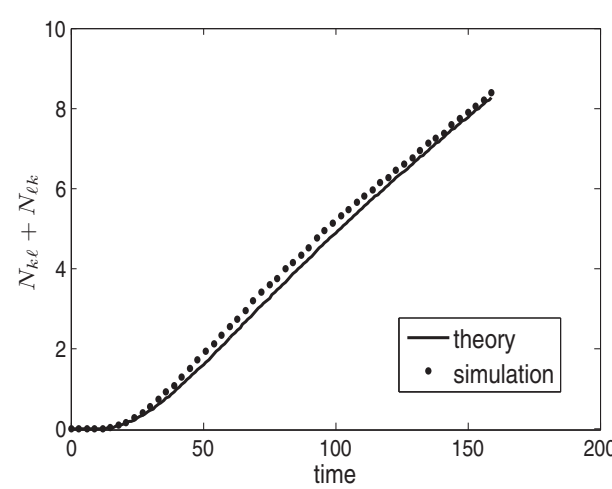

(a) $L_{k \ell}$ as a function of time

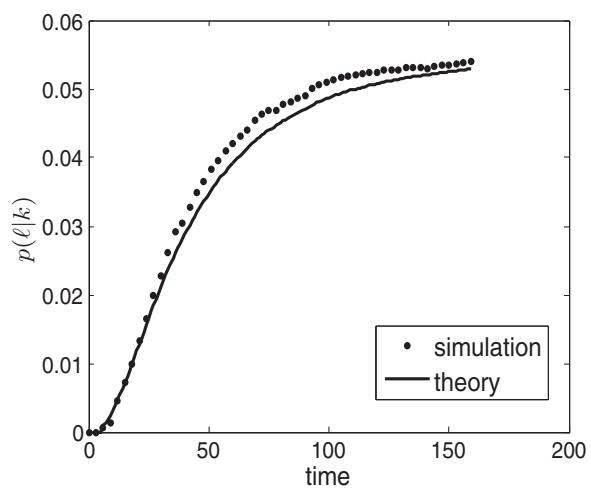

(b) $p(\ell \mid k)$ as a function of time

Fig. 7. Simulation results and theoretical predictions for NNDD and $L_{k, \ell}$. The initial network is a complete graph of 20 nodes. The value of $\beta$ is 5 , also $k=7$ and $\ell=8$. The simulation results are averaged over 2,000 Monte Carlo trials. Theoretical predictions are obtained from (25) and (26). (a) $L_{k \ell}$ as a function of time. (b) $p(\ell \mid k)$ as a function of time.

will receive enough links from the newcomer so that its degree will grow beyond 6 and 7. In fact, after a certain point, the number of nodes with degree 6 and the number of nodes with degree 7 will both monotonically decrease, until they both tend to zero in long times. Consequently, after some point, the value of $L_{7,6}$ will also begin to decrease monotonically and vanish at long times. The simulation results presented in Figure 9 are obtained from the same setup as that of Figure 8. The turning point and the subsequent decrease in Figure 9(a) emerges for similar reasons. However, there is one distinction. In the case considered in Figure 9(a), the decrease in $L_{6,12}$ occurs only due to the fact that the number of nodes of degree 6 is diminishing monotonically. The number of nodes with degree 12 does not decrease monotonically, and does not tend to zero for long times. Figure 10 also pertains to the same setup, with $k=8, \ell=10$. 


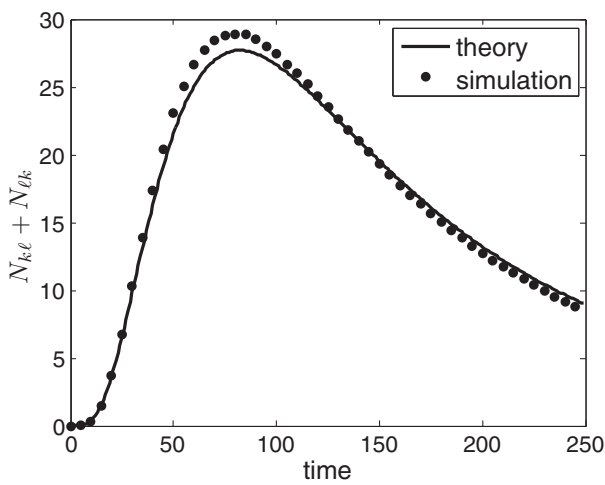

(a) $L_{k \ell}$ as a function of time

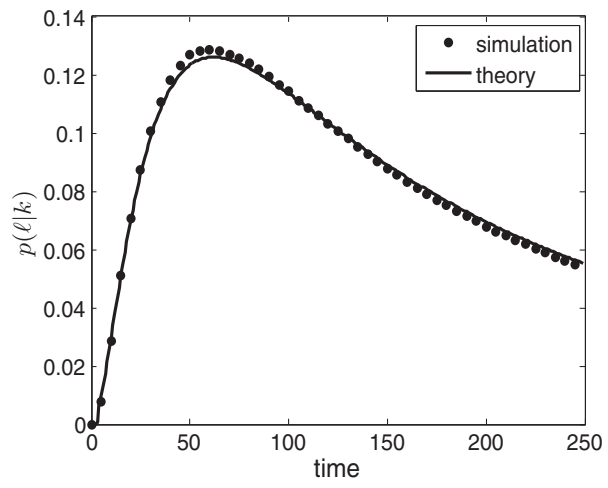

(b) $p(\ell \mid k)$ as a function of time

Fig. 8. Simulation results and theoretical predictions for NNDD and $L_{k, \ell}$. The initial network is a 4-regular ring of 200 nodes. The value of $\beta$ is 8 , also $k=7$ and $\ell=6$. The simulation results are averaged over 2,000 Monte Carlo trials. Theoretical predictions are obtained from (25) and (26). (a) $L_{k \ell}$ as a function of time. (b) $p(\ell \mid k)$ as a function of time.

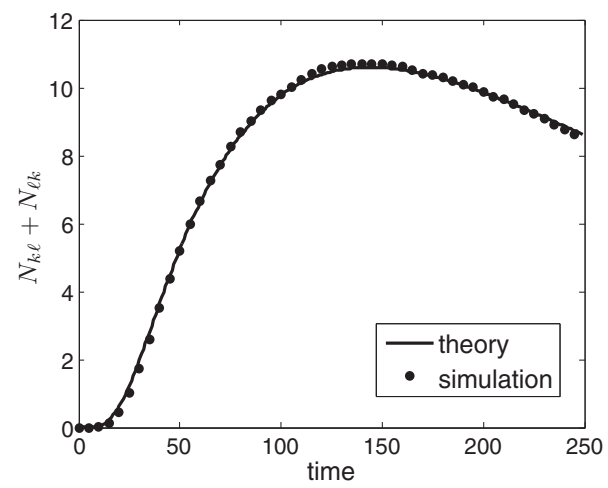

(a) $L_{k \ell}$ as a function of time

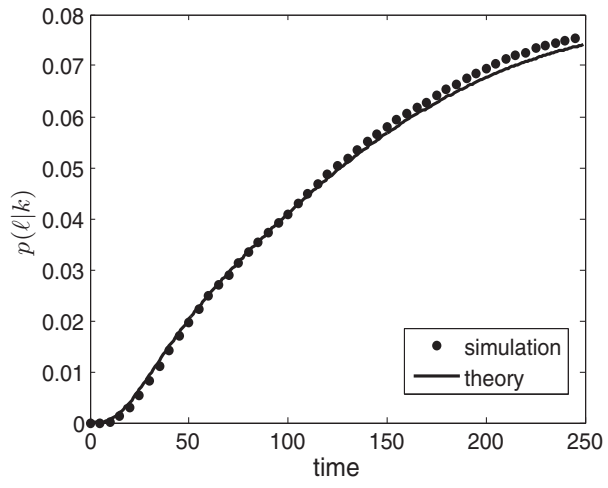

(b) $p(\ell \mid k)$ as a function of time

Fig. 9. Simulation results for the same setup as in Figure 8, except that $k=6$ and $\ell=12$. (a) $L_{k \ell}$ as a function of time. (b) $p(\ell \mid k)$ as a function of time.

To diversify the topologies used for simulations, we present simulation results on two other topologies. The first topology is a small-world. In Figure 11, the initial substrate is constructed by taking a 2-regular ring of 80 nodes and establishing every non-existing link with probability 0.05 . The network begins to grow with $\beta=5$. The results are depicted for $k=8$ and $\ell=6$. The second topology is a complete bipartite graph. It comprises two groups of nodes. There are 50 nodes in the first group and 20 nodes in the other. All possible inter-group links exist, while no intra-group link exists. The values of $k=20$ and $\ell=50$ are used for simulations. The results are depicted in Figure 12.

Let us also verify the steady-state value of the NNDD given in (30) via simulations. The simulation results are presented in Figure 13. The initial network is a 4-regular ring of 20 nodes. The value of $\beta$ is 4 , with $k=6$ and $\ell=8$. We have depicted the evolution of the system up to time 600. It is visible from Figure 13(a) that $N_{k \ell}+N_{\ell k}$ 


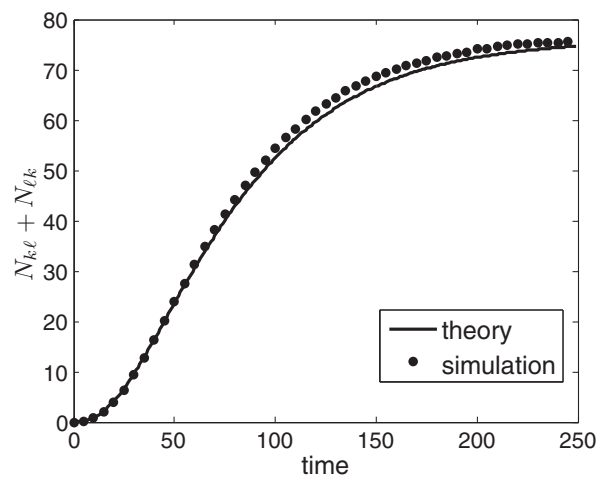

(a) $L_{k \ell}$ as a function of time

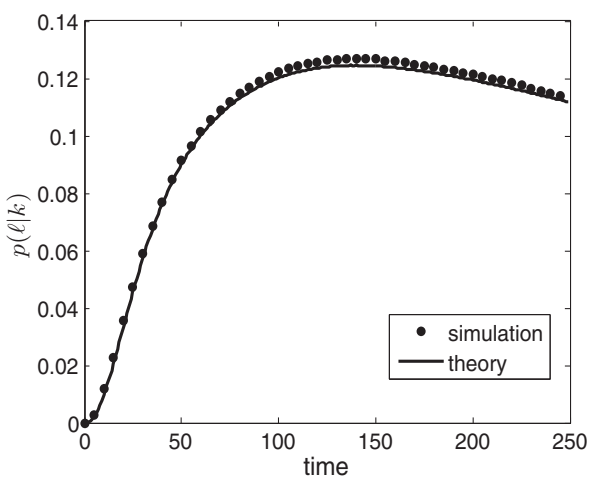

(b) $p(\ell \mid k)$ as a function of time

Fig. 10. Simulation results for the same setup as in Figure 8, except that $k=8$ and $\ell=10$. (a) $L_{k \ell}$ as a function of time. (b) $p(\ell \mid k)$ as a function of time.

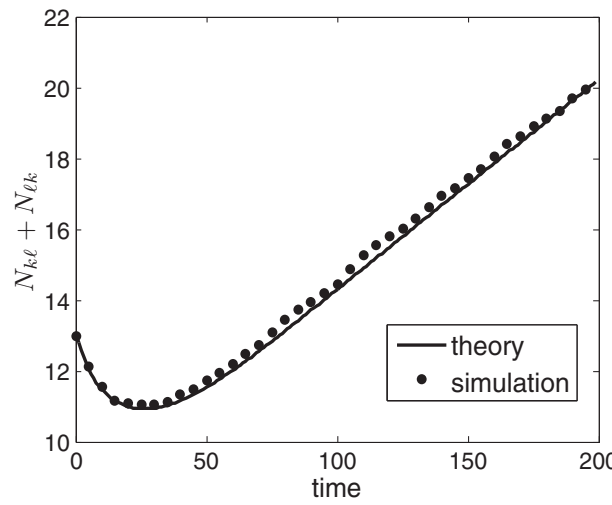

(a) $L_{k \ell}$ as a function of time

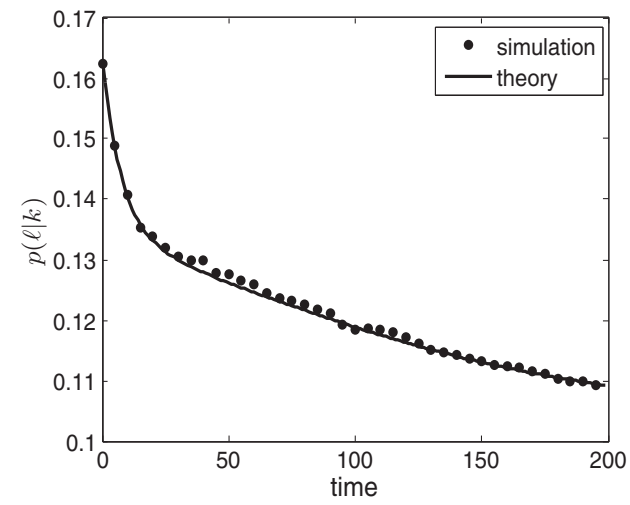

(b) $p(\ell \mid k)$ as a function of time.

Fig. 11. Simulation results for the small-word initial network. The number of nodes in the initial network is 80 . Nodes are situated on a ring, and then each non-existing link is created with probability 0.05 . The value of $\beta$ is 5 , and $k=8$ and $\ell=6$ are used in simulations. Simulation results are averaged over 2,000 Monte Carlo trials. (a) $L_{k \ell}$ as a function of time. (b) $p(\ell \mid k)$ as a function of time.

is linearly growing at long times. This is because, as can be seen from (25), only the first term on the right-hand side survives as $t \rightarrow \infty$, and this term is linear in time.

Finally, Figures 14-17, present the simulation results along with the theoretically predicted mean in a way that the variability of the simulations is also visible. The variance is topology-based and time-dependent.

To further evaluate the quality of our model, we compare its predictions with empirical observations in a citation dataset. We use the network of citations among 34,546 papers posted to the ArXiv high-energy physics phenomenology section between January 1993 and April 2003. ${ }^{4}$ For each paper, we have the date when it was posted as well as the list of out-going edges to other papers cited.

\footnotetext{
4 Available online at http://snap.stanford.edu/data/cit-HepPh.html
} 


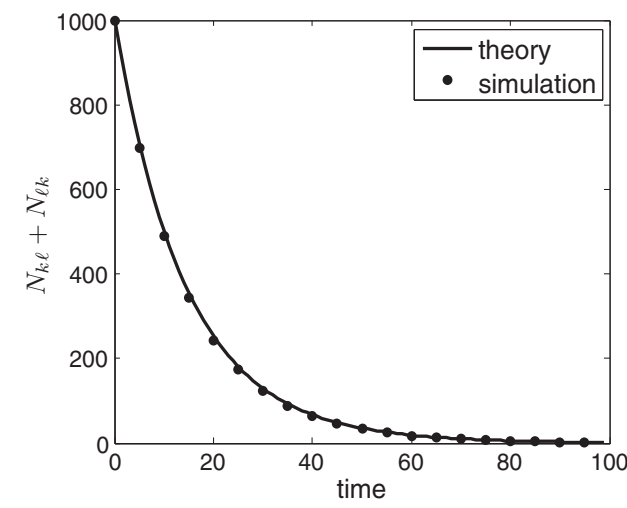

(a) $L_{k \ell}$ as a function of time

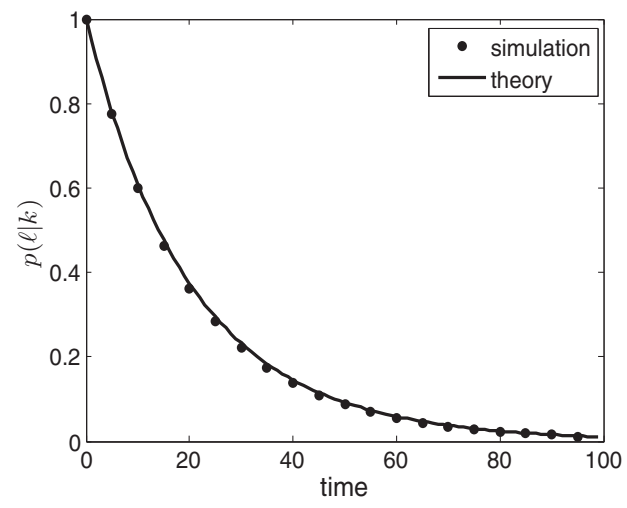

(b) $p(\ell \mid k)$ as a function of time.

Fig. 12. Simulation results for a bipartite graph with 20 nodes in one grouped and 50 nodes on the other. The evolution of the NNDD and $L_{k \ell}$ for the values of $k=20$ and $\ell=50$ is depicted. The value of $\beta$ is 2. Simulation results are averaged over 2,000 Monte Carlo trials. (a) $L_{k \ell}$ as a function of time. (b) $p(\ell \mid k)$ as a function of time.

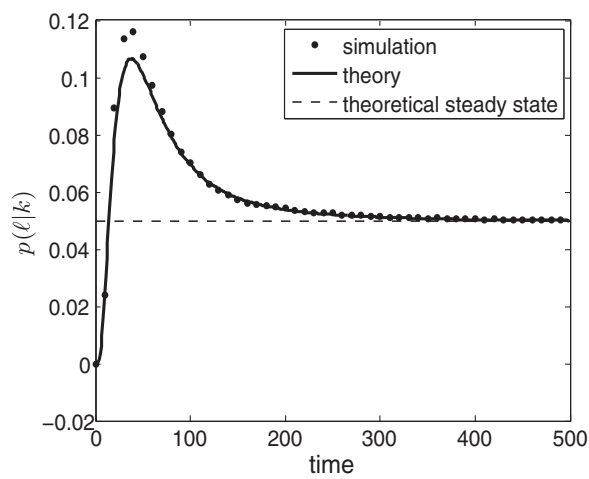

(a) $L_{k \ell}$ as a function of time

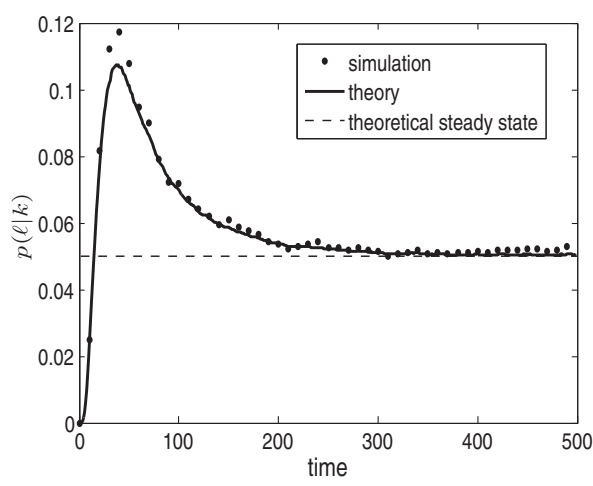

(b) $p(\ell \mid k)$ as a function of time, and the steady state

Fig. 13. Simulation results and theoretical predictions for NNDD and $L_{k, \ell}$. The initial network is a 4-regular ring of 20 nodes. The value of $\beta$ is 4 , also $k=6$ and $\ell=8$. The simulation results are averaged over 2,000 Monte Carlo trials. Theoretical predictions are obtained from (30). The convergence of the NNDD to the theoretical steady-state prediction is visible. Simulation results are averaged over 2,000 Monte Carlo trials. (a) $L_{k \ell}$ as a function of time. (b) $p(\ell \mid k)$ as a function of time, and the steady state.

To evaluate the model proposed in this paper, we perform the following experiment. First, we fix a time $t_{0}$ (e.g., January 1, 1995). The seed graph is formed using all papers before that date, and then we empirically evaluate the NNDD $p(\ell \mid k)$ for the next 1,000 papers posted to the repository. We measure the relative error

$$
\operatorname{RelErr}(\ell \mid k)=\frac{\hat{p}(\ell \mid k)-p(\ell \mid k)}{p(\ell \mid k)}
$$

between the empirical quantity and the estimate $\hat{p}(\ell \mid k)$ from our model with $\alpha=1$ and $\beta$ equal to the mean number of citations considered in the given period. Rather than looking at errors for individual pairs, $(\ell, k)$, we examine the distribution of 


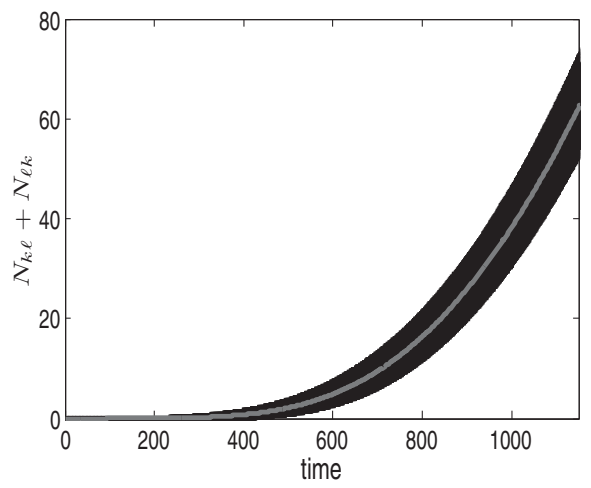

(a) The number of nodes in the initial network is 5000 .

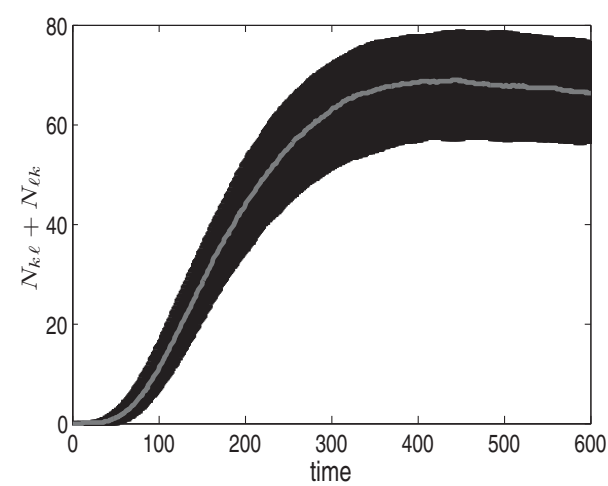

(b) The number of nodes in the initial network is 300

Fig. 14. Theoretical predictions along with the standard deviation of the simulation results. The value of $\beta$ is 4 , with $k=6$ and $\ell=8$. The substrate is a 4-regular ring. Simulation results are averaged over 2,000 Monte Carlo trials. (a) The number of nodes in the initial network is 5,000 . (b) The number of nodes in the initial network is 300 .

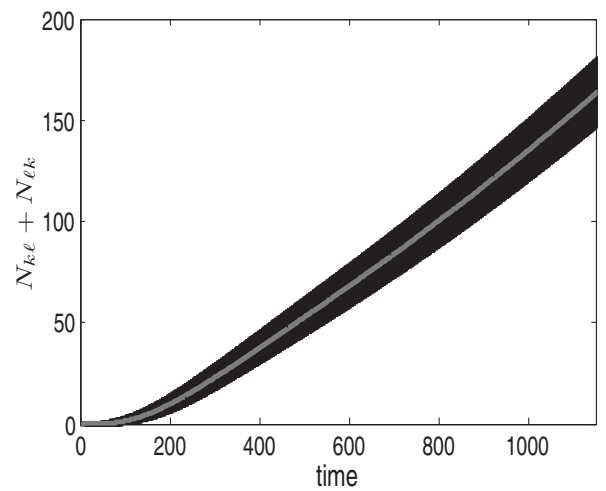

(a) The number of nodes in the initial network is 5000 .

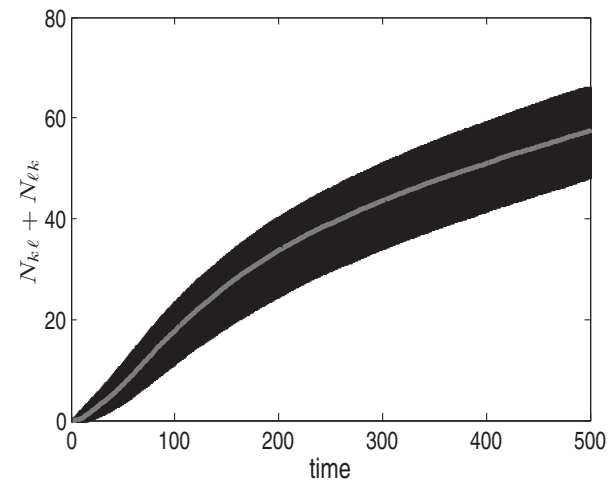

(b) The number of nodes in the initial network is

Fig. 15. Theoretical predictions along with the standard deviation of the simulation results. The value of $\beta$ is 8 , with $k=9$ and $\ell=10$. The substrate is a 2-regular ring. Simulation results are averaged over 2,000 Monte Carlo trials. (a) The number of nodes in the initial network is 5,000. (b) The number of nodes in the initial network is 200 .

$\operatorname{RelErr}(\ell \mid k)$ over the set of pairs for $k$ and $\ell$ ranging from 2 to the 90 th percentile of the degree distribution of the seed graph (which depends on $t_{0}$ ). We then repeat this process for four values of $t_{0}$ (from January 1, 1995, to January 1, 1998) and average the results. The results are shown in Figure 18, where the solid line corresponds to the median relative error, and the shaded region spans from the 25 th to the 75 th percentile. As one might expect, the relative error begins small and progressively grows over time, with the average median error remaining well below $10 \%$ even after 1,000 papers have been added. 


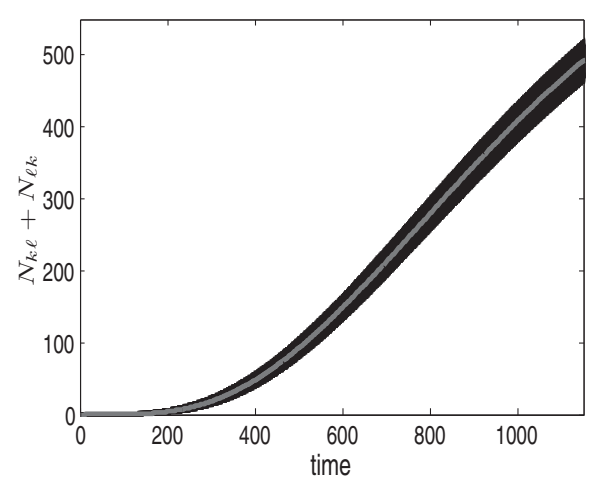

(a) The number of nodes in the initial network is 5000 .

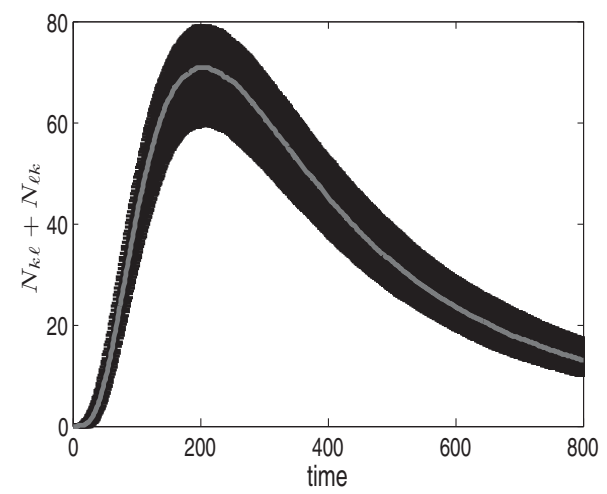

(b) The number of nodes in the initial network is 500 .

Fig. 16. Theoretical predictions along with the standard deviation of the simulation results. The value of $\beta$ is 8 , with $k=7$ and $\ell=6$. The substrate is a 4-regular ring. Simulation results are averaged over 2,000 Monte Carlo trials. (a) The number of nodes in the initial network is 5,000 . (b) The number of nodes in the initial network is 500 .

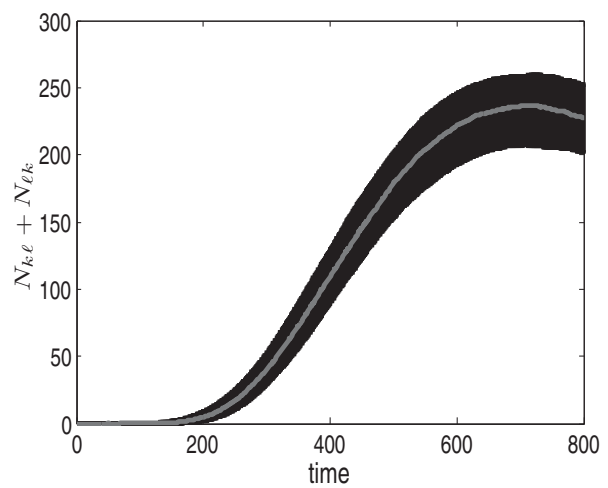

(a) The number of nodes in the initial network is 1000 .

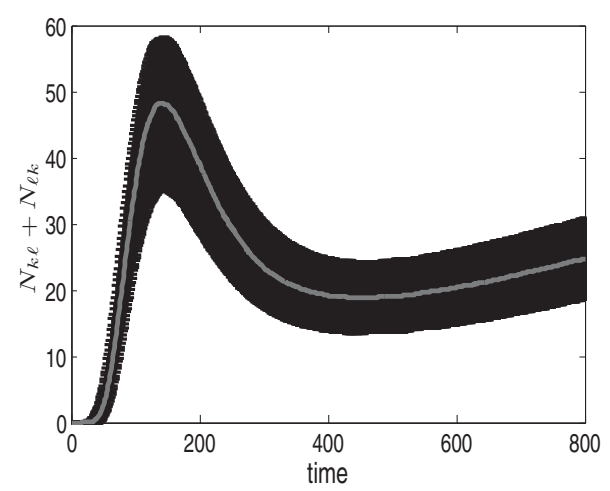

(b) The number of nodes in the initial network is 200.

Fig. 17. Theoretical predictions along with the standard deviation of the simulation results. The value of $\beta$ is 12 , with $k=16$ and $\ell=20$. The substrate is a 12-regular ring. (a) The number of nodes in the initial network is 1,000 . (b) The number of nodes in the initial network is 200 .

\section{Summary and discussion}

This contribution answered the following question: consider a given network with a given degree distribution and a given nearest-neighbors degree distribution. Suppose that the network is subject to growth under the preferential attachment mechanism. How does the nearest-neighbor degree distribution evolve over time?

There is an immense literature on the structural properties of scale-free networks. Most of these results are restricted to the "thermodynamic limit," i.e., when the size of the network tends to infinity. We contended that this unrealistic assumption can render the theory of scale-free networks unfalsifiable, while falsifiability is a necessary property for a scientific model. We argued that the absence of an exact 


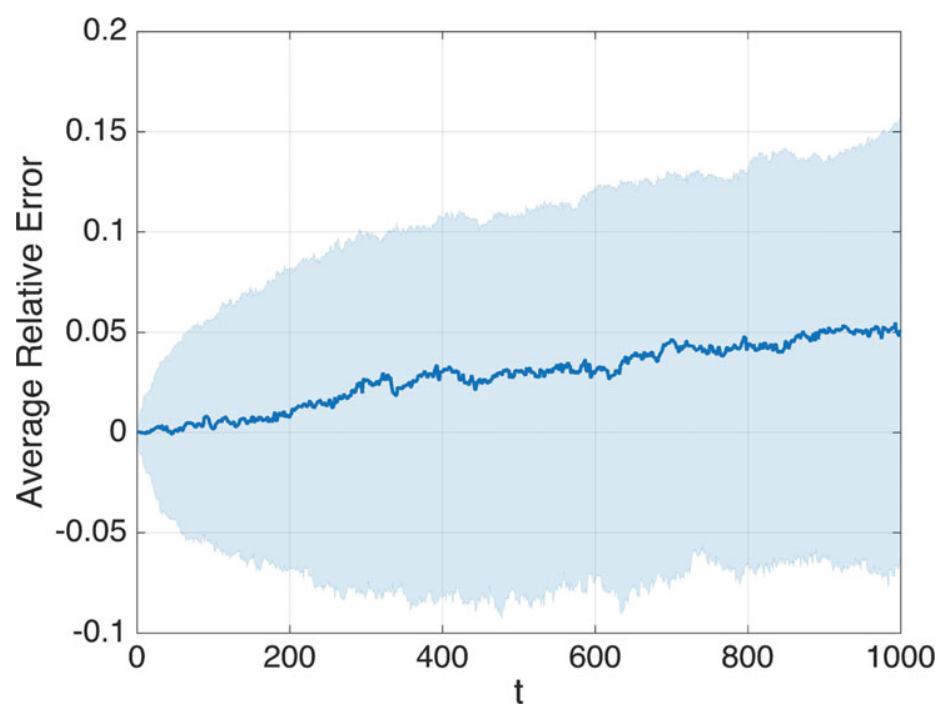

Fig. 18. Distribution of the relative error of the NNDD over a horizon of $T=1,000$ time-steps for different values of $k$ and $\ell$, averaged over eight non-overlapping time windows (roughly corresponding to eight different years). The solid line shows the median relative error, and the shaded region spans from the 25 th to the 75 th percentile. (Color online)

expression for the nearest-neighbor degree distribution, that is invoked frequently in the theoretical analysis of various network phenomena, has confined researches to solutions obtained under uncorrelated approximations, reducing accuracy. Thus, the contribution of this paper can help improve many of the existing results in the literature, and enables us to extend them to intermediary time regimes, beyond the steady-state limit.

There are plausible improvements and extensions to the present work, and to the literature of network growth in general. First, conventional network growth models (such as the one considered in this paper), consider uniform inter-arrival times. One can construct a time-continuous analog of the growth model by modeling node arrival times with a Poisson process.

A second, perhaps methodologically more imperative future work, would be to find the time-evolution of the NNDD for uniformly random network growth, as opposed to the preferential attachment model studied in this paper. When one hypothesizes that there is an underlying mechanism driving the growth of some empirically observable network (example hypotheses include preferential attachment, shiftedlinear and nonlinear generalizations to preferential attachment, fitness-based growth models, copying models, etc.), one requires a null hypothesis. In this context, the null model for network growth would be the absence of any mechanism, which would render the growth purely random. Each new node would attach to the existing nodes selected uniformly at random. The time-evolution of the degree distribution of the purely-random network growth is already obtained in Fotouhi and Rabbat (2013b), but that of the NNDD is still unsolved.

Another plausible extension would be to investigate the time-evolution of the NNDD for shifted-linear preferential attachment, which is a generic framework that encompasses copying and redirection models (see Dorogovtsev et al. (2000), 
Krapivsky and Redner (2001, Sections III-C and III-A3), and Newman (2010, Section 14.5) for examples of models that reduce to shifted-linear scheme). The shifted linear model is more versatile than the one considered in the present paper, since it can engender networks whose asymptotic degree distributions are power laws with exponents arbitrarily greater than 2 .

Note that once the NNDD is known as a function of time, then any quantity that is expressed in terms of the NNDD can be also found as a function of time. For example, in Noh (2008), the number of loops of length $h$ is found as a function of the NNDD. Employing the NNDD obtained in the present paper, we can study the time-dependent loop statistics of the model for a given initial network with given loop statistics. Another example is the threshold value for the transmission rate of pathogen in SIS and SIR models, which are related to the NNDD for large annealed networks, as shown in Barrat et al. (2008) and Boguná et al. (2003), for example. The network of sexual contacts is an example where infection can spread over a growing network. Using the results of this paper, we can obtain sizedependent epidemic thresholds. Another example of an NNDD-dependent quantity is the critical value of the link/node failure, when studying network resilience against random or intentional attacks. As shown in Vázquez and Moreno (2003) and Goltsev et al. (2008), the critical value is related to the largest eigenvalue of a matrix whose elements depend on the NNDD. So, if the network under focus is growing, the time-dependent NNDD is required. Using the NNDD obtained in this paper, one can find the size-dependent percolation threshold to study how network resilience changes over time.

\section{Acknowledgment}

This work was funded in part by the Natural Sciences and Engineering Research Council of Canada, and in part by a grant from the James S. Mcdonnell Foundation. The first author also thanks Naghmeh Momeni for her tremendous help in debugging both the simulation codes and the calculations and also for her persistence in not letting the first author give up.

\section{References}

Albert, R., \& Barabási, A. L. (2000). Topology of evolving networks: Local events and universality. Physical Review Letters, 85(24), 5234.

Apolloni, A., Poletto, C., Ramasco, J. J., Jensen, P., \& Colizza, V. (2014). Metapopulation epidemic models with heterogeneous mixing and travel behaviour. Theoretical Biology and Medical Modelling, 11(1), 3.

Barabási, A. L., \& Albert, R. (1999). Emergence of scaling in random networks. Science, 286(5439), 509-512.

Baronchelli, A., Catanzaro, M., \& Pastor-Satorras, R. (2008). Random walks on complex trees. Physical Review E, 78(1), 011114.

Baronchelli, A., \& Pastor-Satorras, R. (2010). Mean-field diffusive dynamics on weighted networks. Physical Review E, 82(1), 011111.

Barrat, A., Barthelemy, M., \& Vespignani, A. (2008). Dynamical processes on complex networks (p. 193). Cambridge, UK: Cambridge University Press. 
Barrat, A., \& Pastor-Satorras, R. (2005). Rate equation approach for correlations in growing network models. Physical Review E, 71(3), 036127.

Bender, C., \& Orszag, S. (1978). Advanced mathematical methods for scientists and engineers I: Asymptotic methods and perturbation theory. New York: McGraw-Hill.

Bersini, H., Lenaerts, T., \& Santos, F. C. (2006). Growing biological networks: Beyond the gene-duplication model. Journal of Theoretical Biology, 241(3), 488-505.

Bhan, A., Galas, D. J., \& Dewey, T. G. (2002). A duplication growth model of gene expression networks. Bioinformatics, 18(11), 1486-1493.

Boguná, M., Pastor-Satorras, R., \& Vespignani, A. (2003). Epidemic spreading in complex networks with degree correlations. In R. Pastor-Satorras, M. Rubi, \& A. Diaz-Guilera (Eds.), Statistical mechanics of complex networks (p. 127). Berlin, Heidelberg: Springer.

Boguná, M., Pastor-Satorras, R., \& Vespignani, A. (2004a). Cut-offs and finite size effects in scale-free networks. The European Physical Journal B: Condensed Matter and Complex Systems, 38(2), 205-209.

Boguná, M., Pastor-Satorras, R., \& Vespignani, A. (2004b). Cut-offs and finite size effects in scale-free networks. The European Physical Journal B: Condensed Matter and Complex Systems, 38(2), 205-209.

Bollobás, B., et al. (2001). The degree sequence of a scale-free random graph process. Random Structures \& Algorithms, 18(3), 279-290.

Broder, A., Kumar, R., Maghoul, F., Raghavan, P., Rajagopalan, S., Stata, R., ... Wiener, J. (2000). Graph structure in the web. Computer Networks, 33(1), 309-320.

Brown, J. W., Churchill, R. V., \& Lapidus, M. (1996). Complex variables and applications. New York: McGraw-Hill.

Chung, F., Lu, L., Dewey, T. G., \& Galas, D. J. (2003). Duplication models for biological networks. Journal of Computational Biology, 10(5), 677-687.

Colizza, V., \& Vespignani, A. (2008). Epidemic modeling in metapopulation systems with heterogeneous coupling pattern: Theory and simulations. Journal of Theoretical Biology, 251(3), 450-467.

Dorogovtsev, S., Ferreira, A., Goltsev, A., \& Mendes, J. (2010). Zero pearson coefficient for strongly correlated growing trees. Physical Review E, 81(3), 031135.

Dorogovtsev, S. N., \& Mendes, J. F. (2002). Evolution of networks. Advances in Physics, 51(4), 1079-1187.

Dorogovtsev, S. N., Mendes, J. F. F., \& Samukhin, A. N. (2000). Structure of growing networks with preferential linking. Physical Review Letters, 85, 4633.

Faloutsos, M., Faloutsos, P., \& Faloutsos, C. (1999). On power-law relationships of the internet topology. ACM SIGCOMM Computer Communication Review, 29, 251-262.

Ferreira, S. C., Ferreira, R. S., \& Pastor-Satorras, R. (2011). Quasistationary analysis of the contact process on annealed scale-free networks. Physical Review E, 83(6), 066113.

Fortunato, S., Boguñá, M., Flammini, A., \& Menczer, F. (2007). On local estimations of pagerank: A mean field approach. Internet Mathematics, 4(2-3), 245-266.

Fortunato, S., Boguñá, M., Flammini, A., \& Menczer, F. (2008). Approximating pagerank from in-degree. In W. Aiello, A. Broder, J. Janssen, \& E. Milios (Eds.), Proceedings of the 4th international workshop on algorithms and models for the web-graph, WAW'06 (pp. 59-71). Springer.

Fotouhi, B., \& Rabbat, M. (2013a). Degree correlation in scale-free graphs. The European Physical Journal B, 86(12), 1-19.

Fotouhi, B., \& Rabbat, M. (2013b). Network growth with arbitrary initial conditions: Degree dynamics for uniform and preferential attachment. Physical Review E, 88(6), 062801.

Goltsev, A., Dorogovtsev, S., \& Mendes, J. (2008). Percolation on Correlated Networks. Physical Review E, 78, 051105. 
Hasegawa, T., Takaguchi, T., \& Masuda, N. (2013). Observability transitions in correlated networks. Physical Review E, 88(4), 042809.

Holme, P., \& Saramäki, J. (2012). Temporal networks. Physics Reports, 519(3), 97-125.

Jin, E. M., Girvan, M., \& Newman, M. E. (2001). Structure of growing social networks. Physical Review E, 64(4), 046132.

Krapivsky, P., \& Redner, S. (2001). Organization of growing random networks. Physical Review E, 63(6), 066123.

Kumar, R., Novak, J., \& Tomkins, A. (2010). Structure and evolution of online social networks. In P. S. Yu, J. Han, \& C. Faloutsos (Eds.), Link mining: models, algorithms, and applications (pp. 337-357). New York, USA: Springer.

Lee, H., Shim, P.-S., \& Noh, J. (2013). Epidemic threshold of susceptible-infected-susceptible model on complex networks. Physical Review E, 87, 062812.

Marshak, C. Z., Rombach, M. P., Bertozzi, A. L., \& D’Orsogna, M. R. (2016). Growth and containment of a hierarchical criminal network. Physical Review E, 93(2), 022308.

Morse, P. M., \& Feshbach, H. (1953). Methods in theoretical physics, vol. II. New York: McGraw-Hill, (chap. 4).

Newman, M. E. (2002). Assortative mixing in networks. Physical Review Letters, 89(20), 208701.

Newman, M. E. (2004). Coauthorship networks and patterns of scientific collaboration. Proceedings of the National Academy of Sciences of the United States of America, 101(Suppl. 1), 5200-5205.

Newman, M. E. (2010). Networks: An introduction. New York, NY, USA: Oxford University Press.

Noh, J. D. (2008). Loop statistics in complex networks. The European Physical Journal B, 66(2), 251-257.

Olfati-Saber, R., Fax, J. A., \& Murray, R. M. (2007). Consensus and cooperation in networked multi-agent systems. Proceedings of the IEEE, 95(1), 215-233.

Pagani, G. A., \& Aiello, M. (2014). Power grid complex network evolutions for the smart grid. Physica A: Statistical Mechanics and its Applications, 396, 248-266.

Peruani, F. (2009). Advances in the theory of complex networks. In N. Ganguly, A. Deutsch, \& A. Mukherjee (Eds.), Dynamics on and of complex networks (p. 275). Boston, Birkhäuser.

Prakash, B. A., Chakrabarti, D., Faloutsos, M., Valler, N., \& Faloutsos, C. (2010). Got the flu (or mumps)? Check the eigenvalue! arXiv:1004.0060.

Price, D. d. S. (1976). A general theory of bibliometric and other cumulative advantage processes. Journal of the American Society for Information Science, 27(5), 292-306.

Pugliese, E., \& Castellano, C. (2009). Heterogeneous pair approximation for voter models on networks. Europhysics Letters, 88(5), 58004.

Restrepo, J. G., Ott, E., \& Hunt, B. R. (2005). Onset of synchronization in large networks of coupled oscillators. Physical Review E, 71(3), 036151.

Restrepo, J. G., Ott, E., \& Hunt, B. R. (2006). Emergence of coherence in complex networks of heterogeneous dynamical systems. Physical Review Letters, 96(25), 254103.

Restrepo, J. G., Ott, E., \& Hunt, B. R. (2007). Approximating the largest eigenvalue of network adjacency matrices. Physical Review E, 76(5), 056119.

Van Mieghem, P. (2012). Epidemic phase transition of the sis type in networks. Europhysics Letters, 97(4), 48004.

Vázquez, A., \& Moreno, Y. (2003). Resilience to Damage of Graphs with Degree Correlations. Physical Review E, 67(1), 015101.

Vazquez, F., \& Eguíluz, V. M. (2008). Analytical solution of the voter model on uncorrelated networks. New Journal of Physics, 10(6), 063011. 
Zhang, L., Zou, Y., Guan, S., \& Liu, Z. (2015). Analytical description for the critical fixations of evolutionary coordination games on finite complex structured populations. Physical Review E, 91(4), 042807.

Zwillinger, D. (1998). Handbook of differential equations. San Diego, CA: Academic Press.

\section{Appendix A: Finding the explicit form of $K(z, y, t)$}

Let us denote the generating function of the degree distribution of the initial network by $G_{0}(\cdot)$. As shown in Fotouhi and Rabbat (2013b), the time-dependent generating function $G(z, t)$ satisfies

$$
G(z, t)=G_{0}\left(z^{\prime}\right)-\frac{(z-1)^{2}(\lambda+2 \alpha \beta t)}{\beta}\left[F(z)-F\left(z^{\prime}\right)\right]
$$

where $F(z)$ and $z^{\prime}$ are defined as follows $F(z)=\int^{z} \frac{x^{-\beta}}{(x-1)^{3}} d x$, and $z^{\prime}=\frac{z-c}{1-c}$. Note that

$$
\frac{d}{d z} F\left(\frac{z-c}{1-c}\right)=\frac{1}{(1-c)} \frac{\left(\frac{z-c}{1-c}\right)^{-\beta}}{\left(\frac{z-c}{1-c}-1\right)^{3}}=z^{-\beta} \frac{(1-c)^{\beta+2}}{(z-1)^{3}\left(1-\frac{c}{z}\right)^{\beta}}
$$

Also, let us define $z^{\prime} \stackrel{\text { def }}{=} \frac{z-c}{1-c}$. Then, for any function $f\left(z^{\prime}\right)$, we will have $\frac{\partial f\left(z^{\prime}\right)}{\partial z}=$ $\frac{1}{1-c} \frac{\partial f\left(z^{\prime}\right)}{\partial z^{\prime}}$. Taking the derivative of (A1) with respect to $z$, we obtain

$$
\begin{aligned}
\frac{\partial G(z, t)}{\partial z}= & \frac{1}{1-c} \frac{\partial}{\partial z^{\prime}} G_{0}\left(z^{\prime}\right)-\frac{2(z-1)(\lambda+2 \alpha \beta t)}{\beta}\left[F(z)-F\left(z^{\prime}\right)\right] \\
& -\frac{(z-1)^{2}(\lambda+2 \alpha \beta t)}{\beta}\left[\frac{z^{-\beta}}{(z-1)^{3}}-z^{-\beta} \frac{(1-c)^{\beta+2}}{(z-1)^{3}\left(1-\frac{c}{z}\right)^{\beta}}\right]
\end{aligned}
$$

hich can be simplified to

$$
\begin{aligned}
\frac{\partial G(z, t)}{\partial z}= & \frac{1}{1-c} \frac{\partial}{\partial z^{\prime}} G_{0}\left(z^{\prime}\right)-\frac{2(z-1)(\lambda+2 \alpha \beta t)}{\beta}\left[F(z)-F\left(z^{\prime}\right)\right] \\
& -\frac{(\lambda+2 \alpha \beta t)}{\beta(z-1)}\left[z^{-\beta}-z^{-\beta} \frac{(1-c)^{\beta+2}}{\left(1-\frac{c}{z}\right)^{\beta}}\right]
\end{aligned}
$$

Plugging the expression in (A4) into (14), we find

$$
\begin{aligned}
K(z, y, t)= & \frac{-\alpha \beta y^{-\beta}}{(1-c)(\lambda+2 \alpha \beta t)} \frac{\partial}{\partial z^{\prime}} G_{0}\left(z^{\prime}\right)+2 \alpha(z-1) y^{-\beta}\left[F(z)-F\left(z^{\prime}\right)\right] \\
& +\frac{\alpha y^{-\beta}}{(z-1)}\left[z^{-\beta}-z^{-\beta} \frac{(1-c)^{\beta+2}}{\left(1-\frac{c}{z}\right)^{\beta}}\right]
\end{aligned}
$$

\section{Appendix B: Solving the differential equation (16)}

In method of characteristics, the following system of differential equations must be solved:

$$
\frac{d t}{1}=\frac{d z}{-\frac{\alpha \beta(z-1)}{(\lambda+2 \alpha \beta t)}}=\frac{d y}{-\frac{\alpha \beta(y-1)}{(\lambda+2 \alpha \beta t)}}=\frac{d \psi}{K(z, y, t)}
$$


The first equation can be rearranged to give $\frac{\alpha \beta d t}{(\lambda+2 \alpha \beta t)}=\frac{-d z}{z-1}$. Integrating both sides, we obtain

$$
(z-1)^{2}(\lambda+2 \alpha \beta t)=C_{1}
$$

The second equation in (B1) yields $\frac{d z}{z-1}=\frac{d y}{y-1}$. This means that

$$
(z-1)=C_{2}(y-1)
$$

The third equation that we consider from (B1) is $\frac{d z}{-\frac{\alpha \beta(z-1)}{(\lambda+2 \alpha \beta t)}}=\frac{d \psi}{K(z, y, t)}$. This can be expressed equivalently as follows $\frac{-C_{1} d z}{\alpha \beta(z-1)^{3}}=\frac{d \psi}{K(z, y, t)}$. First, we have to express the variables $y, t$ in terms of $z$, to be able to integrate this equation with respect to $z$. From characteristic curves (B2) and (B3), we have

$$
\left\{\begin{array}{l}
y(z)=\frac{z-1}{C_{2}}+1=\frac{z-1+C_{2}}{C_{2}} \\
\lambda+2 \alpha \beta t=\frac{C_{1}}{(z-1)^{2}} \\
t(z)=\frac{C_{1}}{2 \alpha \beta(z-1)^{2}}-\frac{\lambda}{2 \alpha \beta}
\end{array}\right.
$$

Integrating the third equation that involved $K(y, z, t)$ gives

$$
\psi(z, y, t)=\frac{-C_{1}}{\alpha \beta} \int^{z} \frac{K[x, y(x), t(x)]}{(x-1)^{3}} d x+\Phi\left(C_{1}, C_{2}\right)
$$

\section{Appendix C: Finding the inverse transform of (20)}

Now, we can rewrite (20) as

$$
\psi(z, y, t)-\mathscr{2}(z, y, t)=\Phi\left((z-1)^{2}(\lambda+2 \alpha \beta t), \frac{z-1}{y-1}\right)
$$

At $t=0$, the above equation transforms into

$$
\Phi\left(\lambda(z-1)^{2}, \frac{z-1}{y-1}\right)=\psi(z, y, 0)-\mathscr{2}(z, y, 0) .
$$

We are looking for the function $\Phi(\cdot, \cdot)$. If we denote the first and second arguments by $X$ and $Y$, noting that $\lambda$ is a constant, then from $\lambda(z-1)^{2}=X$ we have $z=1+\sqrt{\frac{X}{\lambda}}$, and from $\frac{z-1}{y-1}=Y$ we have $y=1+\frac{1}{Y} \sqrt{\frac{x}{\lambda}}$. To determine the form of the function $\Phi(\cdot, \cdot)$, we rewrite $(\mathrm{C} 2)$ as

$$
\Phi(X, Y)=\psi\left(1+\sqrt{\frac{X}{\lambda}}, 1+\frac{1}{Y} \sqrt{\frac{X}{\lambda}}, 0\right)-\mathscr{Q}\left(1+\sqrt{\frac{X}{\lambda}}, 1+\frac{1}{Y} \sqrt{\frac{X}{\lambda}}, 0\right)
$$

This will yield the last term on the right-hand side of (20). We have

$$
\Phi\left(C_{1}, C_{2}\right)=\psi\left(1+\sqrt{\frac{C_{1}}{\lambda}}, 1+\frac{1}{C_{2}} \sqrt{\frac{C_{1}}{\lambda}}, 0\right)-\mathscr{Q}\left(1+\sqrt{\frac{C_{1}}{\lambda}}, 1+\frac{1}{C_{2}} \sqrt{\frac{C_{1}}{\lambda}}, 0\right)
$$

Now, let us simplify this further. From (6) and (B2), we have $1+\sqrt{\frac{C_{1}}{\lambda}}=1+\frac{z-1}{1-c}=\frac{z-c}{1-c}$. Also, using (B3) we get $1+\frac{1}{C_{2}} \sqrt{\frac{C_{1}}{\lambda}}=1+\left(\frac{y-1}{z-1}\right)\left(\frac{z-1}{1-c}\right)=\frac{y-c}{1-c}$. So, for $\Phi\left(C_{1}, C_{2}\right)$ we arrive at the following form:

$$
\Phi\left(C_{1}, C_{2}\right)=\psi\left(\frac{z-c}{1-c}, \frac{y-c}{1-c}, 0\right)-\mathscr{Q}\left(\frac{z-c}{1-c}, \frac{y-c}{1-c}, 0\right)
$$


If we plug this in (20), we obtain

$$
\psi(z, y, t)=\psi\left(\frac{z-c}{1-c}, \frac{y-c}{1-c}, 0\right)+\left[2(z, y, t)-\mathscr{2}\left(\frac{z-c}{1-c}, \frac{y-c}{1-c}, 0\right)\right]
$$

Now, we focus at finding the inverse Z-transform of each term on the right-hand side of this equation. Consider the first term, which refers to the initial conditions. Denote by $N_{k \ell}(0)$ the number of links in the initial network that connect a child of degree $\ell$ to a parent of degree $k$. Then, by definition, we have $\psi(z, y, 0)=\sum_{r, s} N_{r s}(0) z^{-r} y^{-s}$. The first term on the right-hand side of (C6) will be

$$
\psi\left(\frac{z-c}{1-c}, \frac{y-c}{1-c}, 0\right)=\sum_{r, s} N_{r s}(0)\left(\frac{1-c}{z-c}\right)^{r}\left(\frac{1-c}{y-c}\right)^{s}
$$

The aim is to find the coefficients $b_{k l}$ which satisfy $\psi\left(\frac{z-c}{1-c}, \frac{y-c}{1-c}, 0\right)=\sum_{r, s} b_{r s} z^{-r} y^{-s}$. If we multiply both sides of this equation by $z^{k-1} y^{l-1}$ and integrate over the region of convergence (any closed contour encircling the entire unit disk), we will have

$$
b_{k l}=\left(\frac{1}{2 \pi i}\right)^{2} \oint_{C_{z}} \oint_{C_{y}} \psi\left(\frac{z-c}{1-c}, \frac{y-c}{1-c}, 0\right) z^{k-1} y^{l-1} d z d y
$$

Using (C7), this transforms into

$$
b_{k l}=\left(\frac{1}{2 \pi i}\right)^{2} \sum_{r, s} N_{r s}(0) \oint_{C_{z}}\left(\frac{1-c}{z-c}\right)^{r} z^{k-1} d z \oint_{C_{y}}\left(\frac{1-c}{y-c}\right)^{r} y^{l-1} d y
$$

We define $\sigma_{r k} \stackrel{\text { def }}{=} \frac{1}{2 \pi i} \oint_{C_{z}}\left(\frac{1-c}{z-c}\right)^{r} z^{k-1} d z$. Then, $b_{k l}$ can be expressed in the form

$$
b_{k l}=\sum_{r, s} N_{r s}(0) \sigma_{r k} \sigma_{s l}
$$

We seek to find $\sigma_{r k}$. We must evaluate the following integral $\frac{1}{2 \pi i} \oint_{C_{z}} \frac{z^{k-1}}{(z-c)^{r}} d z$. It equals the residual of the integrand at the pole $z=c$ (Morse and Feshbach, 1953; Brown et al., 1996). When the integrand is of the form $f(z) /(z-p)^{q}$, the residual will be the $(q-1)$-th derivative of the function $f(\cdot)$, evaluated at $z=p$, divided by $(q-1)$ !. So, for the residual for our integrand, which has $f(z)=z^{k-1}$, we obtain

$$
\left.\frac{1}{(r-1) !} \frac{d^{(r-1)}}{d z^{(r-1)}} z^{k-1}\right|_{z=c}= \begin{cases}0 & r>k \\ B_{r-1}^{k-1} c^{k-r} & r \leqslant k\end{cases}
$$

Using this result, we find $\sigma_{r k}$. It equals $\sigma_{r k}=B_{r-1}^{k-1}(1-c)^{r} c^{k-r} u(k-r)$, where $u(x)$ is the Heaviside step function (i.e., $u(x)=0$ for $x<0$, and $u(x)=1$ for $x \geqslant 0$ ). (Note that the binomial coefficient $B_{a}^{b}$ is zero for $b<z$, so it tacitly applies the Heaviside function and the factor $u(b-a)$ is unnecessary.) Employing this expression for $\sigma_{r k}$ in $(\mathrm{C} 10)$, we find

$$
b_{k l}=\sum_{r, s} N_{r s}(0) B_{r-1}^{k-1} B_{s-1}^{\ell-1}\left(\frac{1-c}{c}\right)^{r+s} c^{k+\ell}
$$

This yields the inverse transform of $\psi\left(\frac{z-c}{1-c}, \frac{y-c}{1-c}, 0\right)$, which satisfies (C7). Now, we focus on finding the inverse Z-transform of the second and third terms on the righthand side of (C6). The inverse transform of the term $2\left(\frac{z-c}{1-c}, \frac{y-c}{1-c}, 0\right)$ that appears on the right-hand side of (C6) can be obtained by undertaking identical steps to those 
that led to $(\mathrm{C} 12)$, except that instead of $N_{r s}(0)$ we must use $Q_{r s}(0)$. Plugging these inverse transforms into (C6), we obtain

$$
\begin{aligned}
N_{k \ell}(t)= & Q_{k \ell}(t)+\sum_{r, s} N_{r s}(0) B_{r-1}^{k-1} B_{s-1}^{\ell-1}\left(\frac{1-c}{c}\right)^{r+s} c^{k+\ell} \\
& -\sum_{r, s} Q_{r s}(0) B_{r-1}^{k-1} B_{s-1}^{\ell-1}\left(\frac{1-c}{c}\right)^{r+s} c^{k+\ell}
\end{aligned}
$$

It is easy to verify that at $t=0$, the right-hand side becomes $N_{k \ell}(0)$. Since $c=0$ at this time, all the terms in both sums vanish except for $r=k, s=\ell$. Thus, the first and third terms on the right-hand side of (C13) cancel out, and the second term produces $N_{k \ell}(0)$.

\section{Appendix D: Simplifying $2(z, y, t)$}

Let us repeat the definition of $\mathscr{Z}(z, y, t)$ for easy reference:

$$
\mathscr{2}(z, y, t) \stackrel{\text { def }}{=} \frac{-C_{1}}{\alpha \beta} \int^{z} \frac{K\left[x, \frac{x-1+C_{2}}{C_{2}}, \frac{C_{1}}{2 \alpha \beta(x-1)^{2}}-\frac{\lambda}{2 \alpha \beta}\right]}{(x-1)^{3}} d x
$$

Also, the result of Appendix A, as given in (A5), is the following:

$$
\begin{aligned}
K(z, y, t)= & \frac{-\alpha \beta y^{-\beta}}{(1-c)(\lambda+2 \alpha \beta t)} \frac{\partial}{\partial z^{\prime}} G_{0}\left(z^{\prime}\right)+2 \alpha(z-1) y^{-\beta}\left[F(z)-F\left(z^{\prime}\right)\right] \\
& +\frac{\alpha y^{-\beta}}{(z-1)}\left[z^{-\beta}-z^{-\beta} \frac{(1-c)^{\beta+2}}{\left(1-\frac{c}{z}\right)^{\beta}}\right]
\end{aligned}
$$

There are three terms. Let us simplify each term separately. For easy reference, we define

$$
\begin{aligned}
& K_{1}(z, y, t) \stackrel{\text { def }}{=} \frac{-\alpha \beta y^{-\beta}}{(1-c)(\lambda+2 \alpha \beta t)} \frac{\partial}{\partial z^{\prime}} G_{0}\left(z^{\prime}\right) \\
& K_{2}(z, y, t) \stackrel{\text { def }}{=} 2 \alpha(z-1) y^{-\beta}\left[F(z)-F\left(z^{\prime}\right)\right] \\
& K_{3}(z, y, t) \stackrel{\text { def }}{=} \frac{\alpha y^{-\beta}}{(z-1)}\left[z^{-\beta}-z^{-\beta} \frac{(1-c)^{\beta+2}}{\left(1-\frac{c}{z}\right)^{\beta}}\right]
\end{aligned}
$$

First, let us simplify $K_{1}$. Note that $c$ must be expressed in terms of $z$, since it is a function of time. We have: $c=1-\sqrt{\frac{\lambda}{\lambda+2 \alpha \beta t}}=1-\sqrt{\frac{\lambda}{C_{1}}}(z-1)$. Using this expression, we have

$$
\left\{\begin{array}{l}
z-c=(z-1)\left(1+\sqrt{\frac{\lambda}{C_{1}}}\right) \\
{[-10 p t] 1-c=(z-1) \sqrt{\frac{\lambda}{C_{1}}}} \\
{[-10 p t] \frac{z-c}{1-c}=1+\sqrt{\frac{C_{1}}{\lambda}}}
\end{array}\right.
$$


Plugging these into (D3), we get

$$
K_{1}(z, y, t)=\frac{-\alpha \beta\left[\frac{z-1}{C_{2}}+1\right]^{-\beta}}{(z-1) \sqrt{\frac{\lambda}{C_{1}}}\left(\frac{C_{1}}{(z-1)^{2}}\right)} \frac{\partial}{\partial z^{\prime}} G_{0}\left(z^{\prime}\right)
$$

This can be simplified to give

$$
K_{1}(z, y, t)=\frac{-\alpha \beta C_{2}^{\beta}(z-1)}{\sqrt{\lambda C_{1}}\left(z-1+C_{2}\right)^{\beta}} G_{0}^{\prime}\left(1+\sqrt{\frac{C_{1}}{\lambda}}\right)
$$

Here, the prime on $G_{0}(\cdot)$ denotes the derivative, and is used for brevity. For $K_{2}$, which is given in (D4), expressing $t$ and $y$ in terms of $z$, we get

$$
K_{2}(z, y, t)=2 \alpha(z-1) \frac{C_{2}^{\beta}}{\left(z-1+C_{2}\right)^{\beta}}\left[F(z)-F\left(1+\sqrt{\frac{C_{1}}{\lambda}}\right)\right]
$$

The Taylor expansion of the function $F(\cdot)$ is $F(x)=-\sum_{s=\beta}^{\infty} \frac{B_{2}^{s-\beta}}{s} z^{-s}$. So, we get

$$
K_{2}(z, y, t)=2 \alpha(z-1) \frac{C_{2}^{\beta}}{\left(z-1+C_{2}\right)^{\beta}}\left[-F\left(1+\sqrt{\frac{C_{1}}{\lambda}}\right)-\sum_{s=\beta}^{\infty} \frac{B_{2}^{s-\beta}}{s} z^{-s}\right]
$$

Finally, for $K_{3}$, which is given in (D5), we have

$$
\begin{aligned}
K_{3}(z, y, t)= & \frac{\alpha y^{-\beta}}{(z-1)}\left[z^{-\beta}-z^{-\beta} \frac{(1-c)^{\beta+2}}{\left(1-\frac{c}{z}\right)^{\beta}}\right] \\
= & \frac{\alpha C_{2}^{\beta}}{\left(z-1+C_{2}\right)^{\beta} z^{\beta}(z-1)}-\frac{\alpha C_{2}^{\beta}}{\left(z-1+C_{2}\right)^{\beta}(z-1)}\left[\sqrt{\frac{\lambda}{C_{1}}}\right]^{\beta+2} \\
& \times \frac{(z-1)^{\beta+2}}{(z-1)^{\beta}\left(1+\sqrt{\frac{\lambda}{C_{1}}}\right)^{\beta}} \\
= & \frac{\alpha C_{2}^{\beta}}{\left(z-1+C_{2}\right)^{\beta} z^{\beta}(z-1)}-\frac{\alpha C_{2}^{\beta}}{\left(z-1+C_{2}\right)^{\beta}}\left[\sqrt{\frac{\lambda}{C_{1}}}\right]^{\beta+2} \frac{(z-1)}{\left(1+\sqrt{\frac{\lambda}{C_{1}}}\right)^{\beta}}
\end{aligned}
$$

Now, let us define the following integral: $I_{p q r} \stackrel{\text { def }}{=} \int^{z} \frac{1}{(x-1)^{p}\left(x-1+C_{2}\right)^{q} x^{r}} d x$. Gathering the simplified terms for $K(z)$ that are given in (D8), (D10), and (D11), and plugging them into (19), we get

$$
\begin{aligned}
\mathscr{Q}(z, y, t)= & \frac{2 C_{1} C_{2}^{\beta}}{\beta} \sum_{s=\beta}^{\infty} \frac{B_{2}^{s-\beta}}{s} I_{2 \beta s}+C_{2}^{\beta} \sqrt{\frac{C_{1}}{\lambda}} G_{0}^{\prime}\left(1+\sqrt{\frac{C_{1}}{\lambda}}\right) I_{2 \beta 0} \\
& +\frac{2 C_{1} C_{2}^{\beta}}{\beta} F\left(1+\sqrt{\frac{C_{1}}{\lambda}}\right) I_{2 \beta 0}-\frac{C_{1}}{\beta} C_{2}^{\beta} I_{4 \beta \beta}+\frac{C_{1} C_{2}^{\beta}\left[\sqrt{\frac{\lambda}{C_{1}}}\right]^{\beta+2}}{\beta\left(1+\sqrt{\frac{\lambda}{C_{1}}}\right)^{\beta}} I_{2 \beta 0}
\end{aligned}
$$


To calculate the $I_{p, q, r}$ integrals, let us first expand the following in powers of $x$ before integration: $\frac{1}{(x-1)^{p}\left(x-1+C_{2}\right)^{q} x^{r}}=\sum_{m=0}^{\infty} a_{m}^{p q r} x^{-m}$. The coefficients $a_{k}$ are obtained by multiplying both sides by $x^{k-1}$ and then integrating on a closed path in the complex plane which encircles the origin. So, we have to compute

$$
a_{m}^{p q r}=\frac{1}{2 \pi i} \oint \frac{x^{m-1}}{(x-1)^{p}\left(x-1+C_{2}\right)^{q} x^{r}} d x
$$

To expand the integrand in powers of $z$, we are actually taking the inverse Ztransform. Since multiplication in the $\mathrm{Z}$ domain indicates convolution in the $\mathrm{m}$ domain, we find the inverse transforms separately, then convolve them. We have: $\frac{1}{\left(z-1+C_{2}\right)^{q}} \stackrel{\mathscr{x}^{-1}}{\longrightarrow}\left(1-C_{2}\right)^{m-q} B_{q-1}^{m-1} u(m-q)$, and $\frac{1}{(z-1)^{p} z^{r}} \stackrel{\mathscr{x}^{-1}}{\longrightarrow} B_{p-1}^{m-r-1} u(m-p-r)$. So, we have

$$
a_{m}^{p q r}=\left[B_{p-1}^{m-r-1} *\left(1-C_{2}\right)^{m-q} B_{q-1}^{m-1}\right]=B_{p-1}^{m-r-1} *\left[B_{q-1}^{m-1} \sum_{\mu=0}^{m-q} B_{\mu}^{m-q}(-1)^{\mu} C_{2}^{\mu}\right]
$$

where the asterisks denote convolution. It can be equivalently expressed as follows:

$$
a_{m}^{p q r}=\sum_{\nu=q}^{m-r-1} B_{p-1}^{m-v-r-1}\left[B_{q-1}^{v-1} \sum_{\mu=0}^{v-q} B_{\mu}^{v-q}(-1)^{\mu} C_{2}^{\mu}\right]
$$

Using the expansion, we can now perform the integration and obtain: $I_{p q r}=$ $\sum_{m} \frac{a_{m}^{p q r}}{-m+1} z^{-m+1}$. Since $p+q+r>1$ for all of the terms in (D12), there will be no $\log$ terms as a result of integration. Now, we return to (D12) to substitute for the pertinent integrals. After plugging in the explicit expressions for $C_{1}, C_{2}$, we arrive at

$$
\begin{aligned}
2(z, y, t) & \frac{-2(\lambda+2 \alpha \beta t)}{\beta} \sum_{s=\beta}^{\infty} \frac{B_{2}^{s-\beta}}{s} \sum_{m=\beta+s+2}^{\infty} \frac{1}{(m-1)} \\
& \times \sum_{v=\beta}^{m-s-1} B_{1}^{m-v-s-1} B_{\beta-1}^{v-1} \sum_{\mu=0}^{v-\beta} B_{\mu}^{v-\beta}(-1)^{\mu} \frac{(z-1)^{\mu+\beta+2}}{(y-1)^{\mu+\beta}} z^{-m+1} \\
- & \sqrt{\frac{\lambda+2 \alpha \beta t}{\lambda} G_{0}^{\prime}\left(z^{\prime}\right)} \\
& \times \sum_{m=\beta+2}^{\infty} \frac{1}{(m-1)} \sum_{v=\beta}^{m-1} B_{1}^{m-v-1}\left[B_{\beta-1}^{v-1} \sum_{\mu=0}^{v-\beta} B_{\mu}^{v-\beta}(-1)^{\mu} \frac{(z-1)^{\mu+\beta+1}}{(y-1)^{\mu+\beta}}\right] z^{-m+1} \\
- & \frac{2(\lambda+2 \alpha \beta t)}{\beta} F\left(z^{\prime}\right) \\
& \times \sum_{m=\beta+2}^{\infty} \frac{1}{(m-1)} \sum_{v=\beta}^{m-1} B_{1}^{m-v-1}\left[B_{\beta-1}^{v-1} \sum_{\mu=0}^{v-\beta} B_{\mu}^{v-\beta}(-1)^{\mu} \frac{(z-1)^{\mu+\beta+2}}{(y-1)^{\mu+\beta}}\right] z^{-m+1}
\end{aligned}
$$




$$
\begin{aligned}
+ & \frac{1}{\beta}(\lambda+2 \alpha \beta t) \sum_{m=2 \beta+4}^{\infty} \frac{1}{(m-1)} \\
& \times \sum_{v=\beta}^{m-\beta-1} B_{3}^{m-v-\beta-1}\left[B_{\beta-1}^{v-1} \sum_{\mu=0}^{\nu-\beta} B_{\mu}^{v-\beta}(-1)^{\mu} \frac{(z-1)^{\mu+\beta+2}}{(y-1)^{\mu+\beta}}\right] z^{-m+1} \\
- & \frac{\lambda}{\beta}\left(\frac{\lambda}{\lambda+2 \alpha \beta t}\right)^{\frac{\beta}{2}} \\
& \times \sum_{m=\beta+2}^{\infty} \frac{1}{(m-1)} \sum_{v=\beta}^{m-1} B_{1}^{m-v-1}\left[B_{\beta-1}^{v-1} \sum_{\mu=0}^{v-\beta} B_{\mu}^{v-\beta}(-1)^{\mu} \frac{(z-1)^{\mu+\beta}}{(y-1)^{\mu+\beta}}\right] \frac{z^{-m+1}}{(z-c)^{\beta}}
\end{aligned}
$$

We define $\Gamma_{i \mu}^{m v} \stackrel{\text { def }}{=} B_{i}^{m-v-1} B_{\beta-1}^{v-1} B_{\mu}^{v-\beta}(-1)^{\mu}$ for brevity. Then, (D16) can be written as

$$
\begin{aligned}
\mathscr{2}(z, y, t)= & \frac{-2(\lambda+2 \alpha \beta t)}{\beta} \sum_{s=\beta}^{\infty} \sum_{m=\beta+s+2}^{\infty} \sum_{v=\beta}^{m-s-1} \sum_{\mu=0}^{v-\beta} \frac{B_{2}^{s-\beta}}{s} \frac{\Gamma_{1 \mu}^{(m-s) v}}{(m-1)} \frac{(z-1)^{\mu+\beta+2}}{(y-1)^{\mu+\beta}} z^{-m+1} \\
& -\sqrt{\frac{\lambda+2 \alpha \beta t}{\lambda}} G_{0}^{\prime}\left(z^{\prime}\right) \sum_{m=\beta+2}^{\infty} \sum_{v=\beta}^{m-1} \sum_{\mu=0}^{v-\beta} \frac{\Gamma_{1 \mu}^{m v}}{(m-1)} \frac{(z-1)^{\mu+\beta+1}}{(y-1)^{\mu+\beta}} z^{-m+1} \\
& -\frac{2(\lambda+2 \alpha \beta t)}{\beta} F\left(z^{\prime}\right) \sum_{m=\beta+2}^{\infty} \sum_{v=\beta}^{m-1} \sum_{\mu=0}^{v-\beta} \frac{\Gamma_{1 \mu}^{m v}}{(m-1)} \frac{(z-1)^{\mu+\beta+2}}{(y-1)^{\mu+\beta}} z^{-m+1} \\
& +\frac{1}{\beta}(\lambda+2 \alpha \beta t) \sum_{m=2 \beta+4}^{\infty} \sum_{v=\beta}^{m-\beta-1} \sum_{\mu=0}^{v-\beta} \frac{\Gamma_{3 \mu}^{(m-\beta) v}}{(m-1)} \frac{(z-1)^{\mu+\beta+2}}{(y-1)^{\mu+\beta}} z^{-m+1} \\
& -\frac{\lambda}{\beta}\left(\frac{\lambda}{\lambda+2 \alpha \beta t}\right)^{\frac{\beta}{2}} \sum_{m=\beta+2}^{\infty} \sum_{v=\beta}^{m-1} \sum_{\mu=0}^{v-\beta} \frac{\Gamma_{1 \mu}^{m v}}{(m-1)} \frac{(z-1)^{\mu+\beta}}{(y-1)^{\mu+\beta}} \frac{z^{-m+1}}{(z-c)^{\beta}}
\end{aligned}
$$

\section{Appendix E: Finding the inverse transform of $\mathscr{2}(z, y, t)$}

We now begin taking the inverse transform of $2(z, y, t)$, which is given in (D17), to find $Q_{k}(t)$. To do so, we focus on the individual terms in the right-hand side of (D17) and take their inverse transform one by one. Define

$$
\begin{aligned}
& \mathscr{Q}_{1}(z, y, t) \stackrel{\text { def }}{=} \frac{-2(\lambda+2 \alpha \beta t)}{\beta} \sum_{s=\beta}^{\infty} \sum_{m=\beta+s+2}^{\infty} \sum_{v=\beta}^{m-s-1} \sum_{\mu=0}^{v-\beta} \frac{B_{2}^{s-\beta}}{s} \frac{\Gamma_{1 \mu}^{(m-s) v}}{(m-1)} \frac{(z-1)^{\mu+\beta+2}}{(y-1)^{\mu+\beta}} z^{-m+1} \\
& \mathscr{Q}_{2}(z, y, t) \stackrel{\text { def }}{=}-\sqrt{\frac{\lambda+2 \alpha \beta t}{\lambda}} G_{0}^{\prime}\left(z^{\prime}\right) \sum_{m=\beta+2}^{\infty} \sum_{v=\beta}^{m-1} \sum_{\mu=0}^{v-\beta} \frac{\Gamma_{1 \mu}^{m v}}{(m-1)} \frac{(z-1)^{\mu+\beta+1}}{(y-1)^{\mu+\beta}} z^{-m+1} \\
& \mathscr{Q}_{3}(z, y, t) \stackrel{\text { def }}{=}-\frac{2(\lambda+2 \alpha \beta t)}{\beta} F\left(z^{\prime}\right) \sum_{m=\beta+2}^{\infty} \sum_{v=\beta}^{m-1} \sum_{\mu=0}^{v-\beta} \frac{\Gamma_{1 \mu}^{m v}}{(m-1)} \frac{(z-1)^{\mu+\beta+2}}{(y-1)^{\mu+\beta}} z^{-m+1}
\end{aligned}
$$




$$
\begin{aligned}
& \mathscr{Q}_{4}(z, y, t) \stackrel{\text { def }}{=}+\frac{1}{\beta}(\lambda+2 \alpha \beta t) \sum_{m=2 \beta+4}^{\infty} \sum_{v=\beta}^{m-\beta-1} \sum_{\mu=0}^{v-\beta} \frac{\Gamma_{3 \mu}^{(m-\beta) v}}{(m-1)} \frac{(z-1)^{\mu+\beta+2}}{(y-1)^{\mu+\beta}} z^{-m+1} \\
& \mathscr{Q}_{5}(z, y, t) \stackrel{\text { def }}{=}-\frac{\lambda}{\beta}\left(\frac{\lambda}{\lambda+2 \alpha \beta t}\right)^{\frac{\beta}{2}} \sum_{m=\beta+2}^{\infty} \sum_{v=\beta}^{m-1} \sum_{\mu=0}^{v-\beta} \frac{\Gamma_{1 \mu}^{m v}}{(m-1)} \frac{(z-1)^{\mu+\beta}}{(y-1)^{\mu+\beta}} \frac{z^{-m+1}}{(z-c)^{\beta}}
\end{aligned}
$$

All of these terms involve factors of the form $(z-1)^{a} z^{-b}$, and one can check that in all cases $b>a$, so we have a polynomial of negative powers of $z$, which would be expected, since positive powers would imply negative $k$ and $\ell$. We will use the following general inverse transform, which can be easily verified by expanding the $(z-1)^{a}$ term and then taking the inverse term by term. We have

$$
(z-1)^{a} z^{-b} \stackrel{\mathscr{L}^{-1}}{\longrightarrow} \sum_{\theta=0}^{a} B_{\theta}^{a}(-1)^{\theta-a} \delta[k+\theta-b]
$$

Also, let us temporarily denote the inverse transform of $F\left(z^{\prime}\right)$ and $G^{\prime}\left(z^{\prime}\right)$ by $\hat{f}_{k}$ and $\hat{g}_{k}$, respectively. The explicit expression for these inverse transforms are given in (E63) and (E64). Also, note that the inverse transform of $\frac{1}{(z-c)^{\beta}}$ can be obtained from (7). We arrive at

$$
\begin{aligned}
Q_{k \ell}^{1}(t)= & \frac{-2(\lambda+2 \alpha \beta t)}{\beta} \sum_{s=\beta}^{\infty} \sum_{m=\beta+s+2}^{\infty} \sum_{v=\beta}^{m-s-1} \sum_{\mu=0}^{v-\beta} \sum_{\theta=0}^{\mu+\beta+2} \frac{B_{2}^{s-\beta}}{s} \\
& \times \frac{\Gamma_{1 \mu}^{(m-s) v}}{(m-1)} B_{\mu+\beta-1}^{\ell-1} B_{\theta}^{\mu+\beta+2}(-1)^{\theta+\mu+\beta} \delta[k+\theta-m+1] \\
Q_{k \ell}^{2}(t)= & -\sqrt{\frac{\lambda+2 \alpha \beta t}{\lambda}} \hat{\mathrm{g}}_{k} * \sum_{m=\beta+2}^{\infty} \sum_{v=\beta}^{m-1} \sum_{\mu=0}^{v-\beta} \sum_{\theta=0}^{\mu+\beta+1} \\
& \times \frac{\Gamma_{1 \mu}^{m v}}{(m-1)} B_{\mu+\beta-1}^{\ell-1} B_{\theta}^{\mu+\beta+1}(-1)^{\theta+\mu+\beta+1} \delta[k+\theta-m+1] \\
Q_{k \ell}^{3}(t)= & -\frac{2(\lambda+2 \alpha \beta t)}{\beta} \hat{f}_{k}^{*} \sum_{m=\beta+2}^{\infty} \sum_{v=\beta}^{m-1} \sum_{\mu=0}^{v-\beta} \sum_{\theta=0}^{\mu+\beta+2} \\
& \times \frac{\Gamma_{1 \mu}^{m v}}{(m-1)} B_{\mu+\beta-1}^{\ell-1} B_{\theta}^{\mu+\beta+2}(-1)^{\theta+\mu+\beta} \delta[k+\theta-m+1] \\
Q_{k \ell}^{4}(t)= & +\frac{1}{\beta}(\lambda+2 \alpha \beta t) \sum_{m=2 \beta+4}^{\infty} \sum_{v=\beta}^{m-\beta-1} \sum_{\mu=0}^{v-\beta} \sum_{\theta=0}^{\mu+\beta+2} \\
& \times \frac{\Gamma_{3 \mu}^{(m-\beta) v}}{(m-1)} B_{\mu+\beta-1}^{\ell-1} B_{\theta}^{\mu+\beta+2}(-1)^{\theta+\mu+\beta} \delta[k+\theta-m+1] \\
Q_{k \ell}^{5}(t)= & -\frac{\lambda}{\beta}\left(\frac{\lambda}{\lambda+2 \alpha \beta t}\right)^{\frac{\beta}{2}} \sum_{m=\beta+2}^{\infty} \sum_{v=\beta}^{m-1} \sum_{\mu=0}^{v-\beta} \sum_{\theta=0}^{\mu+\beta} \frac{\Gamma_{1 \mu}^{m v}}{(m-1)} B_{\mu+\beta-1}^{\ell-1} \\
& \times\left[B_{\theta}^{\mu+\beta}(-1)^{\theta+\mu+\beta} \delta[k+\theta-m+1]\right]\left[c^{k-\beta} B_{\beta-1}^{k-1}\right]
\end{aligned}
$$


Changing the index $\mu$ to $\mu+\beta$, and plugging in the explicit expression for $\Gamma$ in every occurrence, this equation transforms into

$$
\begin{aligned}
& Q_{k \ell}^{1}(t)=\frac{-2(\lambda+2 \alpha \beta t)}{\beta} \sum_{s=\beta}^{\infty} \sum_{m=\beta+s+2}^{\infty} \sum_{v=\beta}^{m-s-1} \sum_{\mu=\beta}^{v} \sum_{\theta=0}^{\mu+2} \\
& \times \frac{B_{2}^{s-\beta}}{s} \frac{B_{1}^{m-s-\nu-1} B_{\beta-1}^{v-1} B_{\mu-\beta}^{v-\beta}(-1)^{\theta+\beta}}{(m-1)} B_{\mu-1}^{\ell-1} B_{\theta}^{\mu+2} \delta[k+\theta-m+1] \\
& Q_{k \ell}^{2}(t)=-\sqrt{\frac{\lambda+2 \alpha \beta t}{\lambda}} \hat{\mathrm{g}}_{k} * \sum_{m=\beta+2}^{\infty} \sum_{v=\beta}^{m-1} \sum_{\mu=\beta}^{v} \sum_{\theta=0}^{\mu+1} \\
& \times \frac{B_{1}^{m-\nu-1} B_{\beta-1}^{v-1} B_{\mu-\beta}^{v-\beta}(-1)^{\theta+\beta+1}}{(m-1)} B_{\mu-1}^{\ell-1} B_{\theta}^{\mu+1} \delta[k+\theta-m+1] \\
& Q_{k \ell}^{3}(t)=-\frac{2(\lambda+2 \alpha \beta t)}{\beta} \hat{f}_{k} * \sum_{m=\beta+2}^{\infty} \sum_{v=\beta}^{m-1} \sum_{\mu=\beta}^{v} \sum_{\theta=0}^{\mu+2} \\
& \times \frac{B_{1}^{m-v-1} B_{\beta-1}^{v-1} B_{\mu-\beta}^{v-\beta}(-1)^{\theta+\beta}}{(m-1)} B_{\mu-1}^{\ell-1} B_{\theta}^{\mu+2} \delta[k+\theta-m+1] \\
& Q_{k \ell}^{4}(t)=+\frac{1}{\beta}(\lambda+2 \alpha \beta t) \sum_{m=2 \beta+4}^{\infty} \sum_{\nu=\beta}^{m-\beta-1} \sum_{\mu=\beta}^{\nu} \sum_{\theta=0}^{\mu+2} \\
& \times \frac{B_{3}^{m-\beta-\nu-1} B_{\beta-1}^{v-1} B_{\mu-\beta}^{v-\beta}(-1)^{\theta+\beta}}{(m-1)} B_{\mu-1}^{\ell-1} B_{\theta}^{\mu+2} \delta[k+\theta-m+1] \\
& Q_{k \ell}^{5}(t)=-\frac{\lambda}{\beta}\left(\frac{\lambda}{\lambda+2 \alpha \beta t}\right)^{\frac{\beta}{2}} \sum_{m=\beta+2}^{\infty} \sum_{v=\beta}^{m-1} \sum_{\mu=\beta}^{v} \sum_{\theta=0}^{\mu} \\
& \times \frac{B_{1}^{m-v-1} B_{\beta-1}^{v-1} B_{\mu-\beta}^{v-\beta}(-1)^{\theta+\beta}}{(m-1)} B_{\mu-1}^{\ell-1} B_{\theta}^{\mu} \delta[k+\theta-m+1] *\left(c^{k-\beta} B_{\beta-1}^{k-1}\right)
\end{aligned}
$$

We now simplify these terms separately.

\section{E1 Simplifying $Q_{k \ell}^{1}(t)$}

The first term we consider for simplification is

$$
\begin{aligned}
Q_{k \ell}^{1}(t)= & \frac{-2(\lambda+2 \alpha \beta t)}{\beta} \sum_{s=\beta}^{\infty} \sum_{m=\beta+s+2}^{\infty} \sum_{\nu=\beta}^{m-s-1} \sum_{\mu=\beta}^{v} \sum_{\theta=0}^{\mu+2} \\
& \times \frac{B_{2}^{s-\beta}}{s} \frac{B_{1}^{m-s-\nu-1} B_{\beta-1}^{v-1} B_{\mu-\beta}^{v-\beta}(-1)^{\theta+\beta}}{(m-1)} B_{\mu-1}^{\ell-1} B_{\theta}^{\mu+2} \delta[k+\theta-m+1]
\end{aligned}
$$

Summing over $m$, eliminates this index due to the existence of the delta function. Also, all the summation bounds are automatically imposed by the binomial coefficients, so we can ignore the limits. We have

$$
Q_{k \ell}^{1}(t)=\frac{-2(\lambda+2 \alpha \beta t)}{\beta} \sum_{s=\beta}^{\infty} \sum_{v} \sum_{\mu} \sum_{\theta} \frac{B_{2}^{s-\beta}}{s} \frac{B_{1}^{m-s-v-1} B_{\beta-1}^{\nu-1} B_{\mu-\beta}^{\nu-\beta}(-1)^{\theta+\beta}}{(m-1)} B_{\mu-1}^{\ell-1} B_{\theta}^{\mu+2}
$$


From simple algebraic steps, it follows that

$$
B_{\beta-1}^{v-1} B_{\mu-\beta}^{v-\beta}=\frac{(v-1) !}{(\beta-1) !(v-\beta) !} \frac{(v-\beta) !}{(\mu-\beta) !(v-\mu) !}=\frac{1}{(\beta-1) !} \frac{\mu !}{v} B_{\mu}^{v} \frac{1}{(\mu-\beta) !}=\frac{\beta}{v} B_{\mu}^{v} B_{\beta}^{\mu}
$$

So, (E14) transforms into

$$
Q_{k \ell}^{1}(t)=\frac{-2(\lambda+2 \alpha \beta t)}{\beta} \sum_{s=\beta}^{k+1} \sum_{v=\beta}^{\infty} \sum_{\mu=\beta}^{v} \sum_{\theta=0}^{\mu+2} \frac{B_{2}^{s-\beta}}{s} \frac{(-1)^{\theta+\beta}}{k+\theta} B_{1}^{k+\theta-v-s} \frac{\beta}{v} B_{\mu}^{v} B_{\beta}^{\mu} B_{\mu-1}^{\ell-1} B_{\theta}^{\mu+2}
$$

In Appendix M, we prove that the following holds:

$$
\sum_{v} B_{1}^{k+\theta-s-v} \frac{B_{\mu}^{v}}{v}=\frac{1}{\mu} B_{\mu+1}^{k+\theta-s}
$$

Using this result, summing over the $v$ index in (E16) yields

$$
Q_{k \ell}^{1}(t)=\frac{-2(\lambda+2 \alpha \beta t)}{\beta} \sum_{s=\beta}^{k+1} \sum_{\mu=\beta}^{\infty} \sum_{\theta=0}^{\mu+2} \frac{(-1)^{\theta+\beta}}{k+\theta} B_{\mu+1}^{k+\theta-s} B_{\theta}^{\mu+2} \frac{B_{2}^{s-\beta}}{s} \frac{\beta}{\mu} B_{\beta}^{\mu} B_{\mu-1}^{\ell-1}
$$

In Appendix G, we perform the following summation:

$$
\sum_{\theta} \frac{(-1)^{\theta}}{k+\theta} B_{\mu+1}^{k+\theta-s} B_{\theta}^{\mu+2}=\frac{(-1)^{\mu+1}(\mu+2)}{k(k+1)} \frac{B_{\mu+1}^{\mu+s}}{B_{\mu+1}^{k+\mu+2}}+\frac{(-1)^{\mu}}{k} \delta[s-k-1]
$$

This breaks (E18) into two terms:

$$
\begin{aligned}
Q_{k \ell}^{1}(t)= & -2(\lambda+2 \alpha \beta t)\left[\sum_{s=\beta}^{k+1} \sum_{\mu=\beta}^{\infty} \frac{B_{2}^{s-\beta}}{s} \frac{(-1)^{\mu+\beta+1}}{k(k+1)} \frac{(\mu+2)}{\mu} B_{\mu+1}^{\mu+s} \frac{B_{\beta}^{\mu} B_{\mu-1}^{\ell-1}}{B_{\mu+1}^{k+\mu+2}}\right. \\
& \left.+\sum_{s=\beta}^{k+1} \sum_{\mu=\beta}^{\infty} \frac{B_{2}^{s-\beta}}{s} \frac{(-1)^{\mu+\beta}}{k} \delta[s-k-1] \frac{1}{\mu} B_{\beta}^{\mu} B_{\mu-1}^{\ell-1}\right]
\end{aligned}
$$

We define $\Omega_{\mu} \stackrel{\text { def }}{=} 2 \beta(k+1)+(\beta-k)(\beta+k+1)(\mu+2)+(k-\beta)(k+1-\beta)(\mu+2)^{2}$. In Appendix $\mathrm{H}$, we prove the following identity:

$$
\sum_{s=\beta}^{k+1} \frac{B_{2}^{s-\beta}}{s} B_{\mu+1}^{\mu+s}=\left[\frac{B_{\mu+1}^{\mu+k+2}}{2(\mu+1)(\mu+2)(\mu+3)} \Omega_{\mu}-\frac{B_{\beta-1}^{\mu+\beta+2}}{\mu+1}\right] u(k-\beta-1)
$$

Using this identity in the first term of (E20), and eliminating the delta function in the second term of (E20) by summing over the $s$ index, we find that for $k \leqslant \beta$, the value of $Q_{k \ell}^{1}$ is zero, and for $k \geqslant \beta+1$, it is the following:

$$
\begin{aligned}
Q_{k \ell}^{1}(t)= & -2(\lambda+2 \alpha \beta t)\left\{\sum_{\mu=\beta}^{\ell} \frac{(-1)^{\mu+\beta+1}}{k(k+1)} \frac{(\mu+2)}{\mu(\mu+1)} B_{\beta}^{\mu} B_{\mu-1}^{\ell-1}\right. \\
& \left.\times\left[\frac{\Omega_{\mu}}{2(\mu+2)(\mu+3)}-\frac{B_{\beta-1}^{\mu+\beta+2}}{B_{\mu+1}^{k+\mu+2}}\right]+\sum_{\mu=\beta}^{\infty} B_{2}^{k+1-\beta} \frac{(-1)^{\mu+\beta}}{k(k+1)} \frac{1}{\mu} B_{\beta}^{\mu} B_{\mu-1}^{\ell-1}\right\}
\end{aligned}
$$


Using the definition of $\Omega_{\mu}$, we can use the following decomposition:

$$
\frac{\Omega_{\mu}}{\mu(\mu+1)(\mu+3)}=\frac{2[k(k+1)-3 \beta(k-\beta)]}{3 \mu}-\frac{\beta(\beta+1)}{\mu+1}+\frac{k(k+1)}{3(\mu+3)}
$$

Plugging this into (E20), we arrive at

$$
\begin{aligned}
Q_{k \ell}^{1}(t)= & -(\lambda+2 \alpha \beta t) \sum_{\mu=\beta}^{\ell} \frac{(-1)^{\mu+\beta+1}}{k(k+1)} B_{\beta}^{\mu} B_{\mu-1}^{\ell-1} \\
& \times\left[\frac{2[k(k+1)-3 \beta(k-\beta)]}{3 \mu}-\frac{\beta(\beta+1)}{\mu+1}+\frac{k(k+1)}{3(\mu+3)}\right] \\
& +2(\lambda+2 \alpha \beta t) \sum_{\mu=\beta}^{\ell} \frac{(-1)^{\mu+\beta+1}}{k(k+1)} \frac{(\mu+2)}{\mu(\mu+1)} \frac{B_{\beta}^{\mu} B_{\mu-1}^{\ell-1}}{B_{\mu+1}^{k+\mu+2}} B_{\beta-1}^{\mu+\beta+2} \\
& -2(\lambda+2 \alpha \beta t) \sum_{\mu=\beta}^{\infty} B_{2}^{k+1-\beta} \frac{(-1)^{\mu+\beta}}{k(k+1)} \frac{1}{\mu} B_{\beta}^{\mu} B_{\mu-1}^{\ell-1}
\end{aligned}
$$

In Appendices I-K, the following identities are proven:

$$
\begin{aligned}
& \sum_{\mu} \frac{(-1)^{\mu}}{\mu} B_{\mu-1}^{\ell-1} B_{\beta}^{\mu}=\frac{(-1)^{\ell}}{\ell} \delta[\ell-\beta] \\
& \sum_{\mu} \frac{(-1)^{\mu}}{\mu+1} B_{\mu-1}^{\ell-1} B_{\beta}^{\mu}=\frac{(-1)^{\beta+1}}{\ell(\ell+1)} u(\ell-\beta-1)+\frac{(-1)^{\beta}}{\ell+1} \delta[\ell-\beta] \\
& \sum_{\mu} \frac{(-1)^{\mu}}{\mu+3} B_{\mu-1}^{\ell-1} B_{\beta}^{\mu}=\frac{(-1)^{\beta+1} 3(\beta+1)(\beta+2)}{\ell(\ell+1)(\ell+2)(\ell+3)} u(\ell-\beta-1)+\frac{(-1)^{\beta}}{(\ell+3)} \delta[\ell-\beta]
\end{aligned}
$$

Plugging these results into (E24) leads us to

$$
\begin{aligned}
Q_{k \ell}^{1}(t)= & -(\lambda+2 \alpha \beta t)(\beta+1) \frac{2 k(k+1)+\beta(k-\ell-2)(3+k+\ell)}{k(k+1) \ell(\ell+1)(\ell+2)(\ell+3)} u(\ell-\beta-1) \\
& +(\lambda+2 \alpha \beta t) \frac{2 \beta-(k-\beta)(\beta-2)+(\beta+2)(k-\beta)^{2}}{\beta(\beta+3) k(k+1)} \delta[\ell-\beta] \\
& +2(\lambda+2 \alpha \beta t) \sum_{\mu=\beta}^{\ell} \frac{(-1)^{\mu+\beta+1}}{k(k+1)} \frac{(\mu+2)}{\mu(\mu+1)} \frac{B_{\beta}^{\mu} B_{\mu-1}^{\ell-1}}{B_{\mu+1}^{k+\mu+2}} B_{\beta-1}^{\mu+\beta+2} \\
& -\frac{2(\lambda+2 \alpha \beta t) B_{2}^{k+1-\beta}}{k(k+1)} \frac{(-1)^{\ell+\beta}}{\ell} \delta[\ell-\beta]
\end{aligned}
$$

After some algebraic steps, we obtain the following simplified form:

$$
\begin{aligned}
Q_{k \ell}^{1}(t)= & -(\lambda+2 \alpha \beta t)\left[(\beta+1) \frac{2 k(k+1)+\beta(k-\ell-2)(3+k+\ell)}{k(k+1) \ell(\ell+1)(\ell+2)(\ell+3)} u(\ell-\beta)\right. \\
& \left.-2 \sum_{\mu=\beta}^{\ell} \frac{(-1)^{\mu+\beta+1}}{k(k+1)} \frac{(\mu+2)}{\mu(\mu+1)} \frac{B_{\beta}^{\mu} B_{\mu-1}^{\ell-1}}{B_{\mu+1}^{k+\mu+2}} B_{\beta-1}^{\mu+\beta+2}\right]
\end{aligned}
$$

This must be also multiplied by $u(k-\beta-1)$ because $Q_{k \ell}^{1}$ is zero for $k \leqslant \beta$. 


\section{E2 Simplifying $Q_{k \ell}^{2}(t)$}

Now, we simplify the second term that is given in (E9). We repeat it here for easy reference:

$$
\begin{aligned}
Q_{k \ell}^{2}(t)= & -\sqrt{\frac{\lambda+2 \alpha \beta t}{\lambda}} \hat{\mathrm{g}}_{k} * \sum_{m=\beta+2}^{\infty} \sum_{v=\beta}^{m-1} \sum_{\mu=\beta}^{v} \sum_{\theta=0}^{\mu+1} \\
& \times \frac{B_{1}^{m-v-1} B_{\beta-1}^{v-1} B_{\mu-\beta}^{v-\beta}(-1)^{\theta+\beta+1}}{(m-1)} B_{\mu-1}^{\ell-1} B_{\theta}^{\mu+1} \delta[k+\theta-m+1]
\end{aligned}
$$

First, summing over $m$ and using the properties of the delta function, the index $m$ vanishes and every occurrence of $m$ is replaced by $k+\theta+1$. The result is

$$
Q_{k \ell}^{2}(t)=-\sqrt{\frac{\lambda+2 \alpha \beta t}{\lambda}} \hat{g}_{k} * \sum_{v=\beta}^{\infty} \sum_{\mu=\beta}^{v} \sum_{\theta=0}^{\mu+1} \frac{(-1)^{\theta+\beta+1}}{(k+\theta)} B_{1}^{k+\theta-v} B_{\beta-1}^{v-1} B_{\mu-\beta}^{v-\beta} B_{\mu-1}^{\ell-1} B_{\theta}^{\mu+1}
$$

Using (E15), this can be equivalently expressed as follows:

$$
Q_{k \ell}^{2}(t)=-\sqrt{\frac{\lambda+2 \alpha \beta t}{\lambda}} \hat{g}_{k} * \sum_{v=\beta}^{\infty} \sum_{\mu=\beta}^{v} \sum_{\theta=0}^{\mu+\beta+1} \frac{(-1)^{\theta+\beta+1}}{(k+\theta)} \frac{\beta}{v} B_{1}^{k+\theta-v} B_{\mu}^{v} B_{\beta}^{\mu} B_{\mu-1}^{\ell-1} B_{\theta}^{\mu+1}
$$

Using the identity (E17) for the case of $s=0$, this becomes

$$
Q_{k \ell}^{2}(t)=-\sqrt{\frac{\lambda+2 \alpha \beta t}{\lambda}} \hat{\mathrm{g}}_{k} * \sum_{\mu=\beta}^{\ell} \sum_{\theta=0}^{\mu+\beta+1} \frac{(-1)^{\theta+\beta+1}}{(k+\theta)} \frac{\beta}{\mu} B_{\mu+1}^{k+\theta} B_{\beta}^{\mu} B_{\mu-1}^{\ell-1} B_{\theta}^{\mu+1}
$$

In Appendix L, we prove that

$$
\sum_{\theta} \frac{(-1)^{\theta}}{k+\theta} B_{\mu+1}^{k+\theta} B_{\theta}^{\mu+1}=\frac{(-1)^{\mu+1}}{(\mu+1)} \delta_{k}
$$

thus, $\hat{g}_{k}$ is convolved to a delta function at the origin, which leaves it intact (because $f_{k} * \delta_{k}=f_{k}$, for any discrete function $f(k)$ ). So, (E33) simplifies to

$$
Q_{k \ell}^{2}(t)=-\left(\hat{g}_{k}\right)(-1)^{\beta} \beta\left(\sqrt{\frac{\lambda+2 \alpha \beta t}{\lambda}}\right) \sum_{\mu=\beta}^{\ell} \frac{(-1)^{\mu}}{\mu+1} \frac{1}{\mu} B_{\beta}^{\mu} B_{\mu-1}^{\ell-1}
$$

This can be expanded in the form of partial fractions

$$
\begin{aligned}
Q_{k \ell}^{2}(t)= & -\left(\hat{g}_{k}\right)(-1)^{\beta} \beta\left(\sqrt{\frac{\lambda+2 \alpha \beta t}{\lambda}}\right) \sum_{\mu=\beta}^{\ell} \frac{(-1)^{\mu}}{\mu} B_{\beta}^{\mu} B_{\mu-1}^{\ell-1} \\
& +\left(\hat{g}_{k}\right)(-1)^{\beta} \beta\left(\sqrt{\frac{\lambda+2 \alpha \beta t}{\lambda}}\right) \sum_{\mu=\beta}^{\ell} \frac{(-1)^{\mu}}{\mu+1} B_{\beta}^{\mu} B_{\mu-1}^{\ell-1}
\end{aligned}
$$

Using the identities (E25) and (E26), and following some algebraic steps, this simplifies to

$$
Q_{k \ell}^{2}(t)=-\beta \frac{\hat{g}_{k}}{\ell(\ell+1)}\left(\sqrt{\frac{\lambda+2 \alpha \beta t}{\lambda}}\right) u(\ell-\beta)
$$




\section{E3 Simplifying $Q_{k \ell}^{3}(t)$}

As given in (E10), the third term of $Q_{k \ell}(t)$ is

$$
\begin{aligned}
Q_{k \ell}^{3}(t)= & -\frac{2(\lambda+2 \alpha \beta t)}{\beta} \hat{f}_{k} * \sum_{m=\beta+2}^{\infty} \sum_{v=\beta}^{m-1} \sum_{\mu=\beta}^{v} \sum_{\theta=0}^{\mu+2} \\
& \frac{B_{1}^{m-v-1} B_{\beta-1}^{v-1} B_{\mu-\beta}^{v-\beta}(-1)^{\theta+\beta}}{(m-1)} B_{\mu-1}^{\ell-1} B_{\theta}^{\mu+2} \delta[k+\theta-m+1]
\end{aligned}
$$

Eliminating $m$ by summing over the $\delta$ function, and then using (E15), this can be recast as

$$
Q_{k \ell}^{3}(t)=-\frac{2(\lambda+2 \alpha \beta t)}{\beta} \hat{f}_{k} * \sum_{\nu=\beta}^{\infty} \sum_{\mu=\beta}^{v} \sum_{\theta=0}^{\mu+2} \frac{(-1)^{\theta+\beta}}{(k+\theta)} B_{1}^{k+\theta-v} \frac{\beta}{v} B_{\mu}^{v} B_{\beta}^{\mu} B_{\mu-1}^{\ell-1} B_{\theta}^{\mu+2}
$$

In Appendix M, we show that

$$
\sum_{v} B_{1}^{k+\theta-v} \frac{B_{\mu}^{v}}{v}=\frac{1}{\mu} B_{\mu+1}^{k+\theta}
$$

Using this result, we perform the summation over $v$ in (E39) to obtain

$$
Q_{k \ell}^{3}(t)=-\frac{2(\lambda+2 \alpha \beta t)}{\beta} \hat{f}_{k} * \sum_{\mu=\beta}^{\infty} \sum_{\theta=0}^{\mu+2} \frac{(-1)^{\theta+\beta}}{(k+\theta)} \frac{\beta}{\mu} B_{\mu+1}^{k+\theta} B_{\beta}^{\mu} B_{\mu-1}^{\ell-1} B_{\theta}^{\mu+2}
$$

In Appendix $\mathrm{N}$, we prove that

$$
\sum_{\theta} \frac{(-1)^{\theta}}{(k+\theta)} B_{\mu+1}^{k+\theta} B_{\theta}^{\mu+2}=\frac{(-1)^{\mu+1}}{\mu+1}(\delta[k]-\delta[k+1])
$$

Now, the convolutions of $\hat{f}_{k}$ to the delta functions at the origin and at $k=-1$ give rise to shifts of zero and negative one on the $k$ domain, respectively. From the properties of the delta function it is straightforward to show that for any discrete function $f_{k}$, the convolution $f_{k} * \delta[k+1]$ equals $f_{k+1}$. So, we arrive at

$$
Q_{k \ell}^{3}(t)=\left[\hat{f}_{k}-\hat{f}_{k+1}\right] \frac{2(\lambda+2 \alpha \beta t)}{\beta} \sum_{\mu=\beta}^{\ell} \frac{(-1)^{\mu+\beta}}{(\mu+1)} \frac{\beta}{\mu} B_{\beta}^{\mu} B_{\mu-1}^{\ell-1}
$$

Expanding in the form of partial fractions, this becomes

$$
Q_{k \ell}^{3}(t)=2(\lambda+2 \alpha \beta t)\left[\hat{f}_{k}-\hat{f}_{k+1}\right](-1)^{\beta}\left[\sum_{\mu=\beta}^{\ell} \frac{(-1)^{\mu}}{\mu} B_{\beta}^{\mu} B_{\mu-1}^{\ell-1}-\sum_{\mu=\beta}^{\ell} \frac{(-1)^{\mu}}{\mu+1} B_{\beta}^{\mu} B_{\mu-1}^{\ell-1}\right]
$$

Using identities (E25) and (E26) to evaluate these sums, which are proven in Appendices I and J, respectively, we obtain the following simplified expression:

$$
Q_{k \ell}^{3}(t)=\frac{2(\lambda+2 \alpha \beta t)}{\ell(\ell+1)}\left[\hat{f}_{k}-\hat{f}_{k+1}\right] u(\ell-\beta)
$$




\section{E4 Simplifying $Q_{k \ell}^{4}(t)$}

The fourth term is given in (E11), which is repeated here for easy reference:

$$
\begin{aligned}
Q_{k \ell}^{4}(t)= & +\frac{1}{\beta}(\lambda+2 \alpha \beta t) \sum_{m=2 \beta+4}^{\infty} \sum_{\nu=\beta}^{m-\beta-1} \sum_{\mu=\beta}^{v} \sum_{\theta=0}^{\mu+2} \\
& \times \frac{B_{3}^{m-\beta-v-1} B_{\beta-1}^{v-1} B_{\mu-\beta}^{v-\beta}(-1)^{\theta+\beta}}{(m-1)} B_{\mu-1}^{\ell-1} B_{\theta}^{\mu+2} \delta[k+\theta-m+1]
\end{aligned}
$$

Summing over $m$ to eliminate the delta function and using (E15), this transforms into

$$
Q_{k \ell}^{4}(t)=+\frac{1}{\beta}(\lambda+2 \alpha \beta t) \sum_{v=\beta}^{\infty} \sum_{\mu=\beta}^{v} \sum_{\theta=0}^{\mu+2} \frac{(-1)^{\theta+\beta}}{(k+\theta)} B_{3}^{k+\theta-\beta-v} \frac{\beta}{v} B_{\mu}^{v} B_{\beta}^{\mu} B_{\mu-1}^{\ell-1} B_{\theta}^{\mu+2}
$$

In Appendix $\mathrm{O}$, we show that

$$
\sum_{v} B_{3}^{k+\theta-\beta-v} \frac{B_{\mu}^{v}}{v}=\frac{B_{\mu+3}^{k+\theta-\beta}}{\mu}
$$

This identity helps us sum over $v$ and transform (E47) into

$$
Q_{k \ell}^{4}(t)=+\frac{1}{\beta}(\lambda+2 \alpha \beta t) \sum_{\mu=\beta}^{\infty} \sum_{\theta=0}^{\mu+2} \frac{(-1)^{\theta+\beta}}{(k+\theta)} \frac{\beta}{\mu} B_{\mu+3}^{k+\theta-\beta} B_{\beta}^{\mu} B_{\mu-1}^{\ell-1} B_{\theta}^{\mu+2}
$$

In Appendix $\mathrm{P}$, we prove that

$$
\sum_{\theta} \frac{(-1)^{\theta}}{(k+\theta)} B_{\mu+3}^{k+\theta-\beta} B_{\theta}^{\mu+2}=\frac{(-1)^{\mu}}{\mu+3}\left[1-\frac{B_{\beta-1}^{k-1}}{B_{k-\beta}^{\mu+k+2}}\right] u(k-\beta-1)
$$

Using this identity in (E49) and then taking a partial fraction decomposition, we obtain the following distinct sums:

$$
\begin{aligned}
\left.Q_{k \ell}^{4}(t)\right|_{k \geqslant \beta+1}= & (\lambda+2 \alpha \beta t)\left[\frac{(-1)^{\beta}}{3} \sum_{\mu=\beta}^{\ell} \frac{(-1)^{\mu}}{(\mu)} B_{\beta}^{\mu} B_{\mu-1}^{\ell-1}-\frac{(-1)^{\beta}}{3} \sum_{\mu=\beta}^{\ell} \frac{(-1)^{\mu}}{(\mu+3)} B_{\beta}^{\mu} B_{\mu-1}^{\ell-1}\right. \\
& \left.-\sum_{\mu=\beta}^{\ell} \frac{(-1)^{\beta+\mu}}{(\mu+3)} \frac{1}{\mu} B_{\beta}^{\mu} B_{\mu-1}^{\ell-1} \frac{B_{\beta-1}^{k-1}}{B_{k-\beta}^{\mu+k+2}}\right]
\end{aligned}
$$

Using identities (E25) and (E27), proven in Appendices I and K, respectively, this can be simplified to

$$
\begin{aligned}
Q_{k \ell}^{4}(t)= & (\lambda+2 \alpha \beta t) \frac{(\beta+1)(\beta+2)}{\ell(\ell+1)(\ell+2)(\ell+3)} u(\ell-\beta)-(\lambda+2 \alpha \beta t) \\
& \times \sum_{\mu=\beta}^{\ell} \frac{(-1)^{\beta+\mu}}{(\mu+3)} \frac{1}{\mu} B_{\beta}^{\mu} B_{\mu-1}^{\ell-1} \frac{B_{\beta-1}^{k-1}}{B_{k-\beta}^{\mu+k+2}}
\end{aligned}
$$

This must be also multiplied by the factor $u(k-\beta-1)$, because $Q_{k \ell}^{4}$ is zero for $k \leqslant \beta$. 


\section{E5 Simplifying $Q_{k t}^{5}$}

As given in (E12), the fifth term of $Q_{k \ell}(t)$ is

$$
\begin{aligned}
Q_{k \ell}^{5}(t)= & -\frac{\lambda}{\beta}\left(\frac{\lambda}{\lambda+2 \alpha \beta t}\right)^{\frac{\beta}{2}} \sum_{m=\beta+2}^{\infty} \sum_{v=\beta}^{m-1} \sum_{\mu=\beta}^{v} \sum_{\theta=0}^{\mu} \\
& \times \frac{B_{1}^{m-v-1} B_{\beta-1}^{v-1} B_{\mu-\beta}^{v-\beta}(-1)^{\theta+\beta}}{(m-1)} B_{\mu-1}^{\ell-1} B_{\theta}^{\mu} \delta[k+\theta-m+1] *\left(c^{k-\beta} B_{\beta-1}^{k-1}\right)
\end{aligned}
$$

Similar to the previous terms of $Q_{k}(t)$, we begin by summing over the index $m$ that eliminates the delta function. Then, we use (E15) to obtain

$Q_{k \ell}^{5}(t)=-\frac{\lambda}{\beta}\left(\frac{\lambda}{\lambda+2 \alpha \beta t}\right)^{\frac{\beta}{2}}\left[c^{k-\beta} B_{\beta-1}^{k-1}\right] * \sum_{v=\beta}^{\infty} \sum_{\mu=\beta}^{v} \sum_{\theta=0}^{\mu}\left\{\frac{(-1)^{\theta+\beta}}{(k+\theta)} B_{1}^{k+\theta-v} \frac{\beta}{v} B_{\mu}^{v} B_{\beta}^{\mu} B_{\mu-1}^{\ell-1} B_{\theta}^{\mu}\right.$

Using the identity (E40) and Appendix M, we sum over the index $v$ to obtain

$$
Q_{k \ell}^{5}(t)=-\frac{\lambda}{\beta}\left(\frac{\lambda}{\lambda+2 \alpha \beta t}\right)^{\frac{\beta}{2}}\left[c^{k-\beta} B_{\beta-1}^{k-1}\right] * \sum_{\mu=\beta}^{\ell} \sum_{\theta=0}^{\mu} \frac{(-1)^{\theta+\beta}}{(k+\theta)} \frac{\beta}{\mu} B_{\mu+1}^{k+\theta} B_{\beta}^{\mu} B_{\mu-1}^{\ell-1} B_{\theta}^{\mu}
$$

In Appendix Q, we show that

$$
\sum_{\theta} \frac{(-1)^{\theta}}{(k+\theta)} B_{\mu+1}^{k+\theta} B_{\theta}^{\mu}=\frac{(-1)^{\mu}}{\mu+1} u(k-1)
$$

Note that this sum does not depend on $k$. So, in the $k$ domain, it is a constant. Convolving a discrete function $f_{k}$ by a constant is analogous to discrete integration. For example, convolution of a discrete function $f_{k}$ and unity equals $\sum_{n=0}^{k} f_{n}$. Using this fact, we can rewrite (E55) in the following form:

$$
Q_{k \ell}^{5}(t)=-\lambda(-1)^{\beta}\left(\frac{\lambda}{\lambda+2 \alpha \beta t}\right)^{\frac{\beta}{2}} \sum_{\mu=\beta}^{\ell} \frac{(-1)^{\mu}}{\mu(\mu+1)} B_{\beta}^{\mu} B_{\mu-1}^{\ell-1} \sum_{j=\beta}^{k-1} c^{j-\beta} B_{\beta-1}^{j-1}
$$

After partial fraction decomposition of $\frac{1}{\mu(\mu+1)}$ and using the identities given in (E25) and (E26) that are proven in Appendices I and J, respectively, we obtain

$$
Q_{k \ell}^{5}(t)=-\lambda\left(\frac{\lambda}{\lambda+2 \alpha \beta t}\right)^{\frac{\beta}{2}} \frac{1}{\ell(\ell+1)} u(\ell-\beta) \sum_{j=\beta}^{k-1} c^{j-\beta} B_{\beta-1}^{j-1}
$$

\section{E6 Simplifying $Q_{k \ell}(t)$}

Combining the results obtained in (E29), (E37), (E45), (E52), and (E58) leads us to the full expression for $Q_{k \ell}(t)$. Note that all terms are only nonzero for the range $\ell \geqslant \beta$. For terms with $u(\ell-\beta)$, this follows from the definition of the Heaviside function, and for the terms with sums, since the lower summation bound is $\beta$ and the upper summation index is $\ell$, the sum vanishes if $\ell<\beta$. Hereafter, we will drop the $u(\ell-\beta)$ term for brevity, keeping in mind that $Q_{k \ell}$ is zero for all the values $\ell<\beta$. With this 
consideration, we have

$$
\begin{aligned}
Q_{k \ell}(t)= & -(\lambda+2 \alpha \beta t)\left[(\beta+1) \frac{2 k(k+1)+\beta(k-\ell-2)(3+k+\ell)}{k(k+1) \ell(\ell+1)(\ell+2)(\ell+3)}\right. \\
& \left.-2 \sum_{\mu=\beta}^{\ell} \frac{(-1)^{\mu+\beta+1}}{k(k+1)} \frac{(\mu+2)}{\mu(\mu+1)} \frac{B_{\beta}^{\mu} B_{\mu-1}^{\ell-1}}{B_{\mu+1}^{k+\mu+2}} B_{\beta-1}^{\mu+\beta+2}\right] \\
& -\beta \frac{\hat{g}_{k}}{\ell(\ell+1)}\left(\sqrt{\frac{\lambda+2 \alpha \beta t}{\lambda}}\right)+\frac{2(\lambda+2 \alpha \beta t)}{\ell(\ell+1)}\left[\hat{f}_{k}-\hat{f}_{k+1}\right] \\
& -\lambda\left(\frac{\lambda}{\lambda+2 \alpha \beta t}\right)^{\frac{\beta}{2}} \frac{1}{\ell(\ell+1)} \sum_{j=\beta}^{k-1} c^{j-\beta} B_{\beta-1}^{j-1} \\
& +(\lambda+2 \alpha \beta t) \frac{(\beta+1)(\beta+2)}{\ell(\ell+1)(\ell+2)(\ell+3)} \\
& -(\lambda+2 \alpha \beta t) \sum_{\mu=\beta}^{\ell} \frac{(-1)^{\beta+\mu}}{(\mu+3)} \frac{1}{\mu} B_{\beta}^{\mu} B_{\mu-1}^{\ell-1} \frac{B_{\beta-1}^{k-1}}{B_{k-\beta}^{\mu+k+2}}
\end{aligned}
$$

One can verify the following through straightforward algebraic steps:

$$
\left\{\begin{array}{l}
-(\beta+1) \frac{2 k(k+1)+\beta(k-\ell-2)(3+k+\ell)}{k(k+1) \ell(\ell+1)(\ell+2)(\ell+3)}+\frac{(\beta+1)(\beta+2)}{\ell(\ell+1)(\ell+2)(\ell+3)} \\
=\frac{\beta(\beta+1)}{k(k+1) \ell(\ell+1)} \\
2 \frac{1}{k(k+1)} \frac{(\mu+2)}{\mu(\mu+1)} \frac{B_{\beta}^{\mu} B_{\mu-1}^{\ell-1}}{B_{\mu+1}^{k+\mu+2}} B_{\beta-1}^{\mu+\beta+2}+\frac{1}{(\mu+3)} \frac{1}{\mu} B_{\beta}^{\mu} B_{\mu-1}^{\ell-1} \frac{B_{\beta-1}^{k-1}}{B_{k-\beta}^{\mu+k+2}} \\
=\frac{B_{\beta-1}^{k-1}}{\ell(\ell+1)} \frac{B_{\beta}^{\mu} B_{\mu+1}^{\ell+1}}{B_{k-\beta}^{\mu+k+2}}
\end{array}\right.
$$

This helps us to simplify (E59) and obtain

$$
\begin{aligned}
Q_{k \ell}(t)= & -(\lambda+2 \alpha \beta t)\left[\frac{-\beta(\beta+1)}{k(k+1) \ell(\ell+1)}+\frac{(-1)^{\beta} B_{\beta-1}^{k-1}}{\ell(\ell+1)} \sum_{\mu=\beta}^{\ell}(-1)^{\mu} \frac{B_{\beta}^{\mu} B_{\mu+1}^{\ell+1}}{B_{k-\beta}^{\mu+k+2}}\right] u(k-\beta-1) \\
& -\beta \frac{\hat{g}_{k}}{\ell(\ell+1)}\left(\sqrt{\frac{\lambda+2 \alpha \beta t}{\lambda}}\right)+\frac{2(\lambda+2 \alpha \beta t)}{\ell(\ell+1)}\left[\hat{f}_{k}-\hat{f}_{k+1}\right] \\
& -\lambda\left(\frac{\lambda}{\lambda+2 \alpha \beta t}\right)^{\frac{\beta}{2}} \frac{1}{\ell(\ell+1)} \sum_{j=\beta}^{k-1} c^{j-\beta} B_{\beta-1}^{j-1}
\end{aligned}
$$

The last thing to do is to obtain explicit expressions for $\hat{f}_{k}$ and $\hat{g}_{k}$. Remember that $\hat{f}_{k}$ is the inverse transform of $F\left(z^{\prime}\right)$, where $F(z)$ is the generating function for the function $f_{k}$, defined as $F(z)=\sum_{k} f_{k} z^{-k}$. If we change the argument of $F(\cdot)$ from $z$ to $\frac{z-c}{1-c}$, we have $F\left(\frac{z-c}{1-c}\right)=\sum_{r} f_{r}\left(\frac{1-c}{z-c}\right)^{r}$. The inverse transform is given by

$$
\frac{1}{2 \pi i} \oint \sum_{r} f_{r}\left(\frac{1-c}{z-c}\right)^{r} z^{k-1} d z=\sum_{r} f_{r} \frac{(1-c)^{r}}{2 \pi i} \oint \frac{z^{k-1}}{(z-c)^{r}} d z
$$


Note that the residue of the function $\frac{h(z)}{(z-c)^{r}}$ for a differentiable function $h(\cdot)$ is given by $\frac{h^{(r-1)}}{(r-1) !}$, evaluated at $z=c$. For our problem, $h(z)=z^{k-1}$. So, we have to evaluate the $(r-1)$-th derivative of the function $z^{k-1}$. It is straightforward to see that $\left.\frac{1}{(r-1) !} \frac{d^{(r-1)}}{d z^{(r-1)}} z^{k-1}\right|_{z=c}=c^{k-r} B_{r-1}^{k-1} u(k-r)$. Using this, we evaluate the integrals in (E62) and arrive at

$$
\begin{aligned}
\hat{f}_{k} & =\frac{1}{2 \pi i} \oint \sum_{r} f_{r}\left(\frac{1-c}{z-c}\right)^{r} z^{k-1} d z=\sum_{r=1}^{k} f_{r}(1-c)^{r} c^{k-r} B_{r-1}^{k-1} \\
& =-\sum_{r=1}^{k} \frac{B_{2}^{r-\beta} B_{r-1}^{k-1}}{r}(1-c)^{r} c^{k-r}
\end{aligned}
$$

Since the binomial coefficient is only nonzero for values of $k$ no less than $\beta+2$, we can also change the lower bound of summation from $r=1$ to $r=\beta+2$. We can undertake the steps identical to those above to find the inverse transform of $\hat{g}_{k}$. Note that taking the derivative of a transform of a discrete function $a_{k}$ and then taking the inverse transform is tantamount to taking the transform of the discrete function $-(k-1) a_{k-1}$. Using this fact, we have

$$
\hat{\mathrm{g}}_{k}=-\sum_{r=1}^{k}(r-1) N_{r-1}(0)(1-c)^{r} c^{k-r} B_{r-1}^{k-1}
$$

\section{Appendix F: Obtaining the NNDD from $N_{k \ell}$}

Now, we need to plug $N_{k \ell}(t)$, which is obtained from $(\mathrm{C} 13)$, and $N_{\ell k}(t)$ (which is obtained by exchanging the indices $k$ and $\ell$ ) along with $N_{k}$ (which is given in Equation (5) as a function of time) into (1) to obtain the NNDD that is desired. We have

$$
\begin{aligned}
N_{\ell k}(t)+N_{k \ell}(t)= & Q_{\ell k}(t)+Q_{k \ell}(t)+\sum_{r, s}\left[N_{r s}(0)+N_{s r}(0)\right] B_{r-1}^{k-1} B_{s-1}^{\ell-1}\left(\frac{1-c}{c}\right)^{r+s} c^{k+\ell} \\
& -\sum_{r, s}\left[Q_{r s}(0)+Q_{s r}(0)\right] B_{r-1}^{k-1} B_{s-1}^{\ell-1}\left(\frac{1-c}{c}\right)^{r+s} c^{k+\ell}
\end{aligned}
$$

In the expression for $Q_{\ell k}(t)+Q_{k \ell}(t)$, the left-hand side of the following identity appears:

$$
\begin{gathered}
\frac{B_{\beta-1}^{k-1}}{\ell(\ell+1)} \sum_{\mu=\beta}^{\ell}(-1)^{\mu} \frac{B_{\beta}^{\mu} B_{\mu+1}^{\ell+1}}{B_{k-\beta}^{\mu+k+2}}+\frac{B_{\beta-1}^{\ell-1}}{k(k+1)} \sum_{\mu=\beta}^{k}(-1)^{\mu} \frac{B_{\beta}^{\mu} B_{\mu+1}^{k+1}}{B_{\ell-\beta}^{\mu+\ell+2}} \\
=\beta(\beta+1)(-1)^{\beta} \frac{(k+2) B_{\ell}^{k+\ell+2}+(\ell+1) B_{\beta+1}^{2 \beta+2} B_{k-\beta}^{k+\ell-2 \beta}}{k(k+1)(k+2) \ell(\ell+1) B_{\ell}^{k+\ell+2}}
\end{gathered}
$$

This identity is proven in Appendix R. Using (E61), we find $Q_{k \ell}(0)+Q_{\ell k}(0)$, because it is needed to find $N_{k \ell}(t)+N_{\ell k}(t)$. When $t$ approaches zero, the value of $c$ approaches zero as well. In (E61), the last term has a sum in which different powers of $c$ appear. The same is true in the summations in (E63) and (E64). In all these cases, only one non-zero term exists, in which the power of $c$ is zero, and all terms with positive 
powers of $c$ vanish. After a few simplifications, we arrive at

$$
\begin{aligned}
Q_{k \ell}(0)+Q_{\ell k}(0)= & -\lambda \frac{\beta(\beta+1)}{k(k+1)(k+2) \ell}\left[\frac{k+2}{\ell+1}-\frac{B_{\beta+1}^{2 \beta+2} B_{\ell-\beta}^{k+\ell-2 \beta}}{B_{\ell}^{k+\ell+2}}\right] \\
& -\beta\left[\frac{N_{k}(0)}{\ell(\ell+1)}+\frac{N_{\ell}(0)}{k(k+1)}\right]-\lambda \frac{2 \beta(\beta+1)}{k(k+1) \ell(\ell+1)}
\end{aligned}
$$

Inserting these and (E61) into Equation (F1), and after a few simplifying steps, we arrive at (25). Finally, we must divide this equation by $k N_{k}(t)$ to get $p(\ell \mid k)$. Its value can be obtained by multiplying (5) by $k$.

\section{Appendix G: Proving the identity in Equation (E19)}

We want to show that

$$
\sum_{\theta} \frac{(-1)^{\theta}}{k+\theta} B_{\mu+1}^{k+\theta-s} B_{\theta}^{\mu+2}=\frac{(-1)^{\mu+1}(\mu+2)}{k(k+1)} \frac{B_{\mu+1}^{\mu+s}}{B_{\mu+1}^{k+\mu+2}}+\frac{(-1)^{\mu}}{k} \delta[s-k-1]
$$

Let us define $h_{k} \stackrel{\text { def }}{=} \sum_{\theta} \frac{(-1)^{\theta}}{k+\theta} B_{\mu+1}^{k+\theta-s} B_{\theta}^{\mu+2}$, and $\mathscr{H}(x)=\sum_{k} h_{k} x^{k}$. We have

$$
\begin{aligned}
\mathscr{H}(x) & =\sum_{\theta} x^{-\theta}(-1)^{\theta} B_{\theta}^{\mu+2} \sum_{k} B_{\mu+1}^{k+\theta-s} \frac{x^{k+\theta}}{k+\theta} \\
& =\sum_{\theta} x^{-\theta}(-1)^{\theta} B_{\theta}^{\mu+2} \int^{x} \sum_{k} B_{\mu+1}^{k+\theta-s} x^{k+\theta-1} d x \\
& =\left[\sum_{\theta} x^{-\theta}(-1)^{\theta} B_{\theta}^{\mu+2}\right] \int^{x} x^{s-1} \sum_{k} B_{\mu+1}^{k+\theta-s} x^{k+\theta-s} d x \\
& =\left[\frac{x-1}{x}\right]^{\mu+2} \int^{x} \frac{x^{\mu+s}}{(1-x)^{\mu+2}}
\end{aligned}
$$

Now, let us define $f_{1}(x) \stackrel{\text { def }}{=}\left(\frac{x-1}{x}\right)^{\mu+2}$, and $f_{2}(x) \stackrel{\text { def }}{=} \int \frac{x^{\mu+s}}{(1-x)^{\mu+2}}$. Then, we have $h(x)=$ $f_{1}(x) f_{2}(x)$. Now, let us take the derivative of both sides:

$$
\mathscr{H}^{\prime}(x)=f_{1}^{\prime}(x) f_{2}(x)+f_{1}(x) f_{2}^{\prime}(x)=\frac{f_{1}^{\prime}(x)}{f_{1}(x)} \mathscr{H}(x)+f_{1}(x) f_{2}^{\prime}(x)
$$

It follows from elementary calculus that $f_{1}^{\prime}(x) f_{1}(x)=\frac{-(\mu+2)}{x(1-x)}$, and $f_{1}(x) f_{2}^{\prime}(x)$ $=(-1)^{\mu} x^{s-2}$. So, we arrive at $\mathscr{H}^{\prime}(x)=f_{1}^{\prime}(x) f_{2}(x)+f_{1}(x) f_{2}^{\prime}(x)=\frac{-(\mu+2)}{x(1-x)} \mathscr{H}(x)$ $+(-1)^{\mu} x^{s-2}$. Using the power series representation for $h(x)$ in the form of $\sum_{k} h_{k} x^{k}$ in this equation, we get

$$
(k+1) h_{k+1}=-(\mu+2) u(k+1) * h_{k}+(-1)^{\mu} \delta_{k+2-s}
$$

The convolution with the step function is equivalent to discrete integration, so we get

$$
(k+1) h_{k+1}=-(\mu+2) \sum_{-\infty}^{k+1} h_{k}+(-1)^{\mu} \delta_{k+2-s}
$$


Writing down this equation fork, we get $(k) h_{k}=-(\mu+2) \sum_{-\infty}^{k} h_{k}+(-1)^{\mu} \delta_{k+1-s}$. Subtracting these two equations, we get $(k+1) h_{k+1}-k h_{k}=-(\mu+2) h_{k+1}+(-1)^{\mu}$ $\left[\delta_{k+2-s}-\delta_{k+1-s}\right]$. This can be rearranged and expressed as follows:

$$
h_{k+1}=\frac{k}{\mu+k+3} h_{k}+\frac{(-1)^{\mu}}{\mu+k+3}\left[\delta_{k+2-s}-\delta_{k+1-s}\right]
$$

It is seen from (F4) that for $k=s-2$ the sum is zero. For the next one, we have $h_{s-1}=\frac{(-1)^{\mu}}{\mu+s+1}$. For the next term, we plug $s-1$ for $k$ in (F9) and obtain

$$
\begin{aligned}
h_{s} & =\frac{s-1}{\mu+s+2} \frac{(-1)^{\mu}}{\mu+s+1}-\frac{(-1)^{\mu}}{\mu+s+2}=\frac{(-1)^{\mu}}{\mu+s+2}\left(\frac{s-1}{\mu+s+1}-1\right) \\
& =(-1)^{\mu+1}(\mu+2) \frac{1}{(\mu+s+1)(\mu+s+2)}
\end{aligned}
$$

rom the next term onward, the deltas vanish. We get the recurrence $h_{k+1}=$ $\frac{k}{\mu+k+3} h_{k}, k>s$. So, we get the result through successive multiplications: $h_{k}=$ $h_{s} \prod_{i=s}^{k-1} \frac{i}{\mu+i+3}$. This can be equivalently expressed as follows:

$$
h_{k}=(-1)^{\mu+1} \frac{(\mu+2)}{(\mu+s+1)(\mu+s+2)} \frac{B_{s-1}^{k-1}}{B_{\mu+s+2}^{\mu+k+2}}
$$

By expanding the binomial coefficients, it can be readily verified that

$$
\frac{(\mu+2)}{(\mu+s+1)(\mu+s+2)} \frac{B_{s-1}^{k-1}}{B_{\mu+s+2}^{\mu+k+2}}=\frac{(\mu+2)}{k(k+1)} \frac{B_{\mu+1}^{\mu+s}}{B_{\mu+1}^{k+\mu+2}}
$$

So, we arrive at

$$
h_{k}=\frac{(-1)^{\mu+1}(\mu+2)}{k(k+1)} \frac{B_{\mu+1}^{\mu+s}}{B_{\mu+1}^{k+\mu+2}}
$$

The case of $s=k+1$ is peculiar. In this case, we only have one term, which equals $\frac{(-1)^{\mu}}{\mu+k+2}$, while $(\mathrm{F} 13)$ returns: $\frac{\mu+2}{k} \frac{(-1)^{\mu+1}}{\mu+k+2}$. The difference is $\frac{(-1)^{\mu}}{\mu+k+2}-\frac{\mu+2}{k} \frac{(-1)^{\mu+1}}{\mu+k+2}=$ $\frac{(-1)^{\mu}}{k}$. We add this to compensate for the singular case of $s=k+1$, and obtain

$$
h_{k}=\frac{(-1)^{\mu+1}(\mu+2)}{k(k+1)} \frac{B_{\mu+1}^{\mu+s}}{B_{\mu+1}^{k+\mu+2}}+\frac{(-1)^{\mu}}{k} \delta[s-k-1]
$$

which is the desired result.

\section{Appendix H: Proving the identity in Equation (E21)}

The identity we intend to prove is the following:

$$
\sum_{s=\beta}^{k+1} \frac{B_{2}^{s-\beta}}{s} B_{\mu+1}^{\mu+s}=\left[\frac{B_{\mu+1}^{\mu+k+2}}{2(\mu+1)(\mu+2)(\mu+3)} \Omega_{\mu}-\frac{B_{\beta-1}^{\mu+\beta+2}}{\mu+1}\right] u(k-\beta-1)
$$

with $\Omega_{\mu} \stackrel{\text { def }}{=} 2 \beta(k+1)+(\beta-k)(\beta+k+1)(\mu+2)+(k-\beta)(k+1-\beta)(\mu+2)^{2}$. The step function is justified by noting that in order for the sum to have at least one nonzero term, the binomial coefficient $B_{2}^{s-\beta}$ imposes the condition that $s_{\max } \geqslant \beta+2$. Since $s_{\max }=k+1$, this readily translates into $k \geqslant \beta+1$, hence the step function. 
We use mathematical induction on $k$, for the case of $k=\beta+1$. In this case, only one term in the summation is nonzero, which is $s=\beta+2$, and the left-hand side becomes $\frac{B_{\mu+1}^{\mu+\beta+2}}{\beta+2}$. Also, we have

$$
\Omega_{\mu}=2 \beta(\beta+2)-(2 \beta+2)(\mu+2)+2(\mu+2)^{2}=2[(\mu+1)(\mu+2)-\beta(\mu-\beta)]
$$

Plugging this into the right-hand side of $(\mathrm{H} 1)$ yields

$$
\begin{aligned}
\frac{B_{\mu+1}^{\mu+\beta+3}}{2(\mu+1)(\mu+2)(\mu+3)} 2[(\mu+1)(\mu+2)-\beta(\mu-\beta)]-\frac{B_{\beta-1}^{\mu+\beta+2}}{\mu+1} \\
=\frac{[(\mu+1)(\mu+2)-\beta(\mu-\beta)](\mu+\beta+3) !}{(\mu+1)(\mu+2)(\mu+3)(\mu+1) !(\beta+2) !}-\frac{(\mu+\beta+2) !}{(\mu+1)(\beta-1) !(\mu+3) !} \\
=\frac{(\mu+\beta+2) !}{(\mu+3) !(\mu+1)(\beta+2) !} \\
\quad \times[[(\mu+1)(\mu+2)-\beta(\mu-\beta)](\mu+\beta+3)-\beta(\beta+1)(\beta+2)] \\
=\frac{(\mu+\beta+2) !}{(\mu+3) !(\mu+1)(\beta+2) !}[(\mu+1)(\mu+2)(\mu+3)]=\frac{(\mu+\beta+2) !}{(\mu+1) !(\beta+2) !}=\frac{B_{\mu+1}^{\mu+\beta+2}}{\beta+2}
\end{aligned}
$$

So, the initial step of induction is proven. Now, we focus on the inductive step, namely, showing that if (H1) holds for $k$, then it will also hold for $k+1$. The left-hand side of (H1) for $k+1$ equals

$$
\sum_{s=\beta}^{(k+1)+1} \frac{B_{2}^{s-\beta}}{s} B_{\mu+1}^{\mu+s}=\sum_{s=\beta}^{k+1} \frac{B_{2}^{s-\beta}}{s} B_{\mu+1}^{\mu+s}+\frac{B_{2}^{k+2-\beta}}{k+2} B_{\mu+1}^{\mu+k+2}
$$

The sum can be replaced by the right-hand side of (H1). We get

$$
\sum_{s=\beta}^{(k+1)+1} \frac{B_{2}^{s-\beta}}{s} B_{\mu+1}^{\mu+s}=\left[\frac{B_{\mu+1}^{\mu+k+2}}{2(\mu+1)(\mu+2)(\mu+3)} \Omega_{\mu}-\frac{B_{\beta-1}^{\mu+\beta+2}}{\mu+1}\right]+\frac{B_{2}^{k+2-\beta}}{k+2} B_{\mu+1}^{\mu+k+2}
$$

Using the explicit form of $\Omega_{\mu}$, the induction step reduces to proving the validity of the following:

$$
\begin{aligned}
& \frac{B_{\mu+1}^{\mu+k+2}\left[2 \beta(k+1)+(\beta-k)(\beta+k+1)(\mu+2)+(k-\beta)(k+1-\beta)(\mu+2)^{2}\right]}{2(\mu+1)(\mu+2)(\mu+3)} \\
& -\frac{B_{\beta-1}^{\mu+\beta+2}}{\mu+1}+\frac{B_{2}^{k+2-\beta} B_{\mu+1}^{\mu+k+2}}{k+2} \\
& =\frac{B_{\mu+1}^{\mu+k+3}\left[2 \beta(k+2)+(\beta-k-1)(\beta+k+2)(\mu+2)+(k+1-\beta)(k+2-\beta)(\mu+2)^{2}\right]}{2(\mu+1)(\mu+2)(\mu+3)} \\
& -\frac{B_{\beta-1}^{\mu+\beta+2}}{\mu+1}
\end{aligned}
$$


This can be simplified to

$$
\begin{aligned}
& \frac{B_{\mu+1}^{\mu+k+2}\left[2 \beta(k+1)+(\beta-k)(\beta+k+1)(\mu+2)+(k-\beta)(k+1-\beta)(\mu+2)^{2}\right]}{2(\mu+1)(\mu+2)(\mu+3)} \\
& \quad+\frac{B_{2}^{k+2-\beta} B_{\mu+1}^{\mu+k+2}}{k+2} \\
& =\frac{B_{\mu+1}^{\mu+k+3}\left[2 \beta(k+2)+(\beta-k-1)(\beta+k+2)(\mu+2)+(k+1-\beta)(k+2-\beta)(\mu+2)^{2}\right]}{2(\mu+1)(\mu+2)(\mu+3)}
\end{aligned}
$$

Note that we have $B_{\mu+1}^{\mu+k+3}=\frac{B_{\mu+1}^{\mu+k+2}(\mu+k+3)}{(k+2)}$. Multiplying both sides of (H7) by $2(\mu+1)(\mu+2)(\mu+3)(k+2)$ and dividing both sides by $B_{\mu+1}^{\mu+k+2}$, we arrive at the following, whose validity we must prove in order for the induction step to be concluded:

$$
\begin{aligned}
& {\left[2 \beta(k+1)+(\beta-k)(\beta+k+1)(\mu+2)+(k-\beta)(k+1-\beta)(\mu+2)^{2}\right](k+2)} \\
& \quad+B_{2}^{k+2-\beta} 2(\mu+1)(\mu+2)(\mu+3)-(\mu+k+3)[2 \beta(k+2) \\
& \left.\quad+(\beta-k-1)(\beta+k+2)(\mu+2)+(k+1-\beta)(k+2-\beta)(\mu+2)^{2}\right]=0
\end{aligned}
$$

Let us find the coefficients of different powers of $\mu$. We will show that all these coefficients are zero. Starting with $\mu^{3}$, the coefficient is $2 B_{2}^{k+2-\beta}-(k+1-\beta)(k+2-\beta)$, which equals zero. The coefficient of $\mu^{2}$ is

$$
\begin{aligned}
& {[(k-\beta)(k+1-\beta)(k+2)]+\left[12 B_{2}^{k+2-\beta}\right]} \\
& \quad-[(\beta-k-1)(\beta+k+2)-(k+3)(k+1-\beta)(k+2-\beta)] \\
& \quad=(k+1-\beta)[(k-\beta)(k+2)+6(k+2-\beta)-(k+2+\beta)-(k+3)(k+2-\beta)] \\
& \quad=(k+1-\beta)\left[k^{2}+2 k-\beta k-2 \beta+12-6 \beta+6 k-12+8 \beta-8 k+\beta k-k^{2}\right]=0
\end{aligned}
$$

The coefficient of $\mu$ is

$$
\begin{aligned}
& \left(-6 \beta+10 \beta^{2}+6 k-19 \beta k+5 \beta^{2} k+9 k^{2}-8 \beta k^{2}+3 k^{3}\right) \\
& \quad+\left(22-33 \beta+11 \beta^{2}+33 k-22 \beta k+11 k^{2}\right) \\
& \quad+\left(-22+39 \beta-21 \beta^{2}-39 k+41 \beta k-5 \beta^{2} k-20 k^{2}+8 \beta k^{2}-3 k^{3}\right)=0
\end{aligned}
$$

Finally, the coefficient of $\mu^{0}$ equals

$$
\begin{aligned}
& \left(12 \beta^{2}+4 k-12 \beta k+6 \beta^{2} k+6 k^{2}-6 \beta k^{2}+2 k^{3}\right) \\
& \quad+\left(12-18 \beta+6 \beta^{2}+18 k-12 \beta k+6 k^{2}\right) \\
& \quad+\left(-12+18 \beta-18 \beta^{2}-22 k+24 \beta k-6 \beta^{2} k-12 k^{2}+6 \beta k^{2}-2 k^{3}\right)=0
\end{aligned}
$$

So, the coefficients of every power of $\mu$ is zero. This means that (H8) holds. Hence, the induction step is concluded, and the proof is complete. 


\section{Appendix I: Proving the identity in Equation (E25)}

We want to show that

$$
\sum_{\mu} \frac{(-1)^{\mu}}{\mu} B_{\mu-1}^{\ell-1} B_{\beta}^{\mu}=\frac{(-1)^{\ell}}{\ell} \delta[\ell-\beta]
$$

The left-hand side is a special case of the following sum: $\sum_{\mu} \frac{x^{\mu}}{\mu} B_{\mu-1}^{\ell-1} B_{\beta}^{\mu}$, evaluated at $x=-1$. Note that this sum is equivalent to $\int^{x} \sum_{\mu} x^{\mu-1} B_{\mu-1}^{\ell-1} B_{\beta}^{\mu} d x$.

Let us define $f_{\beta} \stackrel{\text { def }}{=} \int^{x} \sum_{\mu} x^{\mu-1} B_{\mu-1}^{\ell-1} B_{\beta}^{\mu} d x$, and $\mathscr{F}(w) \stackrel{\text { def }}{=} \sum_{\beta} f_{\beta} w^{\beta}$. We can find $f(w)$ by interchanging the order of summation as follows:

$$
\begin{aligned}
\mathscr{F}(w) & =\int^{x} \sum_{\mu} x^{\mu-1} B_{\mu-1}^{\ell-1} \sum_{\beta} B_{\beta}^{\mu} w^{\beta} d x=\int^{x} \sum_{\mu} x^{\mu-1} B_{\mu-1}^{\ell-1}(1+w)^{\mu} d x \\
& =(1+w) \int^{x} \sum_{\mu} B_{\mu-1}^{\ell-1}[x(1+w)]^{\mu-1} d x=(1+w) \int^{x}[1+x(1+w)]^{\ell-1} d x \\
& =\frac{[1+x(1+w)]^{\ell}}{\ell}
\end{aligned}
$$

Evaluating this at $x=-1$ to get the original sum, we get $\mathscr{F}(w)=\frac{(-1)^{\ell}}{\ell} w^{\ell}$. This means that the power series representation of this function only has one term, and the other coefficients are zero. So $f_{\beta}$ is only nonzero if $\beta=\ell$. So, we have $f_{\beta}=\frac{(-1)^{\beta}}{\ell} \delta[\ell-\beta]$.

\section{Appendix J: Proving the identity in Equation (E26)}

We want to show that

$$
\sum_{\mu} \frac{(-1)^{\mu}}{\mu+1} B_{\mu-1}^{\ell-1} B_{\beta}^{\mu}=\frac{(-1)^{\beta+1}}{\ell(\ell+1)} u(\ell-\beta-1)+\frac{(-1)^{\beta}}{\ell+1} \delta[\ell-\beta]
$$

Let us define $f_{\beta} \stackrel{\text { def }}{=} \sum_{\mu} \frac{(-1)^{\mu}}{\mu+1} B_{\mu-1}^{\ell-1} B_{\beta}^{\mu}$, and $\mathscr{F}(w) \stackrel{\text { def }}{=} \sum_{\beta} f_{\beta} w^{\beta}$. We can find $f(w)$ by interchanging the order of summation as follows:

$$
\mathscr{F}(w)=\sum_{\mu} \sum_{\beta} \frac{(-1)^{\mu}}{\mu+1} B_{\mu-1}^{\ell-1} B_{\beta}^{\mu} w^{\beta}=\sum_{\mu} \frac{(-1)^{\mu}}{\mu+1} B_{\mu-1}^{\ell-1}(1+w)^{\mu}=\sum_{\mu} \frac{[-(1+w)]^{\mu}}{\mu+1} B_{\mu-1}^{\ell-1}
$$

Let us define $z \stackrel{\text { def }}{=}-(1+w)$. Then, we have

$$
\begin{aligned}
\mathscr{F}(w) & =\sum_{\mu} z^{\mu} \mu+1 B_{\mu-1}^{\ell-1}=\sum_{\mu} z^{\mu} \mu+1 B_{\mu-1}^{\ell-1}=z^{-1} \int^{z} z \sum_{\mu} z^{\mu} B_{\mu-1}^{\ell-1} \\
& =z^{-1}\left[\frac{(1+z)^{\ell+1}-1}{\ell+1}-\frac{(1+z)^{\ell}-1}{\ell}\right]=\frac{(-1)^{\ell}}{1+w}\left[\frac{w^{\ell+1}}{\ell+1}+\frac{w^{\ell}}{\ell}-\frac{(-1)^{\ell}}{\ell(\ell+1)}\right]
\end{aligned}
$$


Expanding this results in powers of $w$ is straightforward. The result is

$$
\mathscr{F}(w)=\frac{(-1)^{\ell}}{\ell+1} w^{\ell+1} \sum_{j}(-1)^{j} w^{j}+\frac{(-1)^{\ell}}{\ell} w^{\ell} \sum_{j}(-1)^{j} w^{j}-\frac{1}{\ell+1} w^{\ell(\ell+1)} \sum_{j}(-1)^{j} w^{j}
$$

The overall coefficient of exponents of $w$ that are less than $\beta$ vanishes. We get: $f_{\beta}=\frac{(-1)^{\beta+1}}{\ell(\ell+1)}$ for $\ell>\beta$, and $f_{\beta}=\frac{(-1)^{\beta}}{\ell+1}$ for $\ell=\beta$, which is equivalent to $(\mathrm{J} 1)$.

\section{Appendix K: Proving the identity in Equation (E27)}

We want to show that

$$
\sum_{\mu} \frac{(-1)^{\mu}}{\mu+3} B_{\mu-1}^{\ell-1} B_{\beta}^{\mu}=\frac{(-1)^{\beta+1} 3(\beta+1)(\beta+2)}{\ell(\ell+1)(\ell+2)(\ell+3)} u(\ell-\beta-1)+\frac{(-1)^{\beta}}{(\ell+3)} \delta[\ell-\beta]
$$

Let us define $f_{\beta} \stackrel{\text { def }}{=} \sum_{\mu} \frac{(-1)^{\mu}}{\mu+3} B_{\mu-1}^{\ell-1} B_{\beta}^{\mu}$, and $\mathscr{F}(w) \stackrel{\text { def }}{=} \sum_{\beta} f_{\beta} w^{\beta}$. By interchanging the summation order, we have

$$
\begin{aligned}
\mathscr{F}(w) & =\sum_{\mu} \frac{(-1)^{\mu}}{\mu+3} B_{\mu-1}^{\ell-1} \sum_{\beta} B_{\beta}^{\mu} w^{\beta}=\sum_{\mu} \frac{(-1)^{\mu}}{\mu+3} B_{\mu-1}^{\ell-1}(1+w)^{\mu} \\
& =\sum_{\mu} \frac{[-(1+w)]^{\mu}}{\mu+3} B_{\mu-1}^{\ell-1}
\end{aligned}
$$

Now let us define $z \stackrel{\text { def }}{=}-(1+w)$. Then, we have

$$
\begin{aligned}
\mathscr{F}(w)= & \sum_{\mu} \frac{z^{\mu}}{\mu+3} B_{\mu-1}^{\ell-1}=z^{-3} \\
& \times \sum_{\mu} \frac{z^{\mu+3}}{\mu+3} B_{\mu-1}^{\ell-1}=z^{-3} \int^{z} \sum_{\mu} z^{\mu+2} B_{\mu-1}^{\ell-1}=z^{-3} \int^{z} z^{3}(1+z)^{\ell-1} \\
= & \frac{(z+1)^{\ell}}{z^{3}}\left[\frac{z^{3}}{\ell}-\frac{3 z^{2}(1+z)}{\ell(\ell+1)}+\frac{6 z(1+z)^{2}}{\ell(\ell+1)(\ell+2)}-\frac{6(1+z)^{3}}{\ell(\ell+1)(\ell+2)(\ell+3)}\right] \\
& +\frac{6}{z^{3} \ell(\ell+1)(\ell+2)(\ell+3)}
\end{aligned}
$$

Expressing this in terms of $w$, we get

$$
\begin{aligned}
\mathscr{F}(w)= & \frac{(-w)^{\ell+1}}{(1+w)^{3}}\left[\frac{-(1+w)^{3}}{\ell}+\frac{3(1+w)^{2} w}{\ell(\ell+1)}-\frac{6(1+w) w^{2}}{\ell(\ell+1)(\ell+2)}\right. \\
& \left.+\frac{6 w^{3}}{\ell(\ell+1)(\ell+2)(\ell+3)}\right] \\
& -\frac{6}{(1+w)^{3} \ell(\ell+1)(\ell+2)(\ell+3)}
\end{aligned}
$$

Expanding this in powers of $w$ is straightforward. So, we arrive at $f_{\beta}=\frac{(-1)^{\ell}}{\ell+3}$ for $\beta=\ell$, and $f_{\beta}=\frac{(-1)^{\beta+1} 3(\beta+1)(\beta+2)}{\ell(\ell+1)(\ell+2)(\ell+3)}$ for $\ell>\beta$; hence, the proof is concluded. 


\section{Appendix L: Proving the identity in Equation (E34)}

We want to show that

$$
\sum_{\theta} \frac{(-1)^{\theta}}{k+\theta} B_{\mu+1}^{k+\theta} B_{\theta}^{\mu+1}=\frac{(-1)^{\mu+1}}{(\mu+1)} \delta_{k}
$$

Let us define $h_{k} \stackrel{\text { def }}{=} \sum_{\theta} \frac{(-1)^{\theta}}{k+\theta} B_{\mu+1}^{k+\theta} B_{\theta}^{\mu+1}$, and $\mathscr{H}(x)=\sum_{k} h_{k} x^{k}$. We have

$$
\begin{aligned}
\mathscr{H}(x) & =\sum_{\theta} x^{-\theta}(-1)^{\theta} B_{\theta}^{\mu+1} \sum_{k} B_{\mu+1}^{k+\theta} \frac{x^{k+\theta}}{k+\theta}=\sum_{\theta} x^{-\theta}(-1)^{\theta} B_{\theta}^{\mu+1} \int^{x} \sum_{k} B_{\mu+1}^{k+\theta} x^{k+\theta-1} d x \\
& =\left[\sum_{\theta} x^{-\theta}(-1)^{\theta} B_{\theta}^{\mu+1}\right] \int^{x} x^{-1} \sum_{k} B_{\mu+1}^{k+\theta} x^{k+\theta} d x=\left(\frac{x-1}{x}\right)^{\mu+1} \int^{x} \frac{x^{\mu}}{(1-x)^{\mu+2}}
\end{aligned}
$$

Performing the integration, we have $\int^{x} \frac{x^{\mu}}{(1-x)^{\mu+2}}=\frac{x^{\mu+1}}{(1+\mu)(1-x)^{\mu+1}}$. So, we get

$$
\mathscr{H}(x)=\left(\frac{x-1}{x}\right)^{\mu+1} \frac{x^{\mu+1}}{(1+\mu)(1-x)^{\mu+1}}=\frac{(-1)^{\mu}}{\mu+1}
$$

So, the result does not depend on $x$, which means that there only is a delta function at the origin, multiplying by $x^{0}$ in the power series. We get $h_{k}=\frac{(-1)^{\mu}}{\mu+1} \delta_{k}$, so the proof is completed.

\section{Appendix M: Proving the identity in Equation (E40)}

We want to show that

$$
\sum_{v} B_{1}^{\xi-v} \frac{B_{\mu}^{v}}{v}=\frac{1}{\mu} B_{\mu+1}^{\xi}
$$

Let us define $h(\xi) \stackrel{\text { def }}{=} \sum_{v} B_{1}^{\xi-v} \frac{B_{\mu}^{v}}{v}$, and $\mathscr{H}(x)=\sum_{\xi} h(\xi) x^{\xi}$. We have

$$
\begin{aligned}
\mathscr{H}(x) & =\sum_{v, \xi} B_{1}^{\xi-v} \frac{B_{\mu}^{v}}{v} x^{\xi}=\sum_{v} \frac{B_{\mu}^{v}}{v} x^{v} \sum_{\xi} B_{1}^{\xi-v} x^{\xi-v}=\left[\sum_{v} \frac{B_{\mu}^{v}}{v} x^{v}\right] \frac{x}{(1-x)^{2}} \\
& =\frac{x}{(1-x)^{2}}\left[\int^{x} \sum_{v} B_{\mu}^{v} x^{v-1} d x\right]=\frac{x}{(1-x)^{2}}\left[\int^{x} \frac{1}{x} \sum_{v} B_{\mu}^{v} x^{v} d x\right] \\
& =\frac{x}{(1-x)^{2}} \int^{x} \frac{x^{\mu-1}}{(1-x)^{\mu+1}}=\frac{1}{\mu}\left[\frac{x}{(1-x)^{2}}\right]\left[\frac{x^{\mu}}{(1-x)^{\mu}}\right] \\
& =\frac{1}{\mu}\left[\sum_{\xi} \xi x^{\xi}\right]\left[\sum_{\xi} B_{\mu-1}^{\xi-1}\right]=\sum_{\xi} \frac{1}{\mu}\left[\sum_{\rho} \rho B_{\mu-1}^{\xi-1-\rho}\right] x^{\xi}
\end{aligned}
$$

Now let us find the sum $\pi(\mu) \stackrel{\text { def }}{=} \sum_{\rho} \rho B_{\mu-1}^{\xi-1-\rho}$. Also, define $\pi(x) \stackrel{\text { def }}{=} \sum_{\mu} \pi(\mu) x^{\mu}$. We have

$$
\begin{aligned}
\pi(x) & =\sum_{\rho} \rho x \sum_{\mu} B_{\mu-1}^{\xi-1-\rho} x^{\mu-1}=\sum_{\rho} \rho x(1+x)^{\xi-1-\rho}=x(1+x)^{\xi-2} \sum_{\rho} \rho\left[\frac{1}{1+x}\right]^{\rho-1} \\
& =x(1+x)^{\xi-2} \frac{1}{\left[\left(1-\frac{1}{1+x}\right)\right]^{2}}=\frac{(1+x)^{\xi}}{x}=\sum_{\rho} B_{\xi}^{\mu+1} x^{\mu}
\end{aligned}
$$


So, we have found that $\pi(\mu)=B_{\xi}^{\mu+1}$. Plugging this in the last line of (M2), we get $h(\xi)=\frac{1}{\mu} B_{\xi}^{\mu+1}$. Hence, the proof is complete.

\section{Appendix N: Proving the identity in Equation (E42)}

We want to show that

$$
\sum_{\theta} \frac{(-1)^{\theta}}{k+\theta} B_{\mu+1}^{k+\theta} B_{\theta}^{\mu+2}=\frac{(-1)^{\mu+1}}{(\mu+1)}\left[\delta_{k}-\delta_{k+1}\right]
$$

Every step up to (L2) is identical to those in Appendix L.

$$
\begin{aligned}
\mathscr{H}(x) & =\sum_{\theta} x^{-\theta}(-1)^{\theta} B_{\theta}^{\mu+2} \sum_{k} B_{\mu+1}^{k+\theta} \frac{x^{k+\theta}}{k+\theta}=\sum_{\theta} x^{-\theta}(-1)^{\theta} B_{\theta}^{\mu+2} \int^{x} \sum_{k} B_{\mu+1}^{k+\theta} x^{k+\theta-1} d x \\
& =\left[\sum_{\theta} x^{-\theta}(-1)^{\theta} B_{\theta}^{\mu+2}\right] \int^{x} x^{-1} \sum_{k} B_{\mu+1}^{k+\theta} x^{k+\theta} d x=\left(\frac{x-1}{x}\right)^{\mu+2} \int^{x} \frac{x^{\mu}}{(1-x)^{\mu+2}}
\end{aligned}
$$

The result is

$$
\mathscr{H}(x)=\left(\frac{x-1}{x}\right)^{\mu+2} \frac{x^{\mu+1}}{(1+\mu)(1-x)^{\mu+1}}=\frac{(-1)^{\mu+1}}{\mu+1}\left(1-x^{-1}\right)
$$

which means that $h_{k}=\frac{(-1)^{\mu+1}}{\mu+1}\left[\delta_{k}-\delta_{k+1}\right]$, as desired.

\section{Appendix O: Proving the identity in Equation (E48)}

We want to show that $\sum_{v} B_{3}^{\xi-v} \frac{B_{\mu}^{v}}{v}=\frac{B_{\mu+3}^{\xi}}{\mu}$. Let us define $h(\xi) \stackrel{\text { def }}{=} \sum_{v} B_{3}^{\xi-v} \frac{B_{\mu}^{v}}{v}$, and also the generating function $\mathscr{H}(x) \stackrel{\text { def }}{=} \sum_{\xi} h(\xi) x^{\xi}$. Then, we have

$$
\begin{aligned}
\mathscr{H}(x) & =\sum_{\xi} \sum_{v} x^{v} B_{3}^{\xi-v} \frac{B_{\mu}^{v}}{v} x^{\xi-v}=\sum_{v} x^{v} \frac{B_{\mu}^{v}}{v} \sum_{\xi-v} B_{3}^{\xi-v} x^{\xi-v}=\sum_{v} x^{v} \frac{B_{\mu}^{v}}{v} \frac{x^{3}}{(1-x)^{4}} \\
& =\frac{x^{3}}{(1-x)^{4}} \sum_{v} B_{\mu}^{v} \frac{x^{v}}{v}=\frac{x^{3}}{(1-x)^{4}} \int^{x} \sum_{v} B_{\mu}^{v} x^{v-1} d x=\frac{x^{3}}{(1-x)^{4}} \int^{x} \frac{1}{x} \sum_{v} B_{\mu}^{v} x^{v} d x \\
& =\frac{x^{3}}{(1-x)^{4}} \int^{x} \frac{1}{x} \frac{x^{\mu}}{(1-x)^{\mu+1}} d x=\frac{x^{3}}{(1-x)^{4}} \frac{1}{\mu} \frac{x^{\mu}}{(1-x)^{\mu}}=\frac{1}{\mu} \frac{x^{3+\mu}}{(1-x)^{\mu+4}} \\
& =\sum_{\xi} \underbrace{\frac{1}{\mu} B_{\mu+3}^{\xi}}_{=h(\xi)} x^{\xi}
\end{aligned}
$$

\section{Appendix P: Proving the identity in Equation (E50)}

We want to show that

$$
\sum_{\theta} \frac{(-1)^{\theta}}{k+\theta} B_{\mu+3}^{k+\theta-\beta} B_{\theta}^{\mu+2}=\frac{(-1)^{\mu}}{\mu+3}\left[1-\frac{B_{\beta-1}^{k-1}}{B_{k-\beta}^{\mu+k+2}}\right] u(k-\beta-1)
$$


Let us define $h(k) \stackrel{\text { def }}{=} \sum_{\theta} \frac{(-1)^{\theta}}{k+\theta} B_{\mu+3}^{k+\theta-\beta} B_{\theta}^{\mu+2}$, and $\mathscr{H}(x) \stackrel{\text { def }}{=} \sum_{k} h_{k} x^{k}$. Then we have

$$
\begin{aligned}
\mathscr{H}(x) & =\sum_{\theta}(-1)^{\theta} B_{\theta}^{\mu+2} x^{-\theta} \sum_{k} \frac{x^{k+\theta}}{k+\theta} B_{\mu+3}^{k+\theta-\beta} \\
& =\sum_{\theta}\left(\frac{-1}{x}\right)^{\theta} B_{\theta}^{\mu+2} \int^{x} \sum_{k} B_{\mu+3}^{k+\theta-\beta} x^{k+\theta-1} d x \\
& =\sum_{\theta}\left(\frac{-1}{x}\right)^{\theta} B_{\theta}^{\mu+2} \int^{x} x^{\beta-1} \sum_{k} B_{\mu+3}^{k+\theta-\beta} x^{k+\theta-\beta} d x \\
& =\left[\sum_{\theta}\left(\frac{-1}{x}\right)^{\theta} B_{\theta}^{\mu+2}\right] \int^{x} x^{\beta-1} \frac{x^{\mu+3}}{(1-x)^{\mu+4}} d x \\
& =\left(1-\frac{1}{x}\right)^{\mu+2} \int^{x} \frac{x^{\mu+\beta+2}}{(1-x)^{\mu+4}} d x=\left(\frac{x-1}{x}\right)^{\mu+2} \int^{x} \frac{x^{\mu+\beta+2}}{(1-x)^{\mu+4}} d x
\end{aligned}
$$

Now, the objective is to find the coefficients of $x^{k}$ in the power series expansion of the last term above, in terms of $x$. Let us define $f_{1}(x) \stackrel{\text { def }}{=}\left(\frac{x-1}{x}\right)^{\mu+2}$, and $f_{2}(x) \stackrel{\text { def }}{=}$ $\int^{x} \frac{x^{\mu+\beta+2}}{(1-x)^{\mu+4}} d x$. We have: $\mathscr{H}(x)=\sum_{k} h_{k} x^{k}=f_{1}(x) f_{2}(x)$. Taking the derivative from both sides, we get

$$
\sum_{k}(k+1) h_{k+1} x^{k}=f_{1}^{\prime}(x) f_{2}(x)+f_{1}(x) f_{2}^{\prime}(x)=\frac{f_{1}^{\prime}(x)}{f_{1}(x)} \mathscr{H}(x)+f_{1}(x) f_{2}^{\prime}(x)
$$

It is elementary to show that the following holds: $\frac{f_{1}^{\prime}(x)}{f_{1}(x)}=\frac{-(\mu+2)}{x(1-x)}$. Substituting this into the right-hand side of (P3), and also noting that the derivative in $f_{2}^{\prime}(x)$ eliminates the integration sign, we get $\sum_{k}(k+1) h_{k+1} x^{k}=f_{1}^{\prime}(x) f_{2}(x)+f_{1}(x) f_{2}^{\prime}(x)=$ $\frac{-(\mu+2)}{x(1-x)} \mathscr{H}(x)+\frac{(-1)^{\mu} x^{\beta}}{(1-x)^{2}}$. Expanding the right-hand side in powers of $x$, and then equating the coefficients of each power, gives

$$
\begin{aligned}
& (k+1) h_{k+1}=-(\mu+2) u(k+1) * h_{k}+(k+1-\beta) u(k-\beta) \\
& =-(\mu+2) \sum_{j=-\infty}^{k+1} h_{j}+(-1)^{\mu}(k+1-\beta) u(k-\beta)
\end{aligned}
$$

Writing down the same equation for $k-1$ rather than $k$ gives

$$
k h_{k}=-(\mu+2) \sum_{j=-\infty}^{k} h_{j}+(-1)^{\mu}(k-\beta) u(k-1-\beta)
$$

Subtracting this from (P4), we get $(k+1) h_{k+1}-k h_{k}=-(\mu+2) h_{k+1}+(-1)^{\mu}$. This can be rearranged to give

$$
h_{k+1}=\left[\frac{k}{\mu+k+3}\right] h_{k}+\frac{(-1)^{\mu}}{\mu+k+3}
$$

This is a first-order linear inhomogeneous difference equation, whose formal closedform solution is known (Bender and Orszag, 1978). However, let us write the first few terms and find the solution through speculation. From (P1), it can be readily 
seen that $h_{\beta}=0$. Also, $h_{\beta+1}=\frac{(-1)^{\mu}}{\mu+\beta+3}$. For the next term, we have

$$
h_{\beta+2}=(-1)^{\mu}\left[\frac{\beta+1}{(\mu+\beta+4)(\mu+\beta+3)}\right]+\frac{(-1)^{\mu}}{\mu+\beta+4}=\frac{(-1)^{\mu}}{\mu+3}\left[1-\frac{B_{\beta-1}^{\beta+1}}{B_{2}^{\mu+\beta+4}}\right]
$$

For the next term $h_{\beta+3}$, we have

$$
h_{\beta+3}=\left[\frac{\beta+2}{\mu+\beta+5}\right] \frac{(-1)^{\mu}}{\mu+3}\left[1-\frac{B_{\beta-1}^{\beta+1}}{B_{2}^{\mu+\beta+4}}\right]+\frac{(-1)^{\mu}}{\mu+\beta+5}=\frac{(-1)^{\mu}}{\mu+3}\left[1-\frac{B_{\beta-1}^{\beta+2}}{B_{3}^{\mu+\beta+5}}\right]
$$

We recognize that for $h_{k}$ the general expression is

$$
h_{k}=\frac{(-1)^{\mu}}{\mu+3}\left[1-\frac{B_{\beta-1}^{k-1}}{B_{k-\beta}^{\mu+k+2}}\right] u(k-\beta-1)
$$

The step function exists because for $k \leqslant \beta$, the two binomial factors in the original summand cannot be simultaneously nonzero. That is, the $B_{\theta}^{\mu+2}$ factor imposes $\theta_{\max }=\mu+2$. The $B_{\mu+3}^{k+\theta-\beta}$ factor is nonzero only when $\theta \geqslant \mu+3+\beta-k$. This is synonymous with $\theta_{\min }=\mu+3+\beta-k$. We must have $\theta_{\max } \geqslant \theta_{\min }$ for the original sum to have at least one nonzero term. So, we must have $\mu+2 \geqslant \mu+3+\beta-k$, or equivalently $k \geqslant \beta+1$. Now, we check that according to (P6), the following holds: $(\mu+k+3) h_{k+1}-k h_{k}=(-1)^{\mu}$. Calculating the left-hand side, we get

$$
\begin{aligned}
& (\mu+k+3) \frac{(-1)^{\mu}}{\mu+3}\left[1-\frac{k !}{\frac{(\beta-1) !(k+1-\beta) !}{(\mu+\beta+2) !(k+1-\beta) !}}\right] \\
& -k \frac{(-1)^{\mu}}{\mu+3}\left[1-\frac{\frac{(k-1) !}{(\beta-1) !(k-\beta) !}}{\frac{(\mu+k+2) !}{(\mu+\beta+2) !(k-\beta) !}}\right] \\
& =(\mu+3) \frac{(-1)^{\mu}}{\mu+3}-\frac{k !(\mu+\beta+2) !}{(\beta-1) !(\mu+k+2) !}\left[\frac{(k+1-\beta) !}{(k+1-\beta) !}-\frac{(k-\beta) !}{(k-\beta) !}\right]=(-1)^{\mu}
\end{aligned}
$$

which concludes the proof.

\section{Appendix Q: Proving the identity in Equation (E56)}

We want to show that

$$
\sum_{\theta} \frac{(-1)^{\theta}}{k+\theta} B_{\mu+1}^{k+\theta} B_{\theta}^{\mu}=\frac{(-1)^{\mu+1}}{(\mu+1)}, \forall k
$$

Every step up to (L2) is identical to those in Appendix L:

$$
\begin{aligned}
\mathscr{H}(x) & =\sum_{\theta} x^{-\theta}(-1)^{\theta} B_{\theta}^{\mu} \sum_{k} B_{\mu+1}^{k+\theta} \frac{x^{k+\theta}}{k+\theta}=\sum_{\theta} x^{-\theta}(-1)^{\theta} B_{\theta}^{\mu} \int^{x} \sum_{k} B_{\mu+1}^{k+\theta} x^{k+\theta-1} d x \\
& =\left[\sum_{\theta} x^{-\theta}(-1)^{\theta} B_{\theta}^{\mu}\right] \int^{x} x^{-1} \sum_{k} B_{\mu+1}^{k+\theta} x^{k+\theta} d x=\left(\frac{x-1}{x}\right)^{\mu} \int^{x} \frac{x^{\mu}}{(1-x)^{\mu+2}}
\end{aligned}
$$


The result is

$$
\mathscr{H}(x)=\left(\frac{x-1}{x}\right)^{\mu} \frac{x^{\mu+1}}{(1+\mu)(1-x)^{\mu+1}}=\frac{(-1)^{\mu+1}}{\mu+1}\left(\frac{x}{1-x}\right)=\frac{(-1)^{\mu+1}}{\mu+1} \sum_{k} x^{k}
$$

which means that $h_{k}=\frac{(-1)^{\mu+1}}{\mu+1} \forall k$, as we intended to prove.

\section{Appendix R: Proving the identity in Equation (F2)}

In this appendix, we want to ascertain the validity of an identity that we have used in the evaluation of $Q_{k \ell}(t)+Q_{\ell k}(t)$ in Appendix F. As given in (F2), the identity is

$$
\begin{gathered}
\frac{B_{\beta-1}^{k-1}}{\ell(\ell+1)} \sum_{\mu=\beta}^{\ell}(-1)^{\mu} \frac{B_{\beta}^{\mu} B_{\mu+1}^{\ell+1}}{B_{k-\beta}^{\mu+k+2}}+\frac{B_{\beta-1}^{\ell-1}}{k(k+1)} \sum_{\mu=\beta}^{k}(-1)^{\mu} \frac{B_{\beta}^{\mu} B_{\mu+1}^{k+1}}{B_{\ell-\beta}^{\mu+\ell+2}} \\
=\beta(\beta+1)(-1)^{\beta} \frac{(k+2) B_{\ell}^{k+\ell+2}+(\ell+1) B_{\beta+1}^{2 \beta+2} B_{k-\beta}^{k+\ell-2 \beta}}{k(k+1)(k+2) \ell(\ell+1) B_{\ell}^{k+\ell+2}}
\end{gathered}
$$

We prove this by finding the generating function of both sides of (R1), and showing that the generating functions are identical. Since the inversion is one to one, this will conclude the proof.

First, we simplify the left-hand side of (R1). We have

$$
\begin{aligned}
(-1)^{\mu} \frac{B_{\beta-1}^{k-1}}{\ell(\ell+1)} \frac{B_{\beta}^{\mu} B_{\mu+1}^{\ell+1}}{B_{k-\beta}^{\mu+k+2}} & (-1)^{\mu} \frac{(k-1) !}{(\beta-1) !(k-\beta) ! \ell(\ell+1)} \frac{\mu !(\ell+1) !(k-\beta) !(\mu+\beta+2) !}{\beta !(\mu-\beta) !(\mu+1) !(\ell-\mu) !(\mu+k+2) !} \\
= & (-1)^{\mu} \frac{(k+1) !(\ell+1) !}{(\beta-1) ! \beta ! k(k+1) \ell(\ell+1)(\mu+1)} \frac{(\mu+\beta+2) !}{(\mu-\beta) !(\ell-\mu) !(\mu+k+2) !} \\
& \times \frac{(2 \beta+2) !(k+\ell+2) !}{(2 \beta+2) !(k+\ell+2) !} \\
= & (-1)^{\mu} \frac{(2 \beta+2) ! B_{2 \beta+2}^{\mu+\beta+2} B_{\mu+k+2}^{\ell+k+2}}{k(k+1) \ell(\ell+1)(\beta-1) ! \beta !(\mu+1) B_{k+1}^{k+\ell+2}} \\
= & (-1)^{\mu} \frac{\beta(\beta+1)^{2} B_{\beta+1}^{2 \beta+2}}{k(k+1) \ell(\ell+1) B_{k+1}^{k+\ell+2}} \frac{B_{2 \beta+2}^{\mu+\beta+2} B_{\mu+k+2}^{\ell+k+2}}{\mu+1}
\end{aligned}
$$

Inserting this into the left-hand side of (R1), multiplying both sides by $k(k+1) \ell(\ell+1)$, and dividing both sides by $\beta(\beta+1)$, we arrive at the following equivalent expression:

$$
\begin{aligned}
& \frac{(\beta+1) B_{\beta+1}^{2 \beta+2}}{B_{\ell+1}^{k+\ell+2}}\left[\sum_{\mu=\beta}^{\ell}(-1)^{\mu} \frac{B_{2 \beta+2}^{\mu+\beta+2} B_{\mu+k+2}^{\ell+k+2}}{\mu+1}+\sum_{\mu=\beta}^{k}(-1)^{\mu} \frac{B_{2 \beta+2}^{\mu+\beta+2} B_{\mu+\ell+2}^{\ell+k+2}}{\mu+1}\right] \\
& =(-1)^{\beta} \frac{(k+2) B_{\ell}^{k+\ell+2}+(\ell+1) B_{\beta+1}^{2 \beta+2} B_{k-\beta}^{k+\ell-2 \beta}}{(k+2) B_{\ell}^{k+\ell+2}}
\end{aligned}
$$


Now, using the fact that $B_{\ell+1}^{k+\ell+2}=\frac{k+2}{\ell+1} B_{\ell}^{k+\ell+2}$, this can be transformed into

$$
\begin{aligned}
& \sum_{\mu=\beta}^{\ell}(-1)^{\mu} \frac{B_{2 \beta+2}^{\mu+\beta+2} B_{\mu+k+2}^{\ell+k+2}}{\mu+1}+\sum_{\mu=\beta}^{k}(-1)^{\mu} \frac{B_{2 \beta+2}^{\mu+\beta+2} B_{\mu+\ell+2}^{\ell+k+2}}{\mu+1} \\
& \quad=(-1)^{\beta} \frac{B_{\ell+1}^{k+\ell+2}+B_{\beta+1}^{2 \beta+2} B_{k-\beta}^{k+\ell-2 \beta}}{(\beta+1) B_{\beta+1}^{2 \beta+2}}
\end{aligned}
$$

Since (R4) and (R1) are equivalent, we will prove the validity of (R4). Then, the validity of (R1) will follow. To proceed, we show that the generation functions of the two sides of (R4) are identical. Since the inversion is one to one, it will be synonymous with the equality of two sides of (R4). Note that the upper bounds of both sums in (R1) can be replaced by infinity, because the bounds are automatically imposed by the binomial coefficients in the summands. Similarly, the lower bounds can be set to $-\infty$. Thus, the summation bounds are redundant and will be skipped hereafter. In the next steps, we use the following elementary identity:

$$
\sum_{j} B_{i}^{j} x^{j}=\frac{x^{i}}{(1-x)^{i+1}}
$$

This readily follows by Taylor expanding the right-hand side. Let us multiply both sides of (R4) by $y^{\ell}$ and sum over all values of $\ell$. Let us denote the generating functions of the left- and right-hand sides by $H_{L}$ and $H_{R}$, respectively. The goal is to prove that $H_{L}$ and $H_{R}$ are identical. For the left-hand side, we will have

$$
\begin{aligned}
H_{L} \stackrel{\text { def }}{=} & \sum_{\mu} \sum_{\ell}(-1)^{\mu} \frac{B_{2 \beta+2}^{\mu+\beta+2} B_{\mu+k+2}^{\ell+k+2}}{\mu+1} y^{\ell}+\sum_{\mu} \sum_{\ell}(-1)^{\mu} \frac{B_{2 \beta+2}^{\mu+\beta+2} B_{\mu+\ell+2}^{\ell+k+2}}{\mu+1} y^{\ell} \\
= & \sum_{\mu}(-1)^{\mu} \frac{B_{2 \beta+2}^{\mu+\beta+2}}{\mu+1} \sum_{\ell} B_{\mu+k+2}^{\ell+k+2} y^{\ell}+\sum_{\mu}(-1)^{\mu} \frac{B_{2 \beta+2}^{\mu+\beta+2}}{\mu+1} \sum_{\ell} B_{\mu+\ell+2}^{\ell+k+2} y^{\ell} \\
= & \sum_{\mu}(-1)^{\mu} \frac{B_{2 \beta+2}^{\mu+\beta+2}}{\mu+1} y^{-k-2} \sum_{\ell} B_{\mu+k+2}^{\ell+k+2} y^{k+\ell+2} \\
& +\sum_{\mu}(-1)^{\mu} \frac{B_{2 \beta+2}^{\mu+\beta+2}}{\mu+1} y^{-k-2} \sum_{\ell} B_{k-\mu}^{\ell+k+2} y^{k+\ell+2} \\
= & \sum_{\mu}(-1)^{\mu} \frac{B_{2 \beta+2}^{\mu+\beta+2}}{\mu+1} y^{-k-2} \sum_{\theta} B_{\mu+k+2}^{\theta} y^{\theta}+\sum_{\mu}(-1)^{\mu} \frac{B_{2 \beta+2}^{\mu+\beta+2}}{\mu+1} y^{-k-2} \sum_{\theta} B_{k-\mu}^{\theta} y^{\theta} \\
= & \sum_{\mu}(-1)^{\mu} \frac{B_{2 \beta+2}^{\mu+\beta+2}}{\mu+1} y^{-k-2} \frac{y^{\mu+k+2}}{(1-y)^{\mu+k+3}}+\sum_{\mu}(-1)^{\mu} \frac{B_{2 \beta+2}^{\mu+\beta+2}}{\mu+1} y^{-k-2} \frac{y^{k-\mu}}{(1-y)^{k-\mu+1}}
\end{aligned}
$$


where in the last step we have used (R5). Now, we undertake the following rearranging steps:

$$
\begin{aligned}
H_{L}= & \sum_{\mu}(-1)^{\mu} \frac{B_{2 \beta+2}^{\mu+\beta+2}}{\mu+1} y^{-k-2} \frac{y^{\mu+k+2}}{(1-y)^{\mu+k+3}}+\sum_{\mu}(-1)^{\mu} \frac{B_{2 \beta+2}^{\mu+\beta+2}}{\mu+1} y^{-k-2} \frac{y^{k-\mu}}{(1-y)^{k-\mu+1}} \\
= & \frac{1}{(1-y)^{k}}\left[\sum_{\mu}(-1)^{\mu} \frac{B_{2 \beta+2}^{\mu+\beta+2}}{\mu+1} \frac{y^{\mu}}{(1-y)^{\mu+3}}+\sum_{\mu}(-1)^{\mu} \frac{B_{2 \beta+2}^{\mu+\beta+2}}{\mu+1} \frac{y^{-\mu-2}}{(1-y)^{-\mu+1}}\right] \\
= & \frac{1}{(1-y)^{k}}\left[-\sum_{\mu}(-1)^{\mu+1} \frac{B_{2 \beta+2}^{\mu+\beta+2}}{\mu+1} \frac{y^{-1}}{(1-y)^{2}}\left(\frac{y}{1-y}\right)^{\mu+1}\right. \\
& \left.-\sum_{\mu}(-1)^{\mu+1} \frac{B_{2 \beta+2}^{\mu+\beta+2}}{\mu+1} \frac{y^{-1}}{(1-y)^{2}}\left(\frac{y}{1-y}\right)^{-\mu-1}\right]
\end{aligned}
$$

Let us denote $\frac{-y}{1-y}$ by $x$ and denote $\frac{1}{x}$ by $w$. Then, we have

$$
\begin{aligned}
H_{L}= & \frac{y^{-1}}{(1-y)^{k+2}}\left[-\sum_{\mu} B_{2 \beta+2}^{\mu+\beta+2} \frac{x^{\mu+1}}{\mu+1}-\sum_{\mu} B_{2 \beta+2}^{\mu+\beta+2} \frac{w^{\mu+1}}{\mu+1}\right] \\
= & \frac{y^{-1}}{(1-y)^{k+2}}\left[-\int_{0}^{x} \sum_{\mu} B_{2 \beta+2}^{\mu+\beta+2} v^{\mu} d v-\int_{0}^{w} \sum_{\mu} B_{2 \beta+2}^{\mu+\beta+2} v^{\mu} d v\right] \\
= & \frac{y^{-1}}{(1-y)^{k+2}}\left[-\int_{0}^{x} v^{-\beta-2} \sum_{\mu} B_{2 \beta+2}^{\mu+\beta+2} v^{\mu+\beta+2} d v\right. \\
& \left.-\int_{0}^{w} v^{-\beta-2} \sum_{\mu} B_{2 \beta+2}^{\mu+\beta+2} v^{\mu+\beta+2} d v\right] \\
= & \frac{y^{-1}}{(1-y)^{k+2}}\left[-\int_{0}^{x} v^{-\beta-2} \sum_{\theta} B_{2 \beta+2}^{\theta} v^{\theta} d v-\int_{0}^{w} v^{-\beta-2} \sum_{\mu} B_{2 \beta+2}^{\theta} v^{\theta} d v\right] \\
= & \frac{y^{-1}}{(1-y)^{k+2}}\left[-\int_{0}^{x} v^{-\beta-2} \frac{v^{2 \beta+2}}{(1-v)^{2 \beta+3}} d v-\int_{0}^{w} v^{-\beta-2} \frac{v^{2 \beta+2}}{(1-v)^{2 \beta+3}} d v\right]
\end{aligned}
$$

where in the last step again we have used the identity given in (R5). We can simplify the integrands further: $H_{L}=\frac{y^{-1}}{(1-y)^{k+2}}\left[-\int_{0}^{x} \frac{v^{\beta}}{(1-v)^{2 \beta+3}} d x-\int_{0}^{w} \frac{v^{\beta}}{(1-v)^{2 \beta+3}} d v\right]$. It can be easily verified by taking the derivative that the result of the integral is

$$
\int_{0}^{x} \frac{x^{\beta}}{(1-x)^{2 \beta+3}} d x=\frac{-(-1)^{\beta}}{(\beta+1) B_{\beta+1}^{2 \beta+2}} \frac{1}{(x-1)^{2 \beta+2}} \sum_{j=\beta+1}^{2 \beta+2} B_{j}^{2 \beta+2}(-x)^{j}
$$

The sum on the right-hand side is the last $\beta+2$ terms of the expansion of $(1-x)^{2 \beta+2}$. Using this result and replacing $x$ by its definition, which is $\frac{-y}{1-y}$, we have

$$
\begin{aligned}
H_{L}= & \frac{y^{-1}}{(1-y)^{k+2}} \frac{(-1)^{\beta}}{(\beta+1) B_{\beta+1}^{2 \beta+2}} \\
& \times\left[\sum_{j=\beta+1}^{2 \beta+2} B_{j}^{2 \beta+2} y^{j}(1-y)^{2 \beta+2-j}+\sum_{j=\beta+1}^{2 \beta+2} B_{j}^{2 \beta+2}(1-y)^{j} y^{2 \beta+2-j}\right]
\end{aligned}
$$


Looking at the two sums, it can be easily seen that if one expands $[(1-y)+y]^{2 \beta+2}$, which would have $2 \beta+3$ terms, then the last $\beta+2$ terms are given by the first sum and the last $\beta+2$ of them are given by the second sum. So, the middle term, which has the coefficient $B_{\beta+1}^{2 \beta+2}$, has been repeated twice. So, instead of the two sums, we can write 1 , which is the result of $[(1-y)+y]^{2 \beta+2}$, plus the degenerate term, which is $B_{\beta+1}^{2 \beta+2} y^{\beta+1}(1-y)^{\beta+1}$. So, we obtain

$$
H_{L}=\frac{y^{-1}}{(1-y)^{k+2}} \frac{(-1)^{\beta}}{(\beta+1) B_{\beta+1}^{2 \beta+2}}\left[1+B_{\beta+1}^{2 \beta+2} y^{\beta+1}(1-y)^{\beta+1}\right]
$$

Now, we return to (R4) and take the generating function of the right-hand side. We have

$$
\begin{aligned}
H_{R} & \stackrel{\text { def }}{=} \sum_{\ell=0}^{\infty}(-1)^{\beta} \frac{B_{\ell+1}^{k+\ell+2}+B_{\beta+1}^{2 \beta+2} B_{k-\beta}^{k+\ell-2 \beta}}{(\beta+1) B_{\beta+1}^{2 \beta+2}} y^{\ell} \\
& =\frac{(-1)^{\beta}}{\beta+1}\left[\frac{y^{-k-2}}{B_{\beta+1}^{2 \beta+2}} \sum_{\ell=0}^{\infty} B_{k+1}^{k+\ell+2} y^{k+\ell+2}+y^{2 \beta-k} \sum_{\ell=0}^{\infty} B_{k-\beta}^{k+\ell-2 \beta} y^{k+\ell-2 \beta}\right] \\
& =\frac{(-1)^{\beta}}{\beta+1}\left[\frac{y^{-k-2}}{B_{\beta+1}^{2 \beta+2}} \sum_{\theta} B_{k+1}^{\theta} y^{\theta}+y^{2 \beta-k} \sum_{\theta} B_{k-\beta}^{\theta} y^{\theta}\right]
\end{aligned}
$$

Now, in both sums, we can use (R5) to obtain

$$
\begin{aligned}
H_{R} & =\frac{(-1)^{\beta}}{\beta+1}\left[\frac{y^{-k-2}}{B_{\beta+1}^{2 \beta+2}} \sum_{\theta} B_{k+1}^{\theta} y^{\theta}+y^{2 \beta-k} \sum_{\theta} B_{k-\beta}^{\theta} y^{\theta}\right] \\
& =\frac{(-1)^{\beta}}{\beta+1}\left[\frac{y^{-k-2}}{B_{\beta+1}^{2 \beta+2}} \frac{y^{k+1}}{(1-y)^{k+2}}+y^{2 \beta-k} \frac{y^{k-\beta}}{(1-y)^{k-\beta+1}}\right] \\
& =\frac{(-1)^{\beta}}{\beta+1}\left[\frac{1}{B_{\beta+1}^{2 \beta+2}} \frac{y^{-1}}{(1-y)^{k+2}}+\frac{y^{\beta}}{(1-y)^{k-\beta+1}}\right] \\
& =\frac{(-1)^{\beta}}{\beta+1} \frac{y^{-1}}{(1-y)^{k+2}}\left[\frac{1}{B_{\beta+1}^{2 \beta+2}}+y^{\beta+1}(1-y)^{\beta+1}\right] \\
& =\frac{(-1)^{\beta}}{\beta+1} \frac{y^{-1}}{(1-y)^{k+2}} \frac{\left[1+B_{\beta+1}^{2 \beta+2} y^{\beta+1}(1-y)^{\beta+1}\right]}{B_{\beta+1}^{2 \beta+2}}
\end{aligned}
$$

Since (R13) and (R11) are identical, the proof is concluded. 